$\frac{4}{4}$

VelioMI

www.neuromi.it

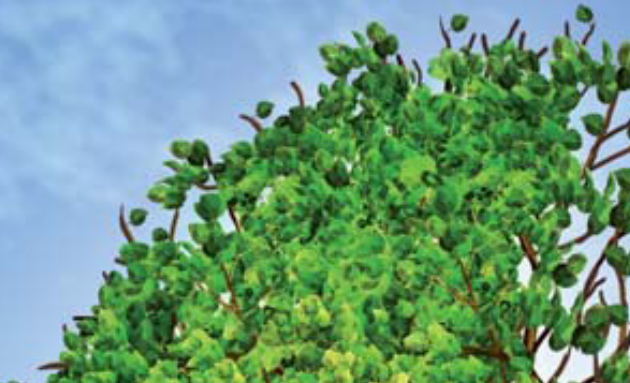

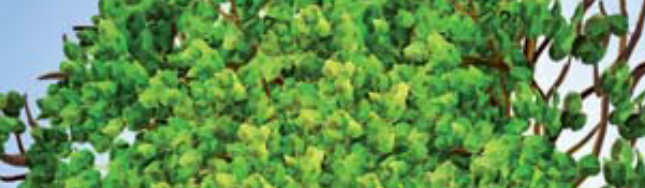

of

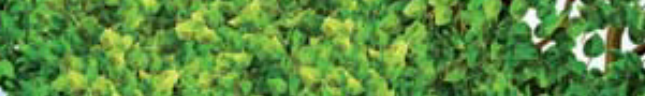

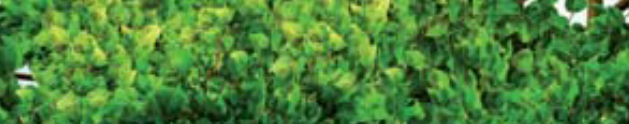

if

Q3.

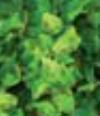

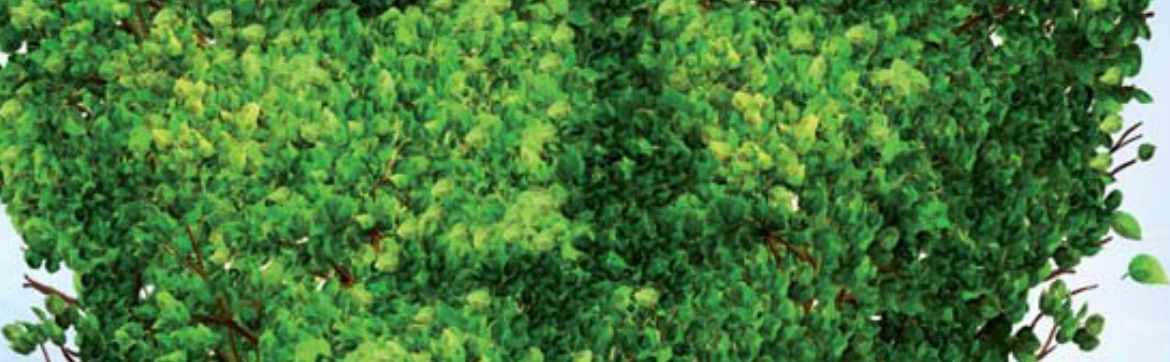

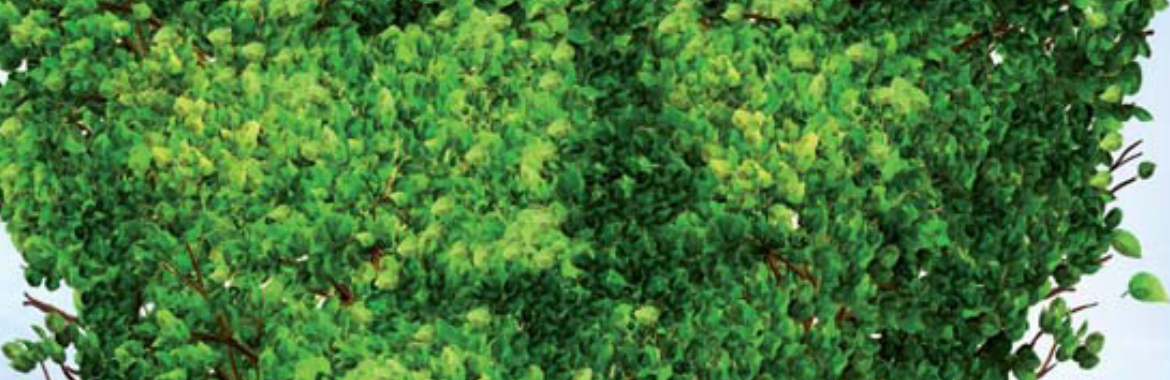

3.

को

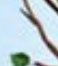

t)

t.

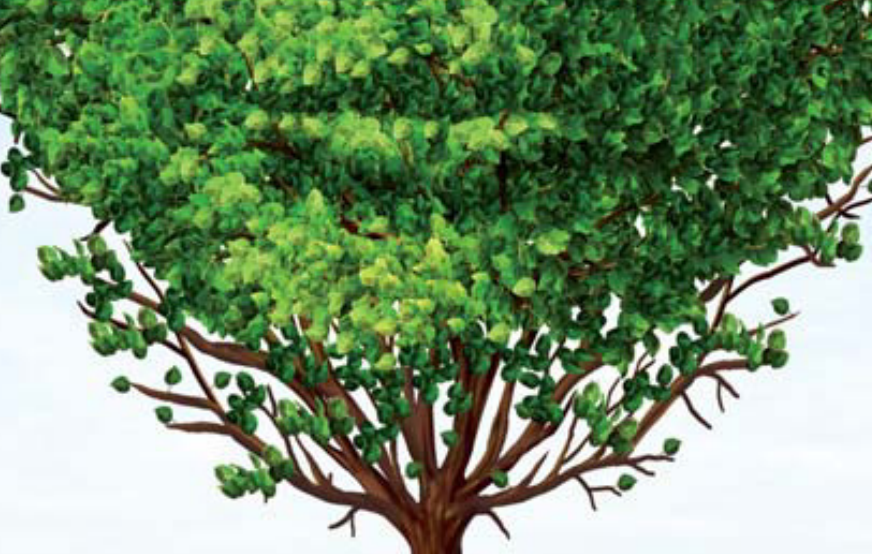

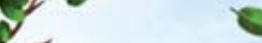

SECOND INTERNATIONAL

-

MEETING OF THE

Milan Center

FOR NEUROSCIENCE

(NEUROMI)

9

PREDICTION

AND PREVENTION

OF DEMENTIA:

NEW HOPE

Milan, July 6-8, 2016

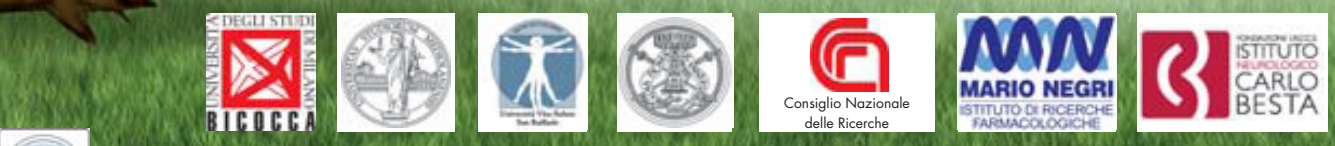

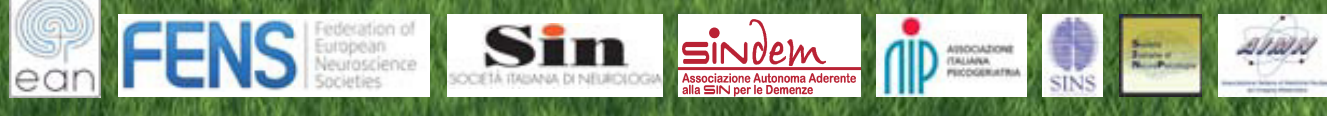




\section{CONGRESS VENUE}

University of Milano-Bicocca

Aula Magna, Building U6,

Piazza del Nuovo Ateneo, 1, Milan

\section{PRESIDENT OF THE CONGRESS}

Carlo Ferrarese

\section{SCIENTIFIC BOARD}

Giorgio Annoni

Ildebrando Appollonio

Gabriella Bottini

Stefano Cappa

Giancarlo Comi

Gianluigi Forloni

Monica Di Luca

Claudio Mariani

Massimo Masserini

Rosa Maria Moresco

Massimo Musicco

Eraldo Paulesu

Daniela Perani

Elio Scarpini

Fabrizio Tagliavini

EXECUTIVE COMMITEE

Antonino Gennaro

Valeria Isella

Fabrizio Piazza

Lucio Tremolizzo

Chiara Zoia

\section{ORGANIZING SECRETARIAT}

Studio ConventurSiena

Via del Cavallerizzo, 1 - 53100 Siena

Tel. 0577270870 / Fax 0577289334

info@neuro.it 
Journal of Alzheimer's Disease

Abstracts for the Second International Meeting of the Milan Center for Neuroscience (Neuromi): Prediction and Prevention of Dementia:

New Hope (Milan, July 6-8, 2016)

\section{CONTENTS}

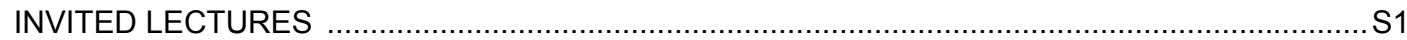

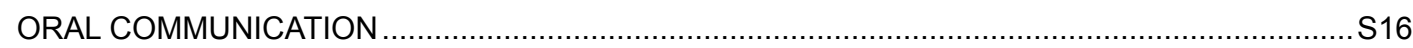

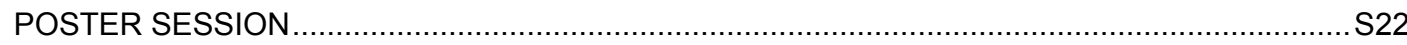

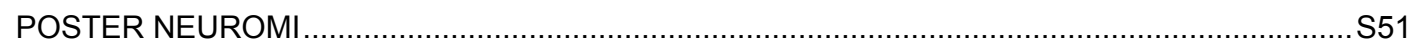

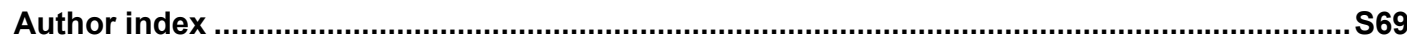





\section{INVITED LECTURES}

\section{Preserved cognition and Alzheimer pathology in the oldest old}

\section{O. Bugiani}

\section{Istituto Neurologico Carlo Besta Milano, Fondazione Golgi-Cenci (Abbiategrasso-MI)}

People aged over 85, referred to as the oldest old, have been increasing in number faster than any other age group in the population and are expected to triplicate by the end of the century. Luckily, they may feel themselves better than most younger old can do, and even be reasonably autonomous, if not healthy at all. Accordingly, some of them could hold and delay major illnesses, among which the dementia. To focus on the latter and its ageing-related prevalence, it is likely, although debated, that it affects the oldest old less than expected compared to the younger elderly (reviews in Perls 2004, Yang et al 2013), and that the association between dementia and plaques and tangles, the most representative lesions of both normal and pathologic brain ageing and so the most commonly investigated, is stronger in the younger elderly than in the older old (Haroutunian et al 2008, Savva et al 2009, Corrada et al 2012). Longevityenabling genes, increased adaptation, and lower risk factors for dementia in mid-life have been proposed as an explanation. Interestingly, these data are in agreement with a meta-analysis of prevalence of dementia which showed that, compared with the ageing-based exponential model, logistic models indicated that prevalence rates were higher from age 75 to 95 years and subsequently lower, so originating a plateau after age 95 and suggesting that dementia is related to one age range rather than to ageing (Ritchie \& Kildea 1995). Discontinuity in the prevalence of dementia should point to vulnerability of allocortical and neocortical neurons modulated by ontogenetic factors that make them variably sensitive to degeneration, and so mark one difference among individuals (Bugiani 2011). Accordingly, the age-related dementia might be explained in terms of neurons more sensitive to degeneration that survive in the brain after inadequate cell selection during development (Arendt 2005). This theory should apply to Alzheimer pathology due to the crucial role of APP, Abeta and related proteins in brain develop- ment. Conversely, privileges of the oldest old might be related to successful cell selection that allows cognitive reserve, neural compensation, and adult neurogenesis to maximise their effects.

\section{Suggestions from epidemiology and clinical epidemiology for prevention and better knowledge of dementia}

\section{Musicco \\ Institute of Biomedical Technologies (Segrate- MI)}

Population studies on occurrence of neurodegenerative dementias show that in Europe the incidence of these disorders is decreasing over time, despite their progressive increasing prevalence. Actually the number of persons with dementia increases in parallel with the progressive aging of our population, but the number of persons who become demented in the different age strata is decreasing. The reasons of this epochal phenomenon are not completely known but may be searched for in the changing pattern of social characteristics, life habits and access to medical interventions of more recent birth cohorts of population. Better education, healthier life styles and more intense control of disease risk factors characterize contemporary elderly populations with respect to those of the past. This trend of incidence reduction is spontaneous and can be considered an unintended effect of the progressive general improving of the social and health conditions of the European population. It remains to be defined, for the future, what predefined health policies should be implemented with the purpose of controlling and preventing neurodegenerative disorders with clear and evidence based interventions. The results of recent preventive interventions on populations at risk of cognitive decline are more than encouraging for designing public health initiatives targeted to fight against neurodegeneration and dementia. In the field of better understanding the relationship between neurodegeneration and aging there are a number of evidence that dementia in the oldest old presents peculiarities that should be taken into account for future interventions and actions. First neurodegeneration seems to compete with cancer in the complex phenomenon of 
senescence since a number of studies reported that the occurrence of cancer is less than expected in persons with neurodegenerative disorders and viceversa. Second, many studies are reporting in the oldest old that many dementias with an Alzheimer phenotype are not sustained by the traditional neuropathological picture of the contemporary presence and amyloid plaques deposition and neuro- phibrillary degeneration. This observations poses the need of reconsidering, at least for the oldest old, our current paradigms about the pathophysiology of dementias.

\section{Ethical Issues on Dementia}

\section{A. Sironi \\ Director of the Research Center on the History of Biomedical Thought, University of Milano Bicocca (Milano)}

Dementia today is an existential condition which leads, through worsening clinical manifestations, to the inexorable exit from the human through the progressive loss of the possibility of establishing cognitive relations until the complete disintegration of the individual personality. In the medical field, in the early and middle stages of the disease the fundamental task is to prevent possible complications, addressing the behavioural changes and treating the psychological symptoms, while in the terminal stages must lead the patient towards the end of his earthly existence in the most dignified way possible. It must be remembered that in the "therapeutic relationship" with the patient and his family, he will always be considered - although demented - as a person worthy of attention and respect. The communication of the diagnosis to the patient - who has the right to know - is the first ethical knot: we must establish what the subject is able to combine the "right to truth" (diagnostic) with the "right to hope" (existential). A second important aspect concerns the consent to medical procedures, which should be viewed in the context of the patient's autonomy and decisional aspects of personal identity and the degree of awareness of the subject. This perspective also raises the issue of "Advance treatment statements" relating to future therapy (use of pain medications, access to palliative care, artificial feeding) and a possible "living will" consistent with the system of values and with the beliefs that the patient expressed in the course of its existence. Finally, a fundamental ethical issue concerns the problems of clinical trials on people incapable of expressing a full consensus. Is important that this problem is addressed in a way that it cannot only provide adequate protection in these subjects, but also so that it can be favoured their participation given that testing could result in real benefits to their health.

\section{Synaptopathology of dementia}

\section{E. Marcello, M. Di Luca \\ Department of Pharmacological and Biomolecular Sciences (Milano)}

Background: Alzheimer's disease (AD) is the most prevalent and socially disruptive illness of aging populations. Genetic and pathological evidence strongly supports the amyloid cascade hypothesis, which states that Amyloid $\beta(\mathrm{A} \beta)$ has an early and crucial role in $\mathrm{AD}$. $\mathrm{A} \beta$ is liberated from the amyloid precursor protein (APP) by BACE and $\gamma$-secretase activity. Alternatively, APP is cleaved within the A $\beta$ domain by ADAM10, which prevents $A \beta$ formation. In addition to $A \beta$, synapse loss has a central role in $\mathrm{AD}$ pathogenesis, rather than just a consequence of cell death. Synapse loss represents an early insult that advances with disease and dendritic spine loss is seen in several AD models.

Aim: We aim at studying the mechanisms underlying the crosstalk between $A \beta$ and synaptic function and failure.

Results: We provide new evidences on local trafficking pathways relevant for $\mathrm{AD}$ pathogenesis. We described the mechanisms responsible for ADAM10 forward trafficking and endocytosis, that are important for $A \beta$ production and spine remodelling. We provided a mechanism according to which ADAM10 local trafficking from dendritic Golgi outposts to the postsynaptic density is mediated by SAP97 and is under the control of PKC-dependent phosphorylation of SAP97. On the other hand, activity-regulated association of ADAM10 with the clathrin adaptor AP2 complex mediates ADAM10 endocytosis. Moreover, ADAM10 local trafficking mechanisms are impaired in $\mathrm{AD}$ patients at the earlier $\mathrm{AD}$ stages.

Conclusions: Taken together these results provide a complete picture of the network of interacting pathways leading to $\mathrm{AD}$ that is mandatory for the development of successful therapies. 


\section{Lymphatic drainage of the brain and the pathology of cerebral amyloid angiopathy}

\author{
M. MacGregor-Sharp ${ }^{1}$, N. Albargothy ${ }^{1}$, A. Morris ${ }^{1}$, \\ R.O. Weller ${ }^{1}$, A. Verma ${ }^{2}$, R.O. Carare ${ }^{1}$ \\ ${ }^{1}$ University of Southampton, Faculty of Medicine, \\ MP806, South Academic Block, Southampton \\ General Hospital (Southampton- UK) \\ ${ }^{2}$ Biogen, 14 Cambridge Center (Cambridge-USA)
}

The brain is an immune privileged organ due to the presence of a blood-brain barrier and the lack of lymphatic vessels. However, it has been clear for several decades that CNS antigens drain into regional lymph nodes. In humans, soluble amyloid $\beta$ is produced by neurons and is deposited in the walls of ageing cerebral blood vessels as cerebral amyloid angiopathy (CAA), providing thus a natural tracer for the drainage of solutes. Our anatomical experimental studies using wild-type mice demonstrate that injection of soluble tracers in the gray matter of the brain results in diffusion and drainage of the tracers along the basement membranes of capillaries and the basement membranes surrounding smooth muscle cells of arteries, towards the surface of the brain. This pathway is effectively the lymphatic drainage pathway of the brain and is restricted to solutes, as nanoparticles of $15 \mathrm{~nm}$ cannot enter the cerebrovascular basement membranes. Ageing, possession of apolipoprotein E4 and vascular immune complexes block this drainage pathway, resulting in CAA. The cerebrovascular basement membranes are also the pathway for convective influx of cerebrospinal fluid (CSF) into the brain. Specifically, the glial-pial basement membranes are the pathways for convective influx of CSF. Using optimally preserved canine brain tissue we have demonstrated that the structure of the wall of arteries is different in gray matter compared to white matter, providing thus a platform for better interpretation of age-related white matter hyperintensities. Our results are significant for the pathology of CAA and for therapeutic delivery of drugs into the brain.

\section{Microglia, neuroinflammation and Alzheimer disease}

\author{
M. T. Heneka, \\ Clinical Neuroscience, Dept. Of Neurology, \\ University of Bonn (Bonn-D)
}

Generation of neurotoxic amyloid- $\beta$ peptides and their deposition along with neurofibrillary tangle formation represent key pathological hallmarks in Alzheimer's disease (AD). Recent evidence suggests that inflammation may be a third important component, which, once initiated in response to neurodegeneration or dysfunction actively contributes to disease progression and chronicity. Microglia is being activated by binding of aggregated proteins or aberrant nucleic acids to pattern recognition receptors, which elicit an innate immune response. The latter is characterized by the release of inflammatory mediators including complement activators and inhibitors, chemokines, cytokines, radical oxygen species and enzyme systems. Exogenous as well as endogenous factors may promote and facilitate neuroinflammation in the AD brain. Thus, degeneration of aminergic brain stem nuclei including the locus ceruleus and the nucleus basalis of Meynert may drive inflammation in their projection areas given the antiinflammatory and neuroprotective action of their key transmitters norepinephrine and acetylcholine. Inflammation may not just occur secondary to degeneration, but actively drive amyloid beta aggregation and APP processing. Modulation of the microglia driven innate immune response at key signalling steps may provide protection. Therefore, antiinflammatory treatment strategies should be considered. Data on microglial activation in AD along with suggestions to modify and alter the pro- into an antiinflammatory phenotype will be reviewed and discussed.

\section{Central and peripheral markers of neuroinflammation: focus on brain Imaging}

R. M. Moresco

IBFM-CNR, CIS San Raffaele Scientific Institute and Center for Neurosciences University of Milan Bicocca (Milano)

The $18 \mathrm{k}$-Da TSPO receptor is part of a multimeric complex whose levels rise in different brain disorders, reflecting macrophage invasion, glial proliferation or both. For this reason, the TSPO antagonist, [11C]PK11195 has been extensively used to image immune system activation during brain infection, inflammation or neuronal injury. The low in vivo signal to noise of [11C]PK11195 -PET images has stimulate the developments of several new 
chemical class of TSPO radiopharmaceuticals. However, binding of new class of radiopharmaceuticals to TSPO is sensitive to Single nucleotide polymorphism at Rs6971 in human beings increasing data variability. In addition not all reactive microglia/macrophage cells express TSPO receptors, and that their level of expression in different cells are heterogeneous. Finally, microglial cells of TSPO $\mathrm{KO}$ animals displayed an impaired mitochondrial metabolism and reduced ATP production. These observation, together with some successful results from TSPO agents used as therapeutics raise the question of the role of this TSPO in immune system and the real meaning of TSPO related images. Despite the low specificity of this target and the large amount of questions remained unsolved, this target represent a unique tool to detect the activation of immune system in human brain. To bypass TSPO limits, several effort of radiochemistry and radiopharmacology are actually focused on the discovery of new ligand selective for specific immune cells phenotype. Radioligand for reactive gliosis ([ $\left.{ }^{11} \mathrm{C}\right]$ Deprenyl) or other microglial targets are under current clinical or preclinical evaluation.

\section{Peripheral markers of neuroinflammation}

\section{Tremolizzo, E. Conti, C. Ferrarese \\ School of Medicine and NeuroMI, University of Milano-Bicocca (Milano)}

Neuroinflammation is a process postulated to be confined to the CNS and to contribute to disease pathogenesis in Alzheimer's disease (AD), among other neurodegenerative disorders. Albeit microglia cells specifically sustain this process, peripheral monocytes significantly contribute transforming in blood-born macrophages, passing through the bloodbrain barrier attracted by increased beta-amyloid (Abeta) production. This interplay between CNS and periphery is further strengthened by the demonstration of the immune reflex, a cholinergic pathway projecting on alpha7- nicotinic receptors expressed by spleen monocytes and blocking the activation of the inflammosome. In this brief talk we will present the conceptual framework for a specific peripheral contribution to the spreading of $\mathrm{AD}$ pathogenic mechanisms, focusing on the role of monocytes. Particular reference will be made to the role of two proteins: TSPO- $18 \mathrm{kDa}$ in chemotaxis and TREM2 in phagocytosis, showing our preliminary ex vivo results in $\mathrm{AD}$ patients.

\section{Do animal models recapitulate Alzheimer's disease?}

\author{
G. Forloni, C. Balducci \\ Department of Neuroscience, IRCCS Istituto di \\ Ricerche Farmacologiche Mario Negri (Milano)
}

Alzheimer's disease (AD) is a neurodegenerative disorder characterized by synaptic dysfunction, memory loss, neuroinflammation and neuronal cell death. Together with the genetic and neuropathological observations the experimental results have contributed to clarify the pathogenesis of AD. In this contest the development of transgenic mouse models engineered with over- expression of the amyloid precursor protein carrying familial AD mutations alone or in combination with PS1 or PS2 mutated transgene has been useful. Transgenic mice closely reproduce the typical human $\mathrm{AD}$ hallmarks, throughout aging they start to display impairment in synaptic activity associated to cognitive deficits. These phenomena precede plaque deposition. Once plaques start to be detectable in brain parenchyma, they appear surrounded by activated microglia and astrocytes as a signature of an active inflammatory response. At structural level, mice develop progressive brain atrophy in mostly involved areas such as the cortex, hippocampus and striatum reminiscent of the $\mathrm{AD}$ cerebral condition as documented by our lungitudinal MRI analysis. Although animal models are widely used to investigate mechanisms of pathology and efficacy of new therapeutic approaches they still miss some important aspects of the disease. For instance, AD therapeutic agents tested in transgenic mice provide most of the time encouraging results which, however, are very disappointing when transferred in clinical trials. Neuronal cell death are not easily detectable in mouse models, most likely for a matter of insufficient longevity. Also NFTs are much harder to replicate in these mice, unless in the presence of specific tau mutations which are, however, not observable in $\mathrm{AD}$ patients, rather in human taupathies. However, all together mouse models of AD certainly offer a fundamental tool for understanding and solving dementia pathology, but their improvement would undoubtedly represent an even greater achievement in the field. 


\section{The concept of MCI and its variants}

\author{
P. J. Nestor \\ German Center for Neurodegenerative Diseases \\ (DZNE) (Magdeburg-D)
}

The pivotal diagnostic criteria for Alzheimer's disease (AD), proposed over 30 years ago (McKhann et al, 1984), explicitly aimed at reducing falsepositive diagnoses. This was achieved, in part, by building a severity threshold into the criteria-patients had to perform below a cut-off on cognitive assessment, and, they had to have involvement of multiple cognitive domains. While this approach improved specificity for a diagnosis of $\mathrm{AD}$, as is often the case, the trade-off was sensitivity; it meant that a population of patients with Alzheimer pathology was excluded from the diagnosis in spite of being symptomatic. In particular, because people with symptomatic $\mathrm{AD}$, have relatively isolated memory impairment in the early stages, they fail to meet the multiple domain criterion. 'Mild cognitive impairment' (MCI) became the favoured term to capture this group using the operational criteria of informant-corroborated symptoms of memory dysfunction combined with objective neuropsychological evidence of memory impairment. The problem emerges, however, that as one moves towards milder degrees of impairment, the risk of false-positives increases such that not all who fulfill MCI criteria will have $\mathrm{AD}$ as the cause. This problem can now be largely circumvented by inclusion of amyloid biomarkers leading to the concept of MCI- stage AD. Aside from this 'amnestic MCI' category, the application of neuropsychological cut-offs also introduced the possibility of being impaired on a non-memory domain ('non-amnestic MCI') or being impaired in multiple cognitive domains ('multi-domain MCI'). Non-amnestic MCI tends to be a very unstable entity with follow-up - many having no definable degenerative pathology-while multi-domain MCI, unsurprisingly, tends to be highly associated with Alzheimer pathology.

\section{Clinical variants of Alzheimer dementia}

\section{S. F. Cappa \\ IUSS (Pavia)}

The concept of clinical variants (or "atypical") $\mathrm{AD}$ is certainly not new. The typical (i.e. episodic memory) presentation of $\mathrm{AD}$ is associated with the most common pattern of neuropathological progression, which, however, is not without exceptions (Gertz et al., 1998). "Focal" progressive neuropsychological syndromes characterized by prominent language or visuo-spatial impairment with preserved memory function have been increasingly recognized, and in a proportion of cases associated to Alzheimer pathology (Galton et al., 2000) . More recently, a set of diagnostic criteria have been defined for atypical AD, based on the definition of specific clinical phenotypes (posterior variant, logopenic variant, frontal variant, Down's syndrome variant) (Dubois et al., 2014). In line with the criteria for typical $\mathrm{AD}$, the diagnosis requires in-vivo evidence of $\mathrm{AD}$, as well as the exclusion of other conditions which may share clinical presentation with the variants. The diagnostic challenge is complex for all the variants, and requires the combination of in-depth cognitive and behavioural assessment with state of the art imaging techniques. The presentation phenotype faithfully reflects the neurodegeneration topography but bears only a probabilistic relationship with neuropathology. This may be relatively strong in the case of individual variants (posterior AD), but still largely undefined in the case of others (frontal variant). The role of pathophysiological biomarker is thus crucial for the diagnosis.

\section{References}

[1] Dubois, B. et al. Advancing research diagnostic criteria for Alzheimer's disease: the IWG-2 criteria. The Lancet Neurology (2014);13(6):614-629

[2] Galton, Clare J., et al. "Atypical and typical presentations of Alzheimer's disease: a clinical, neuropsychological, neuroimaging and pathological study of 13 cases." Brain 123.3 (2000): 484-498.

[3] Gertz HJ, Xuereb J, Huppert F, Brayne C, McGee MA, Paykel E, et al. Examination of the validity of the hierarchical model of neuropathological staging in normal aging and Alzheimer's disease. Acta Neuropathol (Berl) (1998); 95: 154

\section{Other dementias}

L. Bonanni, M. Onofrj

Department of Neuroscience Imaging and Clinical Sciences and CESI, University $G$ d'Annunzio of Chieti-Pescara (Chieti)

Dementia with Lewy bodies (DLB) is the second most common cause of degenerative dementia in elderly people after Alzheimer's disease (AD), with approximately $10-15 \%$ of dementia cases at autopsy demonstrating the presence of Lewy body pathology [1]. In Parkinson's disease (PD), dementia is a 
common outcome in up to $80 \%$ of patients with PD who will eventually develop dementia as their disease progresses $[2,3]$. DLB and PD can be grouped under the umbrella term of Lewy body disease (LBD) due to the overlap in symptom profile, similar response to treatment and the common underlying alpha-synuclein aggregation neuropathology [4]. Together DLB and Parkinson's disease with dementia (PDD) represent an important disease group in older age, with significant health and social impacts. LBD patients display marked deficits in executive and visuo-spatial/visuo-perceptual function, as well as marked variations in their level of arousal and attention which are known as cognitive fluctuations [5-8]. LBD clinical features also include spontaneous motor features of parkinsonism [6], and nonmotor manifestations including symptoms such as visual hallucinations, autonomic dysfunction, syncope, repeated falls, REM sleep behaviour disorder, delusions and depression [6]. However, there are a number of important diagnostic challenges. For example, the differentiation of DLB from Alzheimer's disease (AD) can be difficult, particularly in the early stages of disease when core DLB symptoms may be less evident [9]. Similarly, discrimination between PD, PD or DLB with mild cognitive impairment (PD-MCI, MCI-DLB) and PDD can be problematic as the development of cognitive impairment is often insidious and appears at a variable time after PD diagnosis [10]. There are also a number of treatment challenges. Profound cholinergic deficits occur in the LBDs, even more than in AD [11]. The use of cholinesterase inhibitors may have cognitive and neuropsychiatric benefits including improvements in global cognitive function, attentional function and activities of daily living [12]. However, there are frequently variations in treatment response [13]. The development of novel therapeutics is required, but this process has been hampered by the lack of LBD biomarkers which are sensitive to treatment response. In summary, therefore, there is a clear need to develop LBD biomarkers in order to facilitate early and differential diagnosis (during the prodromal phase of disease); improve our understanding of disease progression; provide a means to monitor therapeutic response; pave the way for early disease-modifying interventions. Existing biomarkers include a range of clinical, biochemical, genetic, proteomic, and, in particular, neuroimaging biomarkers $[17,18]$. Generally the utility of these biomarkers has only been supported in a research context aside from dopamine transporter imaging, which due to its high specificity in differentiating LBD from AD [19] is now considered the gold standard for in vivo differential diagnosis between LBD and AD. However FP-CIT remains expensive, has a radioactive exposure and provides little or no information on the disease progression, prognosis, or correlation with cognitive/behavioural symptom severity. Biomarkers should ideally be inexpensive, non-invasive, simple to use and technically validated [20]. In this regard, neurophysiological measures, and in particular electroencephalography (EEG), are currently undergoing a renaissance as potential biomarkers of neurodegenerative disease.

\section{References}

[1] McKeith IG: Consensus guidelines for the clinical and pathologic diagnosis of dementia with Lewy bodies (DLB): report of the Consortium on DLB International Workshop. J. Alzheimers Dis. (2006); 9:417-423

[2] Hely MA, Reid WGJ, Adena MA, et al: The Sydney multicenter study of Parkinson's disease: The inevitability of dementia at 20 years. Mov. Disord. (2008); 23:837-844

[3] Aarsland D, Andersen K, Larsen JP, et al: Prevalence and characteristics of dementia in parkinson disease: An 8-year prospective study. Arch. Neurol. (2003); 60:387-392

[4] Francis PT: Biochemical and pathological correlates of cognitive and behavioural change in DLB/PDD. J. Neurol. (2009);256 Supp1 3:280-285

[5] Mosimann UP, Mather G, Wesnes KA, et al: Visual perception in Parkinson disease dementia and dementia with Lewy bodies. Neurology (2004);63:2091-2096

[6] McKeith IG, Dickson DW, Lowe J, et al: Diagnosis and management of dementia with Lewy bodies: Third report of the DLB consortium. Neurology (2005);65:1863-1872

[7] Mollenhauer B, Forstl H, Deuschl G, et al: Lewy body and parkinsonian dementia: common, but often misdiagnosed conditions. Dtsch Arztebl Int (2010);107:684-691

[8] Lee DR, Taylor J-P,Thomas AJ: Assessment of cognitive fluctuation in dementia: a systematic review of the literature. Int. J. Geriatr. Psychiatry (2012);27:989-998

[9] Tiraboschi P, Salmon DP, Hansen LA, et al: What best differentiates Lewy body from Alzheimer's disease in earlystage dementia? Brain (2006);129:729-735

[10] Fernandez HH, Crucian GP, Okun MS, et al: Mild cognitive impairment in Parkinson's disease: the challenge and the promise. Neuropsychiatr Dis Treat (2005);1:37-50

[11] Samuel W, Caligiuri M, Galasko D, et al: Better cognitive and psychopathologic response to donepezil in patients prospectively diagnosed as dementia with Lewy bodies: a preliminary study. Int. J. Geriatr. Psychiatry (2000);15:794-802

\section{The brain safe mode: evidence from normal and pathological ageing}
G. Bottini ${ }^{1,2,3}$, G. Salvato ${ }^{1,2,3}$, M. Berlingeri ${ }^{2,3,4}$
${ }^{1}$ Department of Brain and Behavioral Sciences, University of Pavia (Pavia)
${ }^{2}$ Cognitive Neuropsychology Centre, ASST Grande
Ospedale Metropolitano Niguarda (Milano) 
${ }^{3}$ Neuromi, Milan Center for Neuroscience (Milano) ${ }^{4}$ DISTUM, Department of Humanistic Studies, University of Urbino Carlo Bo (Urbino)

It is well known that there is not a direct relationship between the degree of brain damage and its clinical manifestation across subjects. This empirical evidence is at the basis of the concept of cognitive reserve (CR). CR usually refers to individual differences either in cognition, or in neural networks that lead some people to cope better than others with neurocognitive damages, promoting a sort of brain safe mode. It arises from the interplay between a purely anatomical neural reserve and a more behavioral neural compensation process. In particular, recent empirical findings suggest that compensatory processes may manifest them-selves as additional recruitment of new neural networks in healthy aging. These scaffolding networks would be task-dependent and triggered by the specific level of task-demand. Interestingly, this form of age-related neural plasticity seems to get lost even in the prodromal phases of degenerative diseases such as in amnestic Mild Cognitive Impairment. Likewise, a number of studies have demonstrated that the brain may deal with the risk of developing neurodegenerative diseases, such as in the case of Apolipoprotein $\varepsilon 4$ (APOE $\varepsilon 4$ ) genotype, a condition typically associated with late- onset Alzheimer Disease (AD). The APOE $\varepsilon 4$ genotype and its influence on cognition may depend on age, gender, education, and lifestyle. Such variables would characterize the $\mathrm{CR}$, which can be addressed as a possible moderator between brain changes and the clinical manifestation of AD. These findings could be considered as potential tailored interventions in case of brain injuries or pathological ageing.

\section{Temporary binding: a promising cognitive marker for $\mathrm{AD}$}

\section{S. Della Sala}

Human Cognitive Neuroscience and Centre for Cognitive Ageing and Cognitive Epidemiology, Psychology Department, University of Edinburgh (Edinburgh-UK)

Accurate diagnosis of Alzheimer's Disease (AD) is essential for planning care, informing patients and carers, selecting patients for clinical trials, and assessing outcome of interventions. In clinical practice, diagnosis and monitoring of $\mathrm{AD}$ progression is aided by cognitive tests, which however are unreli- able for follow-up testing and only identify cognitive impairment, not the underlying disease causing the impairment. Memory impairment is the hallmark of $\mathrm{AD}$. However, memory impairment, as measured by current clinical assessment, is also affected by healthy ageing and chronic depression. There is an urgent unmet need for robust, reliable, cost-effective diagnostic tests to aid diagnosis and follow-up of patients with possible AD. We have developed a method that has been shown in multiple published studies to be specific to sporadic and familial AD relative to other dementias, to chronic depression and healthy ageing. The technique shows that individuals with $\mathrm{AD}$ have a specific problem in combining multiple sources of information, known as 'temporary binding' (TB). TB refers to the processes by which different aspects of stimuli such as colours and shapes are bound together on a temporary basis as an integrated object (i.e., a coloured shape). This particular binding task does not require learning, does not involve the hippocampus, is not affected by age, literacy or social background, does not show practice effects, it is inexpensive and it is easy and quick to administer. In sum, TB is a promising cognitive marker for $\mathrm{AD}$.

\section{Neuropsychological markers of progression in MCI}

\section{G. A. Carlesimo \\ S. Lucia Foundation and Systems Medicine \\ Department, Tor Vergata University (Roma)}

The early localization of neuropathological changes at level of mesio-temporal lobes (MTL) structures in typical Alzheimer's disease (AD) makes patients in the pre-clinical stages of the disease similar, with regard to the neuropsychological pattern of impairment, to patients with MTL amnesia. Indeed, the amnesic syndrome in these patients is characterized by a failure in consolidating new memory trace so that these patients are unable to recall or recognize recently experienced information, irrespective of the nature of memorandum or of the support to encoding and/or retrieval provided by the experimental paradigm. Conversely, other physiological or pathological conditions of the elderly (included other forms of dementia) do not present such precocious involvement of MTL structures so that their possible memory deficit is generally the consequence of a reduced functionality of frontal lobes (for direct involvement by pathological changes or for a deafferentation from other cortical or subcorti- 
cal projections). Similar to patients with focal frontal lobe damage, the memory impairment in these patients is not due to a failure in consolidating memory traces but, rather, to malfunctioning elaborative encoding of the incoming information and/or to failure in strategic retrieval of acquired data. Accordingly, these patients generally disclose very different memory performances depending on the nature of memorandum or the support to encoding and/or retrieval of the experimental task. Based on these premises, here we will review the studies which have been concerned with the distinction of MCI patients who convert or do not convert in $\mathrm{AD}$ based on their performance on episodic memory tests. Our aim is to provide experimental support to the above mentioned hypothesis that the qualitative profile of memory impairment in individuals with preclinical $\mathrm{AD}$ (deficit of memory trace formation and/or consolidation) differs from that of non-AD individuals (deficit mainly of elaborative encoding/strategic retrieval). For this purpose results of studies which have compared memory tests with short delay vs long delay intervals, memory tests for structured vs non structured material and, finally, memory tests with no help to encoding/retrieval vs memory tests which provided such help will be reviewed.

\section{Predictive tests for dementia}

\section{B. Dubois, S. Epelbaum}

Behavioral Neurology Unit (IM2A) and Dementia research center (ICM), Salpêtrière Hospital, University Pierre et Marie Curie (Paris-F)

Cognitive assessment is central to diagnosis and management of AD. Quantitative neuropsychological testing of the main cognitive domains (memory, executive functions and instrumental functions) ideally performed by someone trained in neuropsychology, should be considered in patients with questionable, prodromal, mild, or moderate dementia whereas it is less essential for severely demented patients. Episodic long-term memory impairment was recently proposed as central for the diagnosis of $\mathrm{AD}$ at least in the typical form of the disease (Dubois et al. Lancet Neurology, 2007 and 2014). To be recalled, the stimulus must go through three different and successive stages: 1) encoding, which is impaired in conditions that may interfere with attention processes: depression, anxiety, professional stress, sleep disorders, aging, treatment such as anticholinergic drugs or benzodiazepins... 2) storage, which relies on the hippocampus. In case of hippocampal lesions, such as in $\mathrm{AD}$, the perceived information cannot be stored as memory traces in long term memory; 3) and retrieval, which is impaired in frontal lesions (fronto-temporal dementias, subcorticofrontal dementias) and in functional states (depression or even normal aging where the activation of retrieval strategies is decreased). In summary, the best way to identify an amnestic syndrome of the hippocampal type, as in $\mathrm{AD}$, is to assess memory by tests that control for attention and that can facilitate retrieval. Using memory tests with cueing, either at bedside (5-Word Test) (Dubois et al. 2002) or by neuropsychologist (Free and Cued Selective Reminding Test) (Grober et al. 1988) is recommended because of their good specificity AD (Mormont et al. 2012, Wagner et al. 2012, Dierckx et al. 2009). Other memory tests, particularly those based on list learning and delayed recall, can also be effective in identification of the amnestic syndrome of AD. These tests include the paired-associate learning, the Rey auditory verbal learning tasks and the shortterm memory binding test (Estevez-Gonzalez et al. 2003, Fowler et al. 2002, Lowndes et al. 2008, Della Sala et al. 2012).

\section{The meeting of informatics with brain medicine: a new concept for biomarkers - disease signatures}

\section{R. Frackowiak \\ HBP \& EPFL (Lausanne-CH)}

Single human gene mutations may present with any of multiple phenotypes, and vice versa, a range of genetic abnormalities may cause a single disease phenotype. A major initial research aim of the Medical Informatics Platform of the Human Brain Project is to use big data techniques and algorithms to fill the multi-dimensional brain disease space, which ranges from psychiatry through behavioural disorders to neurological diseases, with groups of similar patients characterised by combinations of homogeneous features. We call such a group of specific features a "disease signature". A disease signature brings together, in a systematic way, clinical features (phenomenology) and the results of genetic, biological, physiological and anatomical test results (biology). This reclassification should in the longterm supplant the symptom and syndrome based DSM and ICD disease catalogues by ones based on the same clinical features supplemented by patterns 
of abnormal investigations. This strategy has as its primary aim the definition of more precise diagnoses that reduce error variance in diagnostic categories for construction of clinical trial cohorts. A biological underpinning of phenomenological features may in combination with improved understanding of the functional and structural organisation of the human brain emanating from other parts of the Human Brain Project facilitate aspects of brain medicine such as identification of biological treatment targets, planning of potential benefits, risks and side effects of proposed treatments, as well as considerable reduction of clinical cohort sizes associated with more precise disease signature based definitions of diagnosis and prognosis. These ideas will be illustrated with reference to the human dementias.

\section{Pre-clinical biomarkers - Imaging Biomarkers}

\section{Structural MRI}

Federica Agosta

Neuroimaging Research Unit, Institute of

Experimental Neurology, Division of Neuroscience, San Raffaele Scientific Institute (Milano)

Thanks to evolving biomarker research and substantial discoveries, it is now possible to identify Alzheimer's disease (AD) (and other dementia) even at the preclinical stage before the occurrence of the first clinical symptoms. Abnormalities in structural MRI become clearly detectable before the first clinical signs of AD. Rate of longitudinal changes assessed by structural MRI increases with progression to clinical disease in cognitive intact $A \beta$ positive elderly individuals and can be used for staging of high risk of progression. An increase in brain atrophy were detected 15 years before expected symptom onset in subjects with autosomal dominant AD. A recent, large, international cohort study of families with autosomal dominant frontotemporal dementia showed that atrophy of insula and temporal lobe can be identified 10 years before expected onset of symptoms in asymptomatic adults at risk of genetic FTD. Emerging evidence from networkbased neuroimaging studies, such as diffusion tensor MRI studies, have implicated specific large-scale brain networks in the pathogenesis of neurodegenerative dementia, suggesting a new paradigm for explaining the distributed and heterogeneous spreading patterns of pathological proteins in these conditions. Preliminary studies suggest that characterizing brain network breakdown in these presymptomatic or asymptomatic "at-risk" individuals using MRI may help anticipate the diagnosis and perhaps prevent the devastating impact of neurodegenerative diseases.

\section{A clinical role for fMRI in dementia: is the long wait over?}

\section{E. Paulesu \\ Psychology Department \&NeuroMI, University of Milano-Bicocca, IRCCS Galeazzi (Milano)}

Since their inception, the development of every imaging technique has been motivated also by the urgency of finding new answers to neurological disorders, dementias being one of the major challenges in modern times. fMRI is no exception. In spite of the tremendous technological progress in fMRI research, a practical role in dementia clinics is still missing for several reasons: on the one hand any advance in the identification of the disorders of their precursor conditions has been frustrated by the lack of any game changer in the therapy of dementia; on the other hand it remains unclear on whether taskbased or resting state connectivity [1] fMRI studies are better suited to capture the transition from normal aging into conditions prodromal to dementia. Another unanswered question is how these techniques may fare in comparison with 18F-FDG PET or PET scanning of amyloid distribution. In addition, it is clear that a single index, as much as technological as it might be, it is most likely insufficient to represent a reliable biomarker of the disease and of the response to any treatment $[2,3]$. In my communication I will briefly compare the achievements of task-based fMRI studies and resting state connectivity studies in dementia and in ageing. I will argue that, for the time being, none of the findings of these technologies on their own can represent the ideal biomarker that clinicians may seek to make clinical decisions when it comes to individuals. However, if incorporated into a sophisticated labelling procedures of the clinical and neurological status of a given patient [4], fMRI data may give a stronger contribution. For example, much as for other complex disorders (e.g. Gilles de la Tourette syndrome [5]), I envisage that pre-surgical fMRI profiling of patients candidate to deep brain stimulation (may this prove to be of some help in dementia) may help in deciding at the individual level who are the best 
candidates to the therapy. This may also apply to pharmacological treatments. This, however, has a long road ahead of clinical experimentation, for each and every candidate therapy, before turning into practice.

\section{References}

[1] Sheline Y. and Raichle ME. Resting state functional connectivity in preclinical Alzheimer's disease. Biol Psychiatry (2013);74:340-347

[2] Bottini G., et al. GOOD or BAD responder? Behavioural and neuroanatomical markers of clinical response to donepezil in dementia. Behavioural Neurology (2012); 25(2):61-72

[3] Katsnelson A. Life sciences: Biomarkers on the brain. Nature (2011);479(7371): 139-40

[4] Kloppel S. et al. Automatic classification of MR scans in Alzheimer's disease. Brain (2008);131:681-689

[5] Zapparoli L. et al. The anarchic brain in action: the contribution of task-based fMRI studies to the understanding of Gilles de la Tourette syndrome. Curr Opin Neurol. 2015 Dec;28(6):604-11

\section{Cross validation of imaging biomarkers in clinical settings. The crucial role of FDG-PET imaging}

\author{
D. Perani \\ Vita-Salute San Raffaele University, Nuclear \\ Medicine Unit San Raffaele Hospital, Division of \\ Neuroscience San Raffaele Scientific Institute \\ (Milano)
}

Background: Although the use of research diagnostic criteria can improve dementia diagnosis at the individual level, it may be still challenging for clinicians. Effective strategies supporting for more certain early and differential diagnosis are needed for a better management of dementia, fitting in clinical trials and treatment options.

Aims: Evaluating in clinical-settings the supportive role of PET biomarkers for the early and differential diagnosis of dementia.

Methods: Large series of patients with neurodegenerative dementia, atypical parkinsonisms, and mild cognitive impaired (MCI) subjects with a long clinical follow-up assessed with conventional brain MRI, optimized voxel-based FDG-PET, and PET measures of amyloid load.

Results: SPM FDG-PET showed the highest diagnostic accuracy (AUC $\approx 90 \%$ ), providing both high sensitivity and specificity in the early and differential diagnosis of dementia and parkinsonisms, and it was the best predictor of conversion from MCI to $\mathrm{AD}$ or other dementias in MCI. PET Amyloid mea- sures allowed to exclude AD pathology and showed some added value in MCI subgroups.

Conclusion: The SPM FDG-PET analysis at the individual level allows accurate early and differential diagnosis in neurodegenerative conditions, also in the prodromal disease phase, with a high disease exclusionary role.

\section{Cerebrospinal fluid $A \beta$ and tau}

\section{Parnetti \\ Section of Neurology, Centre for Memory \\ Disturbances, University of Perugia, Perugia}

Amyloid $\beta 1-42$, total tau and phosphorylated tau represent the three core CSF biomarkers for Alzheimer's disease. Each of them correlates to one of the key characteristics of Alzheimer's disease pathology: low levels of CSF amyloid- $\beta 1-42$ correlate with greater plaque load, high levels of total tau correlate with greater intensity of neuronal degeneration, and high levels of phosphorylated tau correlate with neurofibrillary tangle pathology. The presence of low levels of amyloid- $\beta 1-42$ in combination with high levels of total tau and phosphorylated tau provide support for $\mathrm{AD}$ diagnosis with a sensitivity of 80-93\% and specificity of $82-90 \%$ against cognitively normal controls, and high total tau and low amyloid $\beta 1-42$ are most pronounced at more advanced stages. With respect to other dementia disorders, Alzheimer's patients display the highest levels of total tau and phosphorylated tau, combined with low levels of amyloid- $\beta 1-42$. Recent studies carried out in large independent cohorts of patients have shown that the use of the simple ratio of tau to Ab42 for differentiating $\mathrm{AD}$ patients from other patients and for predicting dementia due to AD in MCI patients represents a robust and generalizable parameter. Basically, in routine clinical context, the optimal CSF Alzheimer profile is whenever tau is more than half the value of Ab42. The use of the ratio is advantageous since its diagnostic performance is superior to what observed in single biomarkers, and also may overcome the differences observed among laboratories in terms of cut-off and absolute values.

\section{References}

[1] Duits FH et al. The cerebrospinal fluid "Alzheimer profile": Easily said, but what does it mean? Alzheimer's \& Dementia (2014);10:713-723.

[2] Skillbäck T et al. Cerebrospinal fluid tau and amyloid-b1-42 in patients with dementia. Brain (2015);138:2716-2731. 


\section{Anti-abeta antibodies in CSF: markers for therapies?}

\author{
F. Piazza \\ Coordinator of The inflammatory Cerebral Amyloid \\ Angiopathy and Alzheimer's disease Biomarkers \\ (iCA $\beta$ ) International Network \& The iCA $\beta$-ITALY \\ Study Group of the Italian Society for the study of \\ Dementia (SINdem) \\ School of Medicine and Surgery, Milan Center for \\ Neuroscience (NeuroMi), University of Milano- \\ Bicocca (Monza)
}

Advances in the biomarkers discovery for Alzheimer's disease (AD) and Cerebral Amyloid Angiopathy (CAA) has sensibly accelerated the design of novel disease-modifying therapies (DMT), with different promising anti amyloid-beta $(A \beta)$ therapeutic antibodies already in Phase II and III. Active and passive immunotherapies, however, have been both characterized by the occurrence of Amyloid-Related Imaging Abnormalities (ARIA), probably related to the drug and APOE 44 allele dose. In the last decade, ARIA have severely limited the development of immunotherapy, leading to the exclusion of several patients from the opportunity to be treated. The discovery of safety biomarkers to avoid, or at least enable, the early detection of ARIA will represent an important challenge to help stratify and personalize treatments, increasing the chances for developing more effective DMT. Biomarkers will have critical implications to predict individuals in a particular disease stage chosen as the therapeutic window for a specific treatment, especially as we move to more large and long duration prevention trials based on the selective enrolment of positive Amyloid-PET and/or CSF cases, potentially increasing the risk to incur in ARIA side effects of treatment. It is quite clear that without effective biomarkers we will have the consequence of further unacceptable delays in finding a cure for this devastating disease. Through the iCA $\beta$ International Network, we pioneered in showing that elevated CSF anti-A $\beta$ autoantibodies are linked to a transient vascular leakage at the sites of major $A \beta$ removal, causing a shift in CAA accumulation and increased vascular permeability, eventually leading to ARIA.

\section{Blood biomarkers}

E. Scarpini

Neurology Unit, Department of Pathophysiology

and Transplantation, University of Milan,

Fondazione Ca' Granda, IRCCS Ospedale

Policlinico (Milano)

So far, no blood biomarkers for Alzheimer's disease (AD) validated against pathology exist. Among candidates there are circulating proteins and a novel class of molecules, namely non coding RNA, which includes micro(mi)RNA, which are 18-22nt long small regulatory RNA. They are able to bind to the target messenger RNA and inhibit its translation. They play their regulatory role in cells, but can be found freely circulating in body fluids, including plasma and cerebrospinal fluid or in exosomes, particularly those of neural derivation. In this presentation, the current knowledge on the role of miRNA in $\mathrm{AD}$ and related dementias will be discussed, together with their potential future usefulness as peripheral biomarkers.

\section{Longitudinal imaging and biochemical assessments}

M. Marizzoni ${ }^{1}$, S. Galluzzi ${ }^{1}$, C. Ferrari ${ }^{1}$, J. Jovicich ${ }^{2}$, F. Nobili ${ }^{3}$ J.-P. Ranjeva ${ }^{4}$, D. BartrésFaz $^{5}$, U. Fiedler ${ }^{6}$, P. Schönknech ${ }^{7}$, P. Payoux ${ }^{8,9}$, A. Beltramello ${ }^{10}$, M. Caulo ${ }^{11}$, A. Soricelli ${ }^{12,13}$, L. Parnetti ${ }^{14}$, M. Tsolaki ${ }^{15}$, P. M. Rossini ${ }^{16,17}$, P. Jelle Visser $^{18}$, D. Albani ${ }^{19}$, G. Forloni ${ }^{19}$, R. Bordet ${ }^{20}$, J. Richardson ${ }^{21,22}$, O. Blin ${ }^{23}$, G. B. Frisoni ${ }^{1,24}$; on behalf of the PharmaCog Consortium ${ }^{1}$ Laboratory of Neuroimaging and Alzheimer's Epidemiology, IRCCS Istituto Centro San Giovanni di Dio Fatebenefratelli (Brescia)

${ }^{2}$ Center for Mind/Brain Sciences, University of Trento (Trento)

${ }^{3}$ Department of Neuroscience, Ophthalmology, Genetics and Mother-Child Health (DINOGMI), University of Genoa (Genova)

${ }^{4}$ CIC-UPCET, CHU La Timone, AP-HM, UMR CNRS-Universite de la Mediterranee (Marseille-F)

${ }^{5}$ Department of Psychiatry and Clinical

Psychobiology, Universitat de Barcelona and IDIBAPS (Barcelona-E)

${ }^{6}$ LVR-Clinic for Psychiatry and Psychotherapy, Institutes and Clinics of the University DuisburgEssen (Essen-D) 
${ }^{7}$ University Hospital Leipzig (Leipzig-D)

${ }^{8}$ INSERM, Imagerie cérébrale et handicaps neurologiques, UMR 825 (Toulouse-F)

${ }^{9}$ Université de Toulouse, UPS, Imagerie cérébrale et handicaps neurologiques, (Toulouse-F)

${ }^{10}$ Department of Neuroradiology, General Hospital (Verona)

${ }^{11}$ University "G. d'Annunzio" of Chieti (Chieti)

${ }^{12}$ IRCCS SDN (Napoli)

${ }^{13}$ University of Naples Parthenope (Napoli)

${ }^{14}$ Section of Neurology, Centre for Memory

Disturbances, University of Perugia (Perugia)

${ }^{15} 3 \mathrm{rd}$ Department of Neurology, Aristotle University of Thessaloniki (Thessaloniki-GR)

${ }^{16}$ Dept. Geriatrics, Neuroscience \& Orthopaedics, Catholic University, Policlinic Gemelli (Roma)

${ }^{17}$ IRCSS S.Raffaele Pisana (Roma)

${ }^{18}$ Alzheimer Center and Department of Neurology,

VU University Medical Center (Amsterdam-NL)

${ }^{19}$ Neuroscience Department, IRCCS Istituto di

Ricerche Farmacologiche "Mario Negri"

(Milano)

${ }^{20}$ Department of Pharmacology, EA1046,

University of Lille Nord de France, 59045 Lille

Cedex (Lille-F)

${ }^{21}$ Neurosciences Therapeutic Area U.K. (United

Kingdom)

${ }^{22}$ GSK R\&D, China-UK, U.K. (United Kingdom)

${ }^{23}$ Pharmacology, Assistance Publique-Hôpitaux de

Marseille, Aix-Marseille University-CNRS UMR

7289 (Marseille- F)

${ }^{24}$ Memory Clinic and LANVIE - Laboratory of

Neuroimaging of Aging, University Hospitals and

University of Geneva (Geneva-CH)

Background: Markers sensitive to the progression of Alzheimer's disease (AD) in its pre-dementia stages are important to develop disease modifying drugs.

Aim: To identifying biomarkers sensitive to disease progression in amnestic mild cognitive impairment (aMCI) patients classified into positives or negatives based on their baseline CSF A $\beta 42 /$ ptau level.

Methods: 147 aMCI patients were enrolled in WP5 of PharmaCog (E-ADNI). ADAS-cog, structural, diffusion and functional MRI were performed each 6 months for at least 2 years. Patients converted to $\mathrm{AD}$ or other type of dementia were excluded from follow-up visits.

Statistical analysis: Linear Mixed Model with repeated ADAS-cog and MRI measures as dependent variables; time and CSF A $\beta 42 / p$-tau status as independent variable; age, gender, total intracranial volume (volume models) as covariates.

Results: The ADAS-cog score showed effects for CSF status and time-CSF status interaction $(p<0.001)$ but not for time. Effect for CSF status, time and their interaction was reported for volumes (hippocampus including several subfields, lateral ventricles, thalamus), entorhinal cortex, and for diffusivity in the fornix (all $p<0.025$ ). The explained variability of ADAS-cog, volumes and fornix diffusivity was 0.54 , higher than 0.82 and 0.35 , respectively. Finally, rs-fMRI measures (e.g. default mode network connectivity) reported effect only for CSF status $(\mathrm{p}<0.045)$.

Conclusions: Structural MRI measures are the most sensitive to change over time even in a mildly progressing aMCI population. Volume measures were better than ADAS-cog in separating A $\beta 42 / \mathrm{p}$ tau positive relative to negative aMCI patients in the 2-year period.

Pharmacog is funded by the EU-FP7 for the Innovative Medicine Initiative (grant $n^{\circ} 115009$ ).

\section{Randomized, Placebo-Controlled, Phase 1b study of the anti-Beta-amyloid antibody Aducanumab (BIIB037) in patients with prodromal or Mild Alzheimer's Disease: interim results}

V. Viglietta ${ }^{1}$, L. Williams ${ }^{1}$, T. Chen ${ }^{1}$, J. O'Gorman ${ }^{1}$, P. Chiao' ${ }^{1}$, C. Hock ${ }^{2}$, R. M. Nitsch², A. Sandrock ${ }^{1}$

${ }^{1}$ Biogen (Cambridge-USA)

${ }^{2}$ Neurimmune Holding $A G$ and University of Zurich (Zurich-CH)

Aducanumab is a human monoclonal antibody against aggregated forms of beta-amyloid $(\mathrm{A} \beta)$ being investigated as a disease-modifying treatment for patients with Alzheimer's disease (AD). The ongoing Phase $1 \mathrm{~b}$ PRIME study is evaluating the safety, tolerability, pharmacokinetics, and pharmacodynamics of multiple doses of aducanumab in patients with prodromal or mild AD. In this multicenter, randomized, double-blind, placebo-controlled, multipledose study, patients (age 50-90 years) had positive florbetapir (Amyvid) positron emission tomography (PET) scans and met clinical criteria for prodromal or mild AD. In a parallel staggered cohort design, patients were randomized to receive aducanumab $(1,3$, 6 , or $10 \mathrm{mg} / \mathrm{kg}$ or dose titration) or placebo (ratio of 
3:1 active versus placebo) once every 4 weeks for 52 weeks within 9 treatment arms stratified by ApoE4 status. The primary endpoint assessed safety and tolerability; secondary endpoints included pharmacokinetic parameters, presence of anti-aducanumab antibodies, and change from baseline to Week 26 in amyloid plaque burden, as measured by PET imaging. Changes in clinical measures of cognitive decline (including change from baseline in Clinical Dementia Rating, sum of boxes [CDR-sb] and MiniMental State Examination [MMSE] scores) were assessed in exploratory endpoints. We report interim safety, $A \beta$ reduction by amyloid PET, and exploratory clinical endpoints (changes from baseline in CDR-sb and MMSE). Patients $(\mathrm{N}=166)$ were randomized to placebo $(\mathrm{n}=40$, pooled), $1(\mathrm{n}=31), 3$ $(n=33), 6(n=30)$, or $10(n=32) \mathrm{mg} / \mathrm{kg}$ aducanumab. The most common adverse events (AE) were amyloid-related imaging abnormalities (incidence as detected by MRI: $10 \%, 16 \%, 33 \%$, and $47 \%$ for $1,3,6$, and $10 \mathrm{mg} / \mathrm{kg}$ aducanumab, respectively, versus $5 \%$ for placebo) and headache $(16 \%, 16 \%, 27 \%$, and $28 \%$ versus $5 \%$ ). Treatment- related dose- and timedependent reductions in brain amyloid plaque burden (as shown by SUVR ratio reduction at Week 26 and further at Week 54) were observed within the doses tested. Dose-dependent attenuation of cognitive decline was observed with aducanumab in exploratory clinical endpoints. Dose-dependent ARIA was the main safety and tolerability finding. Aducanumab reduced amyloid plaque burden as measured by PET imaging in patients with prodromal or mild AD. A clinical signal was observed in exploratory analysis.

This study is funded by Biogen.

\section{Amyposomes: a nanomedicine designed for treatment of Alzheimer disease}

M. Masserini ${ }^{1}$, S. Mancini ${ }^{1}$, G. Sancini ${ }^{1}$, C. Balducci ${ }^{2}$, G. Forloni' ${ }^{2}$, F. Re ${ }^{1}$

${ }^{1}$ Nanomedicine Center and School of Medicine, University Milano Bicocca (Milano)

${ }^{2}$ Department of Neuroscience, IRCCS, Istituto di Ricerche Farmacologiche "Mario Negri," (Milano)

A consistent body of evidence suggests that a pivotal role in the pathogenesis of Alzheimer disease $(\mathrm{AD})$ is played by $\beta$-amyloid (A $\beta)$. Currently, the therapy of $\mathrm{AD}$ is an unmet medical need. A possible contribution to solve this problem is offered by nanotechnology, with the possibility to design all-in-one nanodevices carrying different molecular components, whose cooperativity allows the accomplishment of a multitask action. Within this frame we previously designed, and patented, a nanotechnology taylored to dismantle $A \beta$ aggregates and remove brain Abeta burden. The technology is based on Amyposomes, multi- functionalized nanoliposomes that proved in vitro the ability to bind Abeta with high affinity, to inhibit its aggregation, to destroy Abeta assemblies and to cross blood-brain barrier models without showing neuron toxicity. We evaluated the efficacy of Amyposomes on different $\mathrm{Tg}$ rodent models of AD, namely APP/PS1 or APP23 mice. The results suggest that Amyposomes, systematically administered, are able to enter the mice brain with effects either on brain $A \beta$ burden or on memory impairment. The treatment is effective on "old" Tg mice upon acute treatment, promoting the decrease of brain $A \beta$ and the amelioration of memory impairment, or on "young" mice upon long-term treatment, slowing down brain $\mathrm{A} \beta$ accumulation and memory impairment. Together, these data indicate Amyposomes as a new nanothecnological device potentially suitable for AD treatment. UNIMIB has taken the decision to spin-off the project and develop the technology. The Spin-off company Amypopharma is seeking for investors to carry Amyposomes to clinical phase, going through completion of the preclinical development and IMPD filing.

\section{Mediterranean Diet}

\author{
N. Scarmeas \\ National and Kapodistrian University of Athens, \\ Columbia University (Atene - GR, New York- \\ USA)
}

Background: There has been considerable research on the relation between nutrition and risk for Alzheimer's disease but the results have been conflicting. Among the various methodological reasons for non-consistent results, the examination of individual foods or nutrients (vs. Dietary patterns) stands as an important one because we do not consume foods in isolation but as part of an overall diet.

Aims: To review the relevant literature and describe future perspectives

Methods: We briefly outline previous attempts of looking into dietary patterns in relation to cognition. We present some of our data on the association between a Mediterranean-type diet (MEDI) and Mild 
Cognitive Impairment (MCI), Alzheimer's disease (AD). We also summarize some of our recent studies investigating potential biological mechanisms mediating the association between MEDI and cognitive performance.

Results: The majority of the literature so far suggests that higher adherence to this diet is associated with lower risk for developing AD and MCI. Biological mechanisms of possible associations between MEDI and cognition may include vascular, anti-infammatory, antioxidative and metabolic pathways while associations with more direct AD related pathology such as amyloid and neurodegeneration is also conceivable.

Conclusions: Exploring diet via holistic approaches may provide additional useful insights into potential relations with cognitive outcomes and may result in information important from a public health perspective. Much work in relation to biological mechanisms needs to be performed. Most of the so far evidence derives from observational epidemiological studies and clinical trials are a necessary next step.

\section{Cognitive Stimulation Therapy (CST) for dementia}

\section{A. Spector \\ Research Department of Clinical, Educational and Health Psychology, University College (London- $U K)$}

CST is a brief, evidence-based intervention for people with mild to moderate dementia. Complex psychological techniques are embedded within simple, manualised group sessions which aim to stimulate and maximise cognitive skills. These include categorisation, word association and discussion of current affairs, all following guiding principles which encourage the making of new semantic connections to aid learning. More recently, an individualised, carer-led CST programme has been developed and evaluated. This talk will provide an overview of the CST research programme and key findings from three clinical trials. This includes standard CST, longer-term CST and individualised CST (iCST). In summary, changes following group CST may be comparable to those associated with anti- dementia drugs and it is cost-effective. Memory, executive functioning and language were significantly improved, and significant benefits in quality of life were maintained for six months. Individualised CST led to significant improvements in one's relationship with their caregivers, although cognition did not improve - suggesting the importance of delivery within a group format. The CST research programme has led to changes in service provision in the UK. Government guidelines now recommend routine provision of Cognitive Stimulation, regardless of medication use and as the only non-pharmacological intervention for the cognitive symptoms of dementia. CST provision is now a key standard for memory service accreditation and used in over $66 \%$ of UK memory clinics. The World Alzheimer's Report recommends routine provision of CST in early stage dementia, resulting in its use globally. The manual has been translated into numerous languages, including Italian.

\section{Magnetic stimulation}

\section{Leocani, R. Chieffo \\ Dept. of Neurology and INSPE-Institute of Experimental Neurology, Hospital San Raffaele (Milano)}

Alzheimer's disease (AD), leading to progressive loss of memory, language and several other cognitive functions, representing the leading cause of dementia with has a strong socio-economic impact consequences. Considering that there are few available pharmacological therapies, other interventions, such as cognitive/behavioural therapy and repetitive transcranial magnetic stimulation (rTMS) are currently explored. In particular, rTMS is recognized as a promising intervention for treating motor and cognitive deficits in several neurological disorders, owing to its ability to modulate cortical activity beyond the stimulation period through mechanisms of brain plasticity. Although the available evidence on $\mathrm{AD}$ is overall conflicting, recent reports point to the advantage of combining rTMS with cognitive training, possibly owing to converging mechanisms of the two interventions on the same cognitive networks. Another potential improvement in this approach is the consideration that several and widespread brain networks are affected by the disease, potentially requiring the application of stimulation of several targets, with sequential focal stimulation or, as allowed by recent technological advances, with newly designed $\mathrm{H}$-coils allowing a wider area of stimulation compared with traditional focal coils. Further stud- 
ies are needed specifically addressing the possible advantage of other combination approaches, such as dietary or exercise. Another unmet need in the use of rTMS is the identification of factors predictive of a positive response, with particular focusing on cognitive and brain reserve, towards the identification of the best candidates with the goal of precision medicine and tailored interventions in AD.

\section{Modulating the human hypothalamus with Deep Brain Stimulation}

\author{
A. M. Lozano \\ University Professor and Canada Research Chair, \\ University of Toronto (Toronto-CND)
}

Deep Brain Stimulation (DBS) is being used to modulate the activity of motor and limbic circuits in the treatment of movement disorders and psychiatric illness. More recently, we have applied DBS in the fornix to study its effects in the context of a clinical trial or Alzheimer's disease. We have noted that the delivery of electrical stimulation here can produce striking clinical effects including in some cases, memory-related phenomenon. The rationale and preliminary observations seen with hypothalamic/ fornix stimulation in our clinical trial for Alzheimer's disease and in animal models will be reviewed. 


\section{Extracellular Vesicles Isolated From Bone Marrow Mesenchymal Stem Cells Induce An Anti-Inflammatory Phenotype In Microglia Exposed To Human Abeta 1-42}

C. A. Elia' ${ }^{1}$ S. Marchetti ${ }^{2}$, M. Losurdo ${ }^{2}$,

A. Saccomano ${ }^{2}$, F. Filipello', M. Rasile ${ }^{1}$,

M. Tamborini ${ }^{1}$, M. Matteoli ${ }^{3}$, S. Coco ${ }^{2}$

${ }^{1}$ Laboratory of Pharmacology and Brain

Pathology, Humanitas Clinical and Research

Center, IRCCS (Rozzano-MI)

${ }^{2}$ Dept. Medicine and Surgery, University of Milano

Bicocca (Monza)

${ }^{3}$ Institute of Neuroscience, CNR, National Research

Council (Milano)

In Alzheimer's disease (AD), the presence of brain senile plaques, neurofibrillary tangles and activated microglial cells strongly contribute to generate an inflammatory environment. The aim of the project is to investigate a possible anti-inflammatory effect of bone marrow derived mesenchymal stem cell microvesicles (BM-MSC MVs) in microglia cultures exposed to human beta Amyloid peptide 1-42 (h-Abeta 1-42). BM-MSCs isolated from 4-12 week old C57BL/6 mice were expanded in vitro and tested for their ability to differentiate into specific mesenchymal lineages. BM-MSC MVs were isolated from the culture medium, by performing a series of centrifugations and then characterized by using FACS and Western Blot analysis for specific surface markers. Primary cultures of murine microglial cells, derived from post-natal day 2 mice, were treated with h-Abeta1-42 peptide in the presence of BM-MSC MVs or MV- deprived culture supernatant, to evaluate the expression of different inflammatory or antiinflammatory markers by immunocytochemistry and cytokine production by ELISA. BM-MSC MVs down-regulate the inflammatory processes induced by exposure of microglia to $\mathrm{h}$ Abeta 1-42 peptide in vitro. In fact, microglial cells, following MV treatment, assume an amoeboid phenotype becoming less ramified and releasing anti-inflammatory cytokines without significantly affecting the release of the pro-inflammatory ones. Moreover, MVs lead to a decreased expression of MHC II, associated with a pro-inflammatory phenotype. As in AD the chronic pro-inflammatory environment is postulated to contribute to the cognitive deficits and neuronal loss that characterize the disease, the immunomodulatory properties of BM-MSC MVs may represent a powerful tool to modulate this aberrant process.

\section{Neuroinflammation correlates with Hippocampus Atrophy in Alzheimer's Disease}

M. Saresella ${ }^{1}$, F. Baglio 2 , M. Cabinioº ${ }^{2}$ F. Piancone ${ }^{1}$, F. Larosa ${ }^{1}$, I. Marventano ${ }^{1}$, E. Calbrese ${ }^{3}$, R. Nemni ${ }^{3,4}$, M. Clerici ${ }^{1,4}$

${ }^{1}$ Laboratory of Molecular Medicine and Biotechnology, Don C. Gnocchi Foundation IRCCS (Milano)

${ }^{2}$ Laboratory of Imaging Department of Neurology, Don C. Gnocchi Foundation IRCCS (Milano) ${ }^{3}$ Department of Neurology, Don C. Gnocchi Foundation IRCCS (Milano)

${ }^{4}$ Department of Physiopathology and Transplants, University of Milano (Milano)

The pathogenesis of Alzheimer's disease (AD) is characterized by two alterations that are the consequence of b-amyloid (Abeta) accumulation: neuroinflammation and hippocampus atrophy. We investigated possible correlations between these two AD-associated phenomena using structural Magnetic Resonance Imaging (MRI) to evaluate hippocampal volume (Hv) and immunologic analyses to analyze neuroinflammation. Results obtained in 30 $\mathrm{AD}$ individuals showed the presence of statistically significant: 1) positive correlations between either Left or Right Hv and circulating anti-inflammatory monocyte; 2) positive correlations between Left Hv and Abeta-stimulated CD14+ IL-10+ cells or TGFbeta+ or CD200R+; 3) negative correlation between Left $\mathrm{Hv}$ and circulating inflammatory monocyte or Abeta-specific-CD14+IL-6+ or IL-23+ cells; 4) negative correlations between either Left or Right $\mathrm{Hv}$ and Abeta-RORgamma+ CD4+T cells; 5) negative correlations between Left Hv and Abeta-IFNgamma CD4+ T cells. These correlations are immunologically sound as: 1) circulating anti-inflammatory monocyte, interleukin -10 and TGFbeta slow-down 
immune response and inflammation; 2) the interaction of CD200 protein with CD200R, inhibits immune stimulatory responses 3) circulating inflammatory monocyte, IL-6 and IL-23 elicit immune response; 4) CD4+ RORgamma $+\mathrm{T}$ and CD4+IFNgamma cells identify the pro-inflammatory Th17 and Th1 subsets respectively of circulating lymphocytes. The observation that reduced Hv correlates with an impairment of anti-inflammatory Abeta-specific peripheral monocytes and, conversely, with a higher percentage of circulating proinflammatory monocyte and of Th1, Th17 Abeta-specific lymphocytes strongly suggest that neuroinflammation and hippocampus atrophy are indeed correlated in $\mathrm{AD}$ and indicate that monitoring of immune cells in peripheral blood could have a prognostic value in this disease.

\section{Sirtuins modulation as a novel therapeutic strategy in in vitro and in vivo models of Alzheimer's Disease}

\author{
F. Fusco, G. Biella, A. Agrillo, D. Albani, \\ G. Forloni \\ Department of Neuroscience, IRCCS Mario Negri \\ Institute for Pharmacological Research (Milano)
}

Aims: Alzheimer's disease (AD) is the most prevalent neurodegenerative disorder with cognitive decline. Several data support the involvement of the deacetylase sirtuins (SIRTs) in neurodegeneration. Particularly, SIRT2 was reported to promote neurodegeneration, while SIRT1 seemed to be neuroprotective. Our aim is to evaluate SIRT2 inhibition as novel therapeutic approach in $\mathrm{AD}$, also in synergy with SIRT1 activation.

Methods: In vitro treatments (SIRT2 inhibitors AGK2/AK7; SIRT1 activators resveratrol (RSV)/ SRT1720) were performed on H4-SW/H4-native human neuroglioma cells. For in vivo tests we selected APP23 and 3xTg-AD mouse models, treated with the brain-permeable SIRT2 inhibitor AK7. Cognitive performance was assessed by novel object recognition test (NORT). For AD-relevant biochemical parameters, we performed Western Blotting (WB) or Immunohistochemistry.

Results: SIRT2 inhibition affected in vitro APP metabolism, reducing soluble APP (sAPP) fragments and $A \beta-40 / A \beta-42$ peptides. We performed a cellular treatment with AK7 in combination with RSV to verify the potential toxicity, and cellular vi- ability was unaffected by the treatment. In vivo, AK7 therapy counteracted the cognitive deficit revealed in Tg-vehicle mice by NORT. WB demonstrated an increased level of sAPP $\alpha$, reduced sAPP $\beta$, unchanged flAPP production and increased total tau expression, but no effect on neuroinflammatory markers (GFAP/IBA1).

Discussion: Our data suggest a positive role of SIRT2 inhibition in AD and support the possibility of a combined therapy on SIRT1/SIRT2 to look for a synergistic effect.

Conclusions: In conclusion, SIRTs modulation seems to be a useful tool, and finding an optimal brain-delivery protocol could be a step forward in $\mathrm{AD}$ research.

\section{References}

[1] Donmez G, Outeiro TF. SIRT1 and SIRT2: emerging targets in neurodegeneration. EMBO Mol Med. (2013) Mar;5(3):344-52

[2] Donmez G. The Effects of SIRT1 on Alzheimer's Disease Models. Int J Alzheimers Dis. (2012); 2012:509529

[3] Green KN, Steffan JS, Martinez-Coria H, Sun X, Schreiber SS, Thompson LM, LaFerla FM. Nicotinamide restores cognition in Alzheimer's disease transgenic mice via a mechanism involving sirtuin inhibition and selective reduction of Thr231-phosphotau. J Neurosci. (2008) Nov 5;28(45):1150010

\section{Multiplex ligation-dependent probe amplification (MLPA) assay in PSEN1 and APP genes in early-onset Alzheimer disease patients}

I. Ricca ${ }^{1}$, G.S. Grieco ${ }^{1}$, M. Valente ${ }^{1}$, P. Bini ${ }^{2}$, P. Vitali ${ }^{3}$, C. Cereda ${ }^{1}$

${ }^{1}$ Center of Genomics and post-Genomics, Mondino National Institute of Neurology Foundation (Pavia)

${ }^{2}$ Neurological Department, Mondino National Institute of Neurology Foundation (Pavia)

${ }^{3}$ Neuroradiology and Brain MRI Mondino Research Center, Mondino National Institute of Neurology

Foundation (Pavia)

Mutations in PSEN1 gene account for $30-70 \%$ of cases of early-onset familial Alzheimer disease (EOFAD). More than 200 mutations are reported in PSEN1, spanning throughout the coding sequence. Exonic rearrangements are thought to be very rare with the exception of the deletion involving exon 9 in the Finnish population. These rearrangements can be missed using conventional sequencing techniques. To date, there are few reports about the use 
of quantitative analysis, such as MLPA or qPCR, in the screening of EOFAD. We performed MLPA analysis of PSEN1 and APP genes on a group of 7 EOAD cases and we found one deletion of exon 7. The deleted patient presented global cognitive decline, disorientation and depression starting from the age of 60. Family history was unremarkable. 3T MRI showed mild ventricular enlargement, bilateral moderate mesial temporal atrophy and diffuse cortical atrophy, more prominent in the left parietal lobe. Cerebral blood flow map by Arterial Spin Labeling showed predominant hypoperfusion within the parietal lobe. This is the first report of exon 7 deletion in a patient affected by EOAD. Exon 7 contains a catalytic residue implicated in the active site of gammasecretase, so deletions of this part of the PSEN1 gene could affect the protein function [1]. The neuroradiological pattern is typical for early onset $\mathrm{AD}$, with predominant parietal over temporomesial atrophy [2]. Our work highlights the importance of studying exonic rearrangements in $\mathrm{AD}$ genes and suggests that these mutations could be more frequent than expected (1/7 in our sample).

\section{References}

[1] Shen J, Kelleher RJ III. The presenilin hypothesis of Alzheimer's disease: evidence for a loss-of-function pathogenic mechanism. PNAS (2007) Jan 9;104(2):403-9

[2] Frisoni G. Patterns of cortical and subcortical atrophy in early-onset and late-onset Alzheimer's disease. Alzheimer's \& Dementia (2011) July; 7(4):S89-S90

\section{Comparison of molecular biomarkers for the prediction of conversion to dementia in a large multicentric MCI cohort}

\author{
S. Caminiti ${ }^{1}$, T. Ballarini ${ }^{1}$, L. Presotto ${ }^{1}$, A. Sala ${ }^{1}$, \\ C. Cerami ${ }^{2}$, F. Fallanca ${ }^{3}$, EG. Vanoli ${ }^{3}$, D. Perani ${ }^{3}$, \\ F. Nobili ${ }^{4}$, L. Parnetti ${ }^{5}$, G. Frisoni ${ }^{6}$, E. Scarpini ${ }^{7}$ \\ ${ }^{1}$ Division of Neuroscience, San Raffaele Scientific \\ Institute (Milano) \\ ${ }^{2}$ Clinical Neuroscience Department, San Raffaele \\ Scientific Institute (Milano) \\ ${ }^{3}$ Nuclear Medicine Unit, San Raffaele Scientific \\ Institute (Milano) \\ ${ }^{4}$ Department of Neuroscience, Ophthalmology, \\ Genetics and Mother-Child Health (DINOGMI), \\ University of Genoa (Genova) \\ ${ }^{5}$ Section of Neurology, Centre for Memory \\ Disturbances, University of Perugia (Perugia)
}

\author{
${ }^{6}$ IRCCS Foundation San Giovanni Di Dio - \\ Fatebenefratelli (Brescia) \\ ${ }^{7}$ Department of Neurology, University of Milan \\ (Milano)
}

Aim: This multicentre study aimed at comparing the accuracy of FDG-PET and CSF biomarkers in predicting conversion to dementia.

Materials: N=99 MCI-subjects (mean-age $70.42 \pm 7.3$ ) were evaluated.

Methods: We applied an optimized SPM method [1-2], obtaining single-subjects FDG-PET SPM-t maps. Each patient SPM-t map was classified by imaging experts, blind to clinical information, as negative, AD-like, DLB-like or FTD-like patterns. Pathological CSF-values were defined on the basis of the standard cut-off [3]. Multivariate Cox regression analysis was performed in order to assess biomarkers prediction accuracy.

Results: The $84 \%$ SPM-t maps were classified as pathological (33\% AD-like, 33\% FTD-like, 17\% DLB-like), and, at follow-up (20.2 \pm 10 months), the $55 \%$ MCI among those converted to dementia of different types. $16 \%$ MCI showed normal metabolism and none of them converted to dementia after follow-up (15\% were MCI-stable and 1\% MCI-reverter). As for CSF, 77\% MCI showed alterations in at least two CSF-biomarkers, and among those the $53 \%$ converted to AD. Of note, $15 \%$ MCI with normal CSF-values converted to $\mathrm{AD}$ at follow-up. Cox regression analysis indicated as most accurate predictor for dementia conversion FDG-PET SPM t-maps $(\exp \beta=28.7, \quad \mathrm{CI}=1.14$ 722.74, $\mathrm{p}<0.05 ;$ Sens $=100 \%$ and $\mathrm{Spec}=65 \%$ ), whereas the CSF biomarkers did not survive the statistical threshold.

Discussion: FDG-PET SPM t-maps classification had the highest predictive value in identifying patterns of brain hypometabolism characteristic of different neurodegenerative diseases and normal metabolism in MCI-stable or reverter.

Conclusion: The high predictive power of FDGPET SPM method supports its role in clinical settings for risk evaluation of dementia progression in the early prodromal phase.

\section{References}

[1] Perani D., Della Rosa P.A., Cerami C., Gallivanone F., Fallanca F., Vanoli E.G., Panzacchi A., Nobili F., Pappatà S., Marcone A., Garibotto, V. Validation of an optimized SPM procedure for FDG-PET in dementia diagnosis in a clinical setting. NeuroImage: Clinical (2014);6:445-454 
[2] Perani D., Cerami C., Caminiti S.P., Santangelo R., Coppi E., Ferrari L., Pinto P., Passerini G., Falini A., Iannaccone S., Cappa S.F. Cross-validation of biomarkers for the early differential diagnosis and prognosis of dementia in a clinical setting. European journal of nuclear medicine and molecular imaging, (2015):1-10.

[3] Tapiola, T., Alafuzoff, I., Herukka, S.K., Parkkinen, L., Hartikainen, P., Soininen, H. and Pirttilä, T., 2009. Cerebrospinal $\beta$-amyloid 42 and tau proteins as biomarkers of Alzheimertype pathologic changes in the brain. Archives of Neurology (2009);66(3):382-389

\section{Memory impairment in AD and PDD: hippocampal subfields involvement and category cued recall}

F. Novellino ${ }^{1}$, R. Vasta ${ }^{1}$, G. Nicoletti ${ }^{1}$, C. Chiriaco ${ }^{1}$, M. Vaccaro ${ }^{2}$, M. Morelli², M. Salsone' ${ }^{1}$, G. Arabia ${ }^{2}$,

A. Sarica ${ }^{2}$, A. Quattrone 2

${ }^{1}$ Institute of Bioimaging and Molecular Physiology,

National Research Council (Catanzaro)

${ }^{2}$ Institute of Neurology, University "Magna

Graecia" (Catanzaro)

Objective: We investigated how changes in whole hippocampus and hippocampal subfields relate to memory recall in different forms of dementia, as Alzheimer's Disease(AD) and Parkinson's Disease with Dementia(PDD).

Methods: We compared 22 AD, 18 PDD, 21 PD, and 17 healthy subjects through a combination of a multimodal MRI hippocampal subfields evaluation and a memory assessment with the Free and Cued Selective Reminding Test (FCSRT), a hippocampaltargeted neuropsychological paradigm. Volume, mean diffusivity (MD) and fractional anisotropy (FA) of automatically segmented hippocampus (whole and subfields) were obtained. Correlations between MRI-derived metrics and clinical and neuropsychological evaluations were performed.

Results: ANOVA yielded a strong overall difference among the groups in the entire hippocampus in volume and $\mathrm{MD}$, showing a grading in the increase of both hippocampal atrophy (left: $\mathrm{F}=8.237 /$ $\mathrm{p}<0.0001$; right: $\mathrm{F}=9.650 / \mathrm{p}<0.0001)$ and $\mathrm{MD}$ values (left: $F=17.713 / p<0.0001 ;$ right: $F=15.696 / p<0.0001$ ) according to the order $\mathrm{AD}>\mathrm{PDD}>\mathrm{PD}>$ controls. In the subfields analyses, the comparison between $\mathrm{AD}$ and PDD groups revealed a significant volume reduction in the right subiculum ( $\mathrm{p}=0.02)$, an increased $\mathrm{MD}$ in the bilateral CA1 (right $\mathrm{p}=0.004$; left $\mathrm{p}=0.016)$, left presubiculum $(\mathrm{p}=0.015)$, right CA4$\mathrm{DG}(\mathrm{p}=0.018)$ and subiculum $(\mathrm{p}=0.013)$. Correlation analyses showed significant correlation between
FCRST scores and hippocampal subfields measurement in AD group.

Discussion and conclusion: Our results show that hippocampal subregions have different vulnerability to AD-linked damage. Subiculum, CA4DG and presubiculum were the regions more strictly involved in $\mathrm{AD}$ and related to cued recall. These results may help explain how memory processing is associated with the function of specific hippocampal subfields and improve the knowledge of specific AD-related mnesic profile.

\section{References}

[1] Dubois, B., Feldman, H.H., Jacova, C., et al. Research criteria for the diagnosis of Alzheimer's disease: revising the NINCDS ADRDA criteria. Lancet Neurol. (2007):6;734-746

[2] Sarazin M, Chauviré V, Gerardin E, et al. The amnestic syndrome of hippocampal type in Alzheimer's disease: an MRI study. J Alzheimers Dis. (2010);22:285-94

\section{"Mitochondrial Alterations in}

\section{Peripheral Blood Mononuclear Cells} (PBMCs) from Alzheimer's Disease and Mild Cognitive Impairment Patients: new potential blood biomarkers for $A D$ "

\author{
M. Marziano, G. Abate, D. Uberti \\ DMMT, University of Brescia (Brescia)
}

Objective: Evaluation of different mitochondrial markers in Peripheral Blood Mononuclear Cells (PBMCs) of Alzheimer's Disease (AD) and Mild Cognitive Impairment (MCI) subjects as possible $\mathrm{AD}$ biomarkers. Materials: AD, MCI and healthy subjects were enrolled and their PBMCs were isolated.

Methods: The expression of mitochondrial markers were evaluated with quantitative rtPCR and immunoblotting. Mitochondrial DNA was measured by qPCR. Cytochrome $\mathrm{C}$ oxidase and citrate synthase activity was also measured by enzymatic assays.

Results: Cytochrome $\mathrm{C}$ and cytochrome $\mathrm{B}$ were decreased in $\mathrm{AD}$, while $\mathrm{MCI}$ showed only a reduction of cytochrome C. AD and MCI exhibited highly nitrated MnSOD. TFAM, a regulator of mitochondrial genome replication and transcription, was decreased in both patient groups while PGC- $1 \alpha$, that regulates the expression of nuclear encoded mitochondrial factors, was reduced only in $\mathrm{AD}$. Also the mtDNA amount was reduced in both patient groups. 
Discussion: We found some differences and some similarities between $\mathrm{AD}$ and MCI when compared with healthy subjects. For example, cytochrome $\mathrm{C}$ and cytochrome $\mathrm{B}$ were decreased in $\mathrm{AD}$, while MCI showed only a statistical reduction of cytochrome C. On the other hand, both $\mathrm{AD}$ and $\mathrm{MCI}$ blood cells exhibited highly nitrated MnSOD, index of a prooxidant environment inside the mitochondria. TFAM, was decreased in both AD and MCI. Moreover also the mitochondrial DNA amount was reduced in PBMCs from both patient groups.

Conclusion: These data confirmed peculiar peripheral mitochondrial impairment in $\mathrm{AD}$ and $\mathrm{AD}$ progression.

\section{References}

[1] Delbarba A, Abate G, Prandelli C, Marziano M, Buizza L, Arce Varas N, Novelli A, Cuetos F, Martinez C, Lanni C, Memo M, Uberti D. Mitochondrial Alterations in Peripheral Mononuclear Blood Cells from Alzheimer's Disease and Mild Cognitive Impairment Patients. Oxid Med Cell Longev. (2016);2016:5923938 doi:10.1155/2016/5923938. Epub 2016 Jan 6. PubMed PMID: 26881032; PubMed Central PMCID: PMC4736772.

[2] P. H. Reddy and M. F. Beal Amyloid beta, mitochondrial dysfunction and synaptic damage: implications for cognitive decline in aging and Alzheimer's disease. Trends in Molecular Medicine (2008);14(2):45-53

[3] S. Hauptmann, I. Scherping, S. Dröse et al. Mitochondrial dysfunction: an early event in Alzheimer pathology accumulates with age in $\mathrm{AD}$ transgenic mice. Neurobiology of Aging (2009);30(10):1574-1586

\section{A cognitive preventive intervention by means of Memory Training (MT) in a sample of older adults aged $65+$}

\author{
M. Veneziano ${ }^{1}$, C. Prete ${ }^{1}$, B. Senesi ${ }^{1}$, M. Puntoni ${ }^{2}$, \\ M. Gianelli ${ }^{3}$, A. Pilotto ${ }^{1}$ \\ ${ }^{1}$ Centre for Cognitive Disorders and the Dementias \\ (CCDD), Geriatric Unit, Geriatric Care \\ Department, Orthogeriatrics and Rehabilitation, \\ Galliera Hospitals (Genova) \\ ${ }^{2}$ Clinical Experimentation Planning and \\ Management, Biostatistics. Scientific Coordinator's \\ Office, Galliera Hospitals (Genova) \\ ${ }^{3}$ University Academic Researcher, INTERDEM \\ (Genova)
}

Aims: Primary goal of the study is to prove the efficacy of MT in a 930 subject, cognitively intact, aged 65-80. Secondary goals: how participants evaluate themselves and their cognitive performances and depressive symptoms.

Materials and Methods: The project is in collaboration with Regione Liguria. All participants in the study were evaluated via a clinical conversation and administration of neuropsychological screening tests. Inclusion criteria: age between 65 and 80 years; absence of psychiatric pathologies; absence of sensorial deficits; absence of dementia. Evaluation tests for cognitive status include: MMSE1 and CDT2. The cognitive stimulation programme includes: 1,30 minutes meetings, once a week, over three months. Each meeting include a maximum of 20 participants. At baseline, at conclusion of intervention is measured: cognitive status, by learning test of three word lists 3 and verbal fluency by letter (Tognoni,1986); self-perception of cognitive functionality by MACQ (Crook,1992); depression by GDSsv (Almeida,1999).

Results: The score of the outcomes at conclusion of intervention presented a statistically significant improvement with respect to base values.

Conclusions: The study proves that the MT produce significant improvement of cognitive status, self-perception of cognitive wellness by participants and reduction the depressive symptoms. The results to suggest that the use of MT is feasible even into an advanced age, and that the improvement of cognitive activity, mood status and social well-being are protective factors with respect to cognitive deterioration. MT is to be considered primary social health preventive interventions, in their capacity of slowing down the negative effects of cerebral aging.

\section{References}

[1] Magni E, Binetti G, Bianchetti A, Rozzini R, Trabucchi M. Mini-Mental State Examination: a normative study in Italian elderly population. Eur J Neurol. (1996) May;3(3):198-202

[2] Sunderiand T, Hill JL, Mellow AM et al. Clock drawing in Alzheimer's disease. A novel measure of dementia severity. J Am Geriatr Soc (1989);37:725-9

[3] Novelli G., Papagno, C., Capitani E., Laiacona M. Three clinical tests for the assessment of verbal long-term memory function: Norms from 320 normal subjects. Archivio di Psicologia, Neurologia e Psichiatria (1986); 47(/2): 278-296 


\section{Evaluation of the acceptability of a caring service robot (MARIO)}

G. D’Onofrio'1, O. James², D. Sancarlo', F. Ricciardi ${ }^{3}$, K. Murphy ${ }^{2}$, F. Giuliani ${ }^{3}$, D. Casey², A. Greco ${ }^{1}$

${ }^{1}$ Geriatric Unit \& Laboratory of Gerontology and Geriatrics, Department of Medical Sciences,

Fondazione "Casa Sollievo della Sofferenza" (San Giovanni Rotondo-FG)

${ }^{2}$ National University of Ireland (Galway-IRL)

${ }^{3} I C T$, Innovation and Research Unit, Fondazione

"Casa Sollievo della Sofferenza" (San Giovanni

Rotondo-FG)

Objectives: In the frame of the European Community funded MARIO project, the caregivers of 139 dementia patients were recruited in National University of Ireland (NUIG), in Geriatrics Unit of IRCCS "Casa Sollievo della Sofferenza"-Italy (IRCCS) and in Alzheimer Association Bari-Italy (AAB) for a multicenter survey to determine the needs and preferences of caregivers to improve the assistance of patients with dementia and to guide the technological development of the MARIO companion robot.

Materials and Method: A six minute video on technological devices and functions of MARIO was showed, and all caregivers fulfilled a 43-item questionnaire that explored four areas: A) Acceptability, B) Functionality, C) Support devices, and D) Impact.

Results: Caregivers declared that facilitating acceptance (over 17.5\%) and improving functionality of MARIO (over 29\%) should be important/likely/ useful. Over $20.3 \%$ of caregivers reported that following support devices in MARIO could be useful for their patients: 1) to monitor bed-rest and movements, 2) to monitor medication use, 3) to monitor the ambient environmental conditions, 4) to regulate heating, humidity, lighting and TV 5) to undertake comprehensive geriatric assessment, 6) to link to care planning, 7) to monitor physiological deterioration, and 8) to monitor cognitive deterioration. Over $21.8 \%$ of caregivers declared that MARIO should be useful to improve quality of life, quality of care, safety, emergency communications, home-based physical and/or cognitive rehabilitation programs, and to detect isolation and health status changes of their patients.

Conclusions: MARIO is a novel approach employing companions robot and its effect will be: 1) to facilitate and support persons with dementia and their caregivers, and 2) to reduce social exclusion and isolation. 


\section{CLINICAL MANAGEMENT}

\section{Cognitive stimulation therapy in Italian patients: a pilot study}

\author{
M. Balestrino, S. Terzuoli, L. Bonaretti, D. Sassos, \\ F. Donato \\ Dept. of Neuroscience, University of Genoa \\ (Genova)
}

Goal: We carried out a pilot study to investigate the feasibility and possible efficacy of Cognitive Stimulation Therapy (CST) for Alzheimer's type dementia in Italian patients.

Patients: Seven patients aged (mean \pm SD) $80 \pm 4$ years, affected by Alzheimer's type dementia.

Methods: We patterned our intervention after published protocols of CST (Spector et al., 2006). To this aim we created suitable materials in Italian. We delivered 15 group sessions twice a week. To each patient we administered the following tests before and after CST: Mini Mental State Examination (MMSE), Geriatric Depression Scale (GST), Alzheimer's Disease Assessment Scale-cognitive subscale (ADAS-Cog), Neuropsychiatric Inventory (NPI).

Results: We found no change after CST in MMSE. Similarly, we found no change in GDS, however 4 out of 5 patients that had a GDS $>5$ at baseline (suggestive of clinically relevant depression) showed improvement after CST. Both ADAS-Cog and NPI improved after CST.

Discussion and Conclusions: The results are in agreement with clinical trials (e.g., Niu et al., 2010) and with one Cochrane meta-analysis of CST (Woods et al., 2012). They showed significant improvement in non-cognitive symptoms of dementia (BPSD cluster) and in caregiver burden of care, while some improvement was shown in cognitive symptoms as well. They support the need for a fullypowered, controlled study on the efficacy of CST in Italian patients.

\section{References}

[1] Spector A, Thorgrimsen L, Woods B, et al. Making a difference: An evidence-based group programme to offer Cognitive
Stimulation therapy (CST) to people with dementia. Hawker Publications: London, UK (2006)

[2] Niu YX, Tan JP, Guan JQ, et al. Cognitive stimulation therapy in the treatment of neuropsychiatric symptoms in Alzheimer's disease: a randomized controlled trial. Clin Rehabil. (2010); 24(12):1102-11

[3] Woods B, Aguirre E, Spector AE, et al. Cognitive stimulation to improve cognitive functioning in people with dementia. Cochrane Database Syst Rev. (2012) 15;2:CD005562

\section{All for one and one for all: a novel approach to single case studies with VBM}

C. De Santis ${ }^{1}$, M. Berlingeri², M. Tettamanti ${ }^{3}$, G. Bottini ${ }^{4}$, M. Sberna ${ }^{5}$, E. Paulesu ${ }^{1}$

${ }^{1}$ Department of Psychology, University of Milano-

Bicocca (Milano)

${ }^{2}$ Department of Humanistic Studies (DISTUM),

University of Urbino Carlo Bo (Urbino)

${ }^{3}$ Division of Neuroscience and Department of

Nuclear Medicine, IRCCS San Raffaele Scientific

Institute (Milano)

${ }^{4}$ Department of Brain and Behavioral Sciences, University of Pavia (Pavia)

${ }^{5}$ Neuroradiology Department, Niguarda Ca'

Granda Hospital (Milano)

Objectives: We report a new method to assess whether Voxel Based Morphometry (VBM) is reliable for comparisons between individual patients and control groups.

Materials: We included 121 healthy subjects and 3 patients with probable diagnosis of, respectively, $\mathrm{AD}, \mathrm{FTD}$, and PPA.

Methods: For each healthy subject, we generated 4 sets (defined by 4 sample-sizes: 8, 16, 24, and 32) of 10 control groups using a bootstrapping without replacement method. For each subject and sample size, we performed a two-sample t-test (1st level). We applied a one-sample t-test on the 10 first-level contrast images of each subject by sample size (2nd level). Finally, we applied a one-sample t-test on the 2nd-level contrast images of all subjects by sample size ( $3 \mathrm{rd}$ level). We quantified the false positive rate, ranging from uncorrected .05 to uncorrected .000001 , with decile steps, plus .05 FWE corrected. For the 3 patients, we used the same 1 st and 2 nd 
level analyses, and used both an uncorrected .001 and a FWE corrected .05 thresholds.

Results: For healthy subjects, no significant voxels survived the 3 rd level one sample t-tests. For the 3 patients, consistent overlapping effects (at least $6 / 10$ ) were only observed at .001 uncorrected with control group sizes of 16 or larger.

Discussion: Our VBM bootstrap sampling approach resolves previously reported problems of false positives and unreliable results [1].

Conclusion: The empirical validation with three patients showed that our method seems to be sensible enough to detect regions of consistent brain atrophy, even with relatively small control samples of sizes.

\section{Reference}

[1] Scarpazza, C. et al., 2013, When the single matters more than the group: Very high false positive rates in single case Voxel Based Morphometry. Neuroimage (2013);70:175-188

\section{Adult-onset idiopathic epilepsy in presymptomatic Alzheimer's disease}

J. C. Di Francesco ${ }^{1}$, V. Polonia ${ }^{1}$, L. Tremolizzo ${ }^{1}$, E. Beghi², G. Giussani², E. Bianchi² ${ }^{2}$ I. Appollonio ${ }^{1}$, C. Ferrarese ${ }^{1}$

${ }^{1}$ Dept. of Neurology, San Gerardo Hospital, Milan Center for Neuroscience, School of Medicine and

Surgery, University of Milano-Bicocca (Milano, Monza)

${ }^{2}$ Laboratory of Neurological Disorders, IRCCS Istituto di Ricerche Farmacologiche "Mario Negri" (Milano)

Alzheimer's disease (AD) is associated with an increased risk of epilepsy. Seizure activity has been interpreted as a secondary process of neurodegeneration. The prevalence of epilepsy beginning in the adulthood increases with age, mainly due to the accumulation of brain damage (vascular, tumor, traumatic). However, a significant proportion of patient experience "idiopathic" epilepsy, thus without a recognizable cause. Since the neurodegenerative processes leading to dementia begin several years before clinical symptoms, seizure could be more frequent in the presymptomatic stages of the disease. We retrospectively reviewed the prevalence of epilepsy/seizures BEFORE the onset of cognitive symptoms in a large cohort of neurodegenerative dementias (EPS-DEM) recruited at the UVA Center, San Gerardo Hospital, Monza (globally 2300 subject, mainly AD). Clinical, EEG, NRx and NPS data were collected and compared with dementias NOT associated with epilepsy (noEPS-DEM) and with a large reference population (CT), matched for age and gender (Giussani, Epilepsia 2014). The prevalence of idiopathic, adult-onset epilepsy before cognitive decline is 2-3 times more frequent in the group of EPS-DEM compared with CT. Moreover, cognitive symptoms begins approximately 4 years earlier in EPS-DEM, compared with noEPSDEM. Neurodegenerative processes of dementia could play a key role in the pathogenesis of epilepsy in individuals intended in the future to develop cognitive decline. Epilepsy of undefined cause with onset in the adulthood could thus represent an early biomarker of the neurodegenerative damage, even preceding by several years the onset of cognitive symptoms.

\section{Reference}

[1] Giussani G, Franchi C, Messina P, Nobili A, Beghi E; EPIRES Group.Prevalence and incidence of epilepsy in a well-defined population of Northern Italy. Epilepsia. (2014) Oct;55(10):1526-33. doi:10.1111/epi.12748

\section{Adaptation of Cognitive Stimulation Therapy in the Italian context}

S. Gardini ${ }^{1}$, S. Faggian ${ }^{2}$, S. Pradelli ${ }^{3}$, E. Salvalaio ${ }^{2}$, E. Morciano ${ }^{1}$, G. Michelini ${ }^{1}$, P. Caffarra ${ }^{1}$, E. Borella ${ }^{4}$

${ }^{1}$ Department of Neurosciences, University of Parma (Parma)

${ }^{2}$ Centro Servizi Anni Sereni (Scorze, VE)

${ }^{3}$ inVentiv Health Clinical (Princeton - USA)

${ }^{4}$ Department of General Psychology, University of

Padua (Padova)

Objectives: Cognitive Stimulation Therapy (CST) is an effective and person-centred intervention for People with Dementia (PWD) in the mild to moderate phase. This study aimed to adapt CST in the Italian context and to observe the effects of this intervention on cognitive domains, social cognition, narrative language and mood.

Materials: PWD were recruited in Residential Care Homes, had mild to moderate dementia (Inclusion criteria Spector et al., 2003). Participants were randomly assigned to the experimental (CST) and control group (Treatment As Usual).

Methods: Pre- and post-test assessments included tools evaluating general cognitive abilities, social 
cognition (theory of mind), language, working memory, functional abilities, behavioural and psychological symptoms (NPI, social and emotional loneliness), and quality of life. The CST group took part to fourteen group sessions, twice a week with two facilitators, whereas the control group continued the usual activities of the centre, such as reading newspapers, cards games.

Results: After treatment, the CST group, compared with the control one, showed a significant improvement in ADAS-COG subscales scoring the linguistic domains, social cognition and depression symptoms. Participants and facilitators also reported good qualitative ratings about CST.

Conclusions: CST benefits were confirmed. They were found, for the first time in specific domain such as in the theory of mind and narrative language. The application of CST in the Italian context also showed a good response in PWD and operators.

Discussion: The present findings sustain the applicability of CST in the Italian context. Multi-center randomized controlled study is in course.

\section{References}

[1] Spector A, Thorgrimsen L, Woods B, Royan L, Davies S, Butterworth $\mathrm{M}$ and Orrell M. Efficacy of an evidence-based cognitive stimulation therapy programme for people with dementia: Randomised Controlled Trial. British Journal of Psychiatry (2003); 183: 248-254

[2] Spector A, Thorgrimsen L, Woods B and Orrell M. Making a difference: An evidence-based group programme to offer Cognitive Stimulation therapy (CST) to people with dementia. Hawker Publications: UK. (2006).

[3] Aguirre E, Spector A, Orrell M. Guidelines for adapting cognitive stimulation therapy to other cultures. Clin Interv Aging (2014);9:1003-7

\section{The development of a Home-based and Computerized Cognitive Stimulation Therapy for people living with Dementia: preliminary results}

\author{
S. Gardini, G. Michelini, P. Tirelli, E. Morciano, \\ P. Caffarra \\ Department of Neurosciences, University of Parma \\ (Parma)
}

Objectives: Cognitive Stimulation Therapy is an evidence-based and effective intervention in mild to moderate dementia. This study aimed to develop a Home-based and Computerized version of CST to be performed by the Person With Dementia (PWD) with the support of the caregiver.
Materials: The group CST protocol (14 structured sessions) was adapted for the computerized administration, respecting the CST guiding principles, and then implemented on a Tablet. A manual of instructions and toolkit (e.g. lyrics book, map of Italy) were provided with the Tablet. A group of Person with Dementia and caregivers took part to this pilot study.

Methods: All the fourteen sessions are usable through a Tablet, with Android v. 4.4 as operating system, and define an Android application (shortly APP), developed in JAVA. Each session is introduced by a video where an operator speaks about the daily activities, followed by Welcome, Karaoke, Reality Orientation and News, the main activity of the day and the concluding part. Neuropsychological assessment was carried out before and after treatment.

Results: The platform is user-friendly and satisfactory for the PWD and caregiver. After treatment, PWD had significant increase of performance in some ADAS-COGS subscales (word recall, commands, orientation), functional abilities (DAD total and execution), and caregiver reported a higher sense of competence.

Conclusions: The home-based and computerized CST application is a complete and suitable platform for PWD.

Discussion: The developed computerized version of CST seems to be a promising tool in offering CST at home to PWD including the caregiver.

\section{References}

[1] Spector A, Thorgrimsen L, Woods B, Royan L, Davies S, Butterworth M and Orrell M. Efficacy of an evidence-based cognitive stimulation therapy programme for people with dementia: Randomised Controlled Trial. British Journal of Psychiatry (2003);183: 248-254

[2] Spector A, Thorgrimsen L, Woods B and Orrell M. Making a difference: An evidence-based group programme to offer Cognitive Stimulation therapy (CST) to people with dementia. Hawker Publications: UK (2006)

[3] García-Casal JA, Loizeau A, Csipke E, Franco-Martín M, Perea-Bartolomé MV, Orrell M. Computer-based cognitive interventions for people living with dementia: a systematic literature review and meta-analysis. Aging Ment Health (2016);25:1-14 
Preliminary results of a randomized controlled trial about the effectiveness of eye movement desensitization and reprocessing integrative group treatment protocol (EMDR-IGTP) for the reduction of psychological distress in caregiver of patients with dementia

\author{
S. Passoni ${ }^{1}$, T. Curinga ${ }^{1}$, A. Toraldo ${ }^{2}$, \\ M. Berlingeri ${ }^{3}$, I. Fernandez ${ }^{4}$, G. Bottini ${ }^{1}$ \\ ${ }^{1}$ Cognitive Neuropsychology Center, A.S.S.T \\ Grande Ospedale Metropolitano Niguarda \\ (Milano) \\ ${ }^{2}$ Department of Brain and Behavioural Sciences, \\ University of Pavia (Pavia) \\ ${ }^{3}$ Psychology Department, University of Milano- \\ Bicocca (Milano) \\ ${ }^{4}$ E.M.D.R. Italy Association, C.R.S.P. sas (Bovisio \\ Masciago-MB)
}

Background: The assistance to a person with dementia can be considered as a traumatic event, resulting in rise in the level of psycho-physical stress. The World Health Organization recommend Eye Movement Desensitization and Reprocessing therapy (EMDR) for treatment of trauma. It focuses on trauma elaboration, with the use of alternating bilateral stimulation. Recently, some researchers have tried to apply EMDR even to a group context, developing the EMDR Integrative Group Treatment Protocol (EMDR-IGTP) (Jarero e Artigas, 2009).

Aims: To evaluate the effectiveness of the EMDRIGTP in reducing caregivers' distress and caregivers' emotional symptoms compared to a control group.

Methods: The protocol combines the eight phases of the EMDR Individual Therapy Treatment in a group therapy model, that uses the Butterfly Hug (Artigas \& Jarero, 2014), as a self-administered bilateral stimulation. The EMDR-IGTP intervention consisting of eight group sessions of 120 minutes each, once a week. Measures about caregiver distress symptoms were collected before and after the group treatment.

Results: A significant differential improvement in the EMDR-IGTP with respect to the control group in the expected direction was found in three out of five of the tested measures: Anxiety $(\mathrm{F}=18.928$, onetailed $\mathrm{p}<.001)$, Depression $(\mathrm{F}=4.091$, one-tailed $\mathrm{p}=.0355)$, and Burden $(\mathrm{F}=4.111$, on-tailed $\mathrm{p}=.035)$. The other two scales, Impact of Event Scale-Revised and Caregiver Needs Assessment led to one-tailed p-values of .053 and .077 respectively.

Conclusions: In spite of a very small sample size ( $\mathrm{N}=6$ per group), our preliminary results witness a significant contribution of EMDR-IGTP to a reduction of anxiety, depression and burden.

\section{References}

[1] Jarero I., Artigas L. EMDR integrative group treatment protocol. Journal of EMDR Practice \& Research (2009);3(4):287288

[2] Artigas L., Jarero I., Alcalá N., López Cano T. The EMDR Integrative Group Treatment Protocol (IGTP) for Children. In M. Luber (Ed.). Implementing EMDR Early Mental Health Interventions for Man-Made and Natural Disasters (pp. 237251). New York, NY: Springer. (2014)

\section{A diagnostic algorithm based on cerebrospinal fluid biomarkers and neuropsychological tests can easily and reliably predict progression from mild cognitive impairment to Alzheimer's dementia}

R. Santangelo ${ }^{1}$, S. Mazzeo ${ }^{1}$, G. Cecchetti ${ }^{2}$, P. Pinto ${ }^{3}$, M. Bernasconi ${ }^{1}$, M. Falautano ${ }^{1}$, G. Passerini ${ }^{1}$, G. Comi ${ }^{1}$, G . Magnani ${ }^{1}$

${ }^{1}$ San Raffaele Hospital, University Vita-Salute San Raffaele (Milano)

${ }^{2}$ University Vita-Salute San Raffaele (Milano)

${ }^{3}$ Giovanni XXIII Hospital, University Vita-Salute San Raffaele (Bergamo)

Objective: Correctly diagnosing Alzheimer's Disease (AD) in prodromal phases would allow to adopt new "disease modifying" drugs selectively targeting the pathogenetic process before neuronal damage gets irreversible. Therefore a great effort is being aimed at finding early reliable disease markers. Mild Cognitive Impairment (MCI) is an intermediate condition between cognitive changes of normal aging and initial phases of the real dementia. This study was aimed to identify a simple, cost effective and reliable diagnostic algorithm to predict conversion from MCI to AD.

Materials And Methods: We considered 96 consecutive MCI patients. At baseline all patients underwent neuropsychological assessment and lumbar puncture with CSF analysis of amyloid-B 42 (AB42), total tau (t-tau) and phosphorilated tau (ptau) levels. Each patient was followed up to identify a possible progression from $\mathrm{MCI}$ to $\mathrm{AD}$. An inde- 
pendent sample of $10 \mathrm{MCI}$ patients was considered to validate the cut off values of the predictors considered in the previous sample.

Results: A Composite Cognitive Score (CCS) was calculated including episodic memory, executive function and verbal fluency tests. Combining together CSF biomarkers and CCS increased the accuracy of the single predictors, correctly classifying $86 \%$ of patients with a specificity of $96 \%$ and a Positive Predictive Value of $93 \%$. CCS + CSF biomarkers correctly classify the majority of the MCI patients in the independent sample.

Discussion and Conclusions: CSF analysis and neuropsychological assessment can detect MCI patients who will convert to AD with high confidence. Their relative low cost and availability could make them worldwide essential tools in future clinical trials.

\section{References}

[1] Ferreira D. Meta-Review of CSF Core Biomarkers in Alzheimer's Disease: The State-of-the-Art after the New Revised Diagnostic Criteria. Front Aging Neurosci. (2014) Mar 24;6:47

[2] Ewers M. Prediction of conversion from mild cognitive impairment to Alzheimer's disease dementia based uponbiomarkers and neuropsychological test performance. Neurobiol Aging (2012) Jul;33(7):1203-14

[3] Tapiola T. Cerebrospinal fluid \{beta $\}$-amyloid 42 and tau proteins as biomarkers of Alzheimer-type pathologic changes in the brain. Arch Neurol. (2009) Mar;66(3):382-9

\section{EPIDEMIOLOGY}

\section{Screening for Mild Cognitive Impairment in Parkinson's Disease: comparison of the Italian Versions of Three Neuropsychological Tests}

\author{
A. Federico ${ }^{1}$, A. Maier ${ }^{1}$, G. Vianello², D. Mapelli², \\ M. Trentin ${ }^{3}$, G. Zanette ${ }^{3}$, A. Picelli ${ }^{4}$, M. Gandolfi, \\ S. Tamburin ${ }^{1}$ \\ ${ }^{1}$ Department of Neurological and Movement \\ Sciences, Neurology Section, University of Verona \\ (Verona) \\ ${ }^{2}$ Department of General Psychology, University of \\ Padova (Padova) \\ ${ }^{3}$ Section of Neurology, Pederzoli Hospital \\ (Peschiera del Garda-VR) \\ ${ }^{4}$ Neuromotor and Cognitive Rehabilitation \\ Research Centre, University of Verona (Verona)
}

Mild cognitive impairment (MCI) is frequent in Parkinson's disease (PD). Recently proposed criteria for $\mathrm{MCI}$ in $\mathrm{PD}$ (PD-MCI) indicate level I diagnosis based on abbreviated assessment and level II based on comprehensive neuropsychological evaluation. The study explored the sensitivity and specificity of the Italian versions of three neuropsychological tests for level I diagnosis of PD- MCI. We recruited 100 consecutive PD patients. After screening for inclusion criteria, 43 patients were included. The sensitivity and specificity of the Mini Mental State Examination (MMSE), the Montreal Cognitive Assessment (MoCA), and the Addenbrooke's Cognitive Examination Revised (ACE-R) in comparison to level II diagnosis of PD-MCI were examined. PDMCI was diagnosed (level II) in $51 \%$ of patients. Disease duration was significantly longer and PD motor scales were more severely impaired in MCI group. The receiver-operator characteristics curve documented nonsignificant difference in the performance of the three tests, with slight advantage of MMSE (corrected data). The time of administration favored MMSE. In Italian-speaking PD patients, MMSE might represent a good screening tool for PD-MCI, because of the shorter time of administration and the performance comparable to those of MoCA and ACE-R. Further studies are needed to validate the new PD-MCI criteria across different languages and cultures.

\section{Worldwide prevalence of dementia: the challenge of missing data}

\author{
S. Pomati ${ }^{1}$, A. Markova ${ }^{1}$, I. Cova ${ }^{1}$, I. Campini ${ }^{1}$, \\ G. Grande ${ }^{1}$, C. Mariani ${ }^{2}$ \\ ${ }^{1}$ Center for Research and Treatment on Cognitive \\ Dysfunctions, ASST Fatebenefratelli Sacco, "Luigi \\ Sacco" Hospital, University of Milan (Milano) \\ ${ }^{2}$ Department of Biomedical and Clinical Sciences \\ Luigi Sacco, University of Milan (Milano)
}

Whether an age- or an aging-related phenomenon, with increasing life expectancy dementia is steadily growing as a public health priority. A systematic review of the literature and meta-analysis conducted in 2013 [1] (with reference to studies published between 1980 and 2009, in 21 Global Burden of Disease regions) had estimated that 35.6 million people lived with dementia worldwide in 2010. The aim of our study was to emphasize the unavailability of information about dementia prevalence in several 
regions of the world. We performed a systematic review of the literature through PubMed (MeSH: "dementia" AND "epidemiology" OR "prevalence") and identified 124 relevant articles published between 1965 and 2015. Geo-economical distribution was evaluated through world bank membership (http://data.worldbank.org/country). Most studies were published in high and middle-high income countries (106) while only a small number of studies were conducted in low (4: Benin, Central African Republic) and middle-low income countries (14: Congo, Egypt, India, Nigeria, Sri Lanka). Overall scant and inadequate information was available for countries in Eastern Europe, Africa (Mediterranean and Sub Saharan) and Middle-East. Age-standardized prevalence was about $7.4 \%$ (interquartile range 4.8 - 10.9), with a nadir in India and a zenith in Brazil. Our results shows that in several regions of the world information about dementia is completely lacking: in addition to a call for adequate research in those settings, these data appear particularly challenging in view of the present migratory trends, given the significant implications for social and public policy and planning.

\section{Reference}

[1] Prince M, Bryce R, Albanese E, Wimo A, Ribeiro W, Ferri $\mathrm{CP}$. The global prevalence of dementia: A systematic review and metaanalysis. Alzheimers Dement. (2013);9:63-75

\section{Thrombophilia and Alzheimer's: is there a positive correlation?}

L. Iurato ${ }^{1}$, R. Grimaldi ${ }^{1}$, F. Caruso ${ }^{3}$, C. Occhipinti ${ }^{1}$, MG. Naso ${ }^{1}$, MG. Randisi ${ }^{1}$, J. Torrisi ${ }^{1}$,

LS. Verniccio ${ }^{1}$, MM. Vecchio ${ }^{2}$

${ }^{1}$ Neurologist Sant'Elia Hospital ASL Caltanissetta (Italy),

${ }^{2}$ Chief of Neurological Department Sant'Elia

Hospital ASL Caltanissetta (Italy)

${ }^{3}$ Neuropsychologist Sant'Elia Hospital ASL

Caltanissetta (Italy)

WE performed a project to evaluate if there is a positive association between thrombophilia and Alzheimer disease. Thrombophilia is an inherited (genetic) or acquired tendency to develop thrombosis. Thrombophilias can cause thrombosis by manufacturing too much clotting protein that are resistant to break down, producing too little of proteins that pre- vent thrombosis, or damaging the walls of the blood vessels. Thrombophilias can cause thrombosis in arteries, veins, or both. We selected 400 patients with Alzheimer's from our U.V.A Center. They are patients with Alzheimer age from 60 years to 80 in specific pharmacological therapy who have agreed to take a blood sample for testing for thrombophilia. We have to test the protein $\mathrm{C}$ deficiency, the protein $\mathrm{S}$ deficiency, Factor V Leiden, homocysteine, anticardiolipin antibodies, antiphospholipid antibodies, the LAC antibody. We also planned to carry out genetic testing for mutations and polymorphism in the prothrombotic gene and in the methylenetetrahydrofolate reductase gene. Our project began March 1, 2016 and will last six months. We have already selected a population of 400 healthy control elements, comparable in age to our population of patients with Alzheimer. Our project will help to clarify whether it is possible to assume a relationship between thrombophilia and Alzheimer's.

\section{References}

[1] Bots ML, Van Kooten F, Breteler MM, et al. Response to activated protein $\mathrm{C}$ in subjects with and without dementia. The Dutch Vascular Factors in Dementia Study. Haemostasis (1998) May-Aug; 28 (3-4): 209-15

[2] Chapman J, Wang N, Treves TA, Korczyn AD, Bornstein NM. ACE, MTHFR, Factor V Leiden and APO E polymorphism in patients with vascular and Alzheimer's dementia. Stroke( 1998) Jul; 29 (7): 1401-4

[3] Vaccarino L, Forte GI, Palmeri M et al. Role of prothrombotic polymorphism in successful or unsuccessful aging. Biogerontology (2011) Oct; 12(5):445-50

\section{GENETICS}

\section{Possible role of curcumins in RNA regulation in Alzheimer's disease patients}

S. Gagliardi ${ }^{1}$, S. Zucca ${ }^{1}$, S. Policicchio ${ }^{1}$, E. Sinforiani ${ }^{2}$, P. Bini ${ }^{3}$, J. Cashman ${ }^{4}$, C. Cereda ${ }^{1}$

${ }^{1}$ Genomic and Post Genomic Center,

“C. Mondino” National Neurological Institute (Pavia)

${ }^{2}$ Laboratory of Neuropsychology/UVA,

“C. Mondino” National Neurological Institute

(Pavia)

${ }^{3}$ Division of General Neurology, "C. Mondino”

National Neurological Institute (Pavia)

${ }^{4}$ Lab. Chemistry, Human Biomolecular Research

Institute (San Diego) 
Several strategies have been studied to improve the immune system in AD. The natural product mixture of curcuminoids, that improve certain defects in innate immune cells of AD patients may selectively enhance Abeta phagocytosis, attenuate APP maturation and alter gene transcription (Fiala et al., 2007; Gagliardi et al., 2012). Different compound have been tested and one of the most potent was bisdesmethoxycurcumin (BDC). We have tested five different synthetic curcumins, BDC, 150, 085, sg-117 end sg-180 on PBMCs from AD and controls and we have evaluated genetic expression via NGS to identify the molecular pathways target of curcumin action. The comparison between patients and controls has revealed a significant alteration of the expression in 12 genes of more than 100 genes tested, NFKB and BACE1 appear to be up-regulated in patients than in controls while MGAT3 and VDR are subject to a down-regulation. NGS analysis has revealed that only 3 on the 5 tested curcumins appear to be active, these are BDC, SG-150 and SG-085 confirming the protective action of curcumins towards treatment with $A \beta$. The reduced levels of NFKB may result in a decrease of the inflammatory cascade. This decline could be due to a direct effect of curcumin on NFKB pathway or a consequence of the increased levels of VDR which has an inhibitory action on NFKB through IKKB factors (Wang et al., 2012). Therefore, the preliminary data suggest a proof of concept for a future pharmacological intervention using curcumins as dietary supplement in asymptomatic $\mathrm{AD}$ patients.

\section{References}

[1] Fiala M, Liu PT, Espinosa-Jeffrey A, Rosenthal MJ, Bernard G, Ringman JM, Sayre J, Zhang L, Zaghi J, Dejbakhsh S, Chiang B, Hui J, Mahanian M, Baghaee A, Hong P, Cashman J. Innate immunity and transcription of MGAT-III and Toll-like receptors in Alzheimer's disease patients are improved by bisdemethoxycurcumin. Proc Natl Acad Sci U S A. (2007); 104(31):12849-54

[2] Gagliardi S, Ghirmai S, Abel KJ, Lanier M, Gardai SJ, Lee C, Cashman JR. Evaluation in vitro of synthetic curcumins as agents promoting monocytic gene expression related to $\beta$-amyloid clearance. Chem Res Toxicol. (2012);25(1):10112

[3] Wang L, Hara K, Van Baaren JM, Price JC, Beecham GW, Gallins PJ, Whitehead PL, Wang G, Lu C, Slifer MA, Züchner S, Martin ER, Mash D, Haines JL, Pericak-Vance MA, Gilbert JR. Vitamin D receptor and Alzheimer's disease: a genetic and functional study. Neurobiol Aging. (2012);33(8):1844. e1-9

\section{Type 2 diabetes and Alzheimer's disease: evidences for a shared genetic etiology}

\author{
P. Mazzola ${ }^{1}$, L. $\mathrm{Ho}^{2}$, K. $\mathrm{Hao}^{3}$, G. Pasinetti ${ }^{2}$ \\ ${ }^{1}$ Department of Medicine and Surgery, University \\ of Milano-Bicocca (Monza) \\ ${ }^{2}$ Department of Neurology, Icahn School of \\ Medicine at Mount Sinai (New York) \\ ${ }^{3}$ Department of Genomics, Icahn School of \\ Medicine at Mount Sinai (New York)
}

Epidemiological evidence supports the observation that subjects with type 2 diabetes (T2D) are at higher risk to develop Alzheimer's disease (AD). However, how these conditions are causally linked and how dietary lifestyles interact with these conditions is unknown. Possible mechanisms include shared genetic risk factors, which we investigated in a recent study based on recent genome wide association study (GWAS) findings. We retrieved single nucleotide polymorphisms (SNPs) associated with $\mathrm{T} 2 \mathrm{D}$ and $\mathrm{AD}$ from large-scale GWAS meta-analysis consortia and tested for overlap among the T2D and AD associated SNPs. We found 927 SNPs associated with both $\mathrm{AD}$ and T2D ( $\mathrm{p}$-value $\leq 0.01$ ), an overlap significantly larger than random chance (overlapping p-value of 6.93E-28). Among these, 395 of the shared GWAS SNPs have the same risk allele for $\mathrm{AD}$ and $\mathrm{T} 2 \mathrm{D}$, suggesting common pathogenic mechanisms underlying their development. We found that gene annotations from these shared SNPs are significantly enriched for specific KEGG pathways pertaining to immune responses, cell signaling and neuronal plasticity, whose abnormalities are known to contribute to both T2D and AD pathogenesis. This suggests that among T2D subjects with common genetic predispositions, dysregulation of these pathogenic pathways could have contributed to the onset of T2D, while simultaneously contributing to their increased risk of developing AD. Collectively, our GWAS studies tentatively support the epidemiological observation of disease concordance between T2D and AD. Ongoing studies are investigating whether intensive lifestyle interventions in T2D subjects with genetic predisposition to AD may concurrently reduce T2D phenotypes and/or attenuate $\mathrm{AD}$ onset and progression.

\section{Reference}

[1] Wang J, Gong B, Zhao W, Tang C, Varghese M, Nguyen T, Bi W, Bilski A, Begum S, Vempati P, Knable L, Ho L, Pasinetti GM. Epigenetic mechanisms linking diabetes and synaptic impairments. Diabetes. (2014);63(2):645-54. doi: 10.2337/ db13-1063 


\section{A novel PSEN1 mutation in a family with late-onset Alzheimer's disease}

\author{
I. Ricca ${ }^{1}$, M. Valente ${ }^{1}$, G.S. Grieco ${ }^{1}$, P. Bini ${ }^{2}$, \\ P. Vitali ${ }^{3}$, C. Cereda ${ }^{1}$ \\ ${ }^{1}$ Center of Genomics and post-Genomics, \\ C. Mondino National Institute of Neurology \\ Foundation (Pavia) \\ ${ }^{2}$ Neurological Department, C. Mondino National \\ Institute of Neurology Foundation (Pavia) \\ ${ }^{3}$ Neuroradiology and Brain MRI Mondino Research \\ Center, C. Mondino National Institute of Neurology \\ Foundation (Pavia)
}

Objectives: PSEN1 mutations are reported mainly in early-onset Alzheimer's disease (EOAD), frequently associated with other neurological features, including seizures, spastic paraparesis, myoclonus and cerebellar signs. Here we report two sisters suffering from late-onset Alzheimer's disease, carrying a novel mutation in the PSEN1 gene.

Materials and methods: The two patients were born from healthy nonconsanguineous parents. They both presented late-onset memory deficits, abulia and episodes of confusion without other associated neurological disturbances. They both showed a similar peculiar cerebral MRI pattern characterized by bilateral asymmetric global atrophy, marked in the temporomesial areas, with a prominent involvement of the left hemisphere. The parents died both after 85 years of age and they did not showed any AD symptom. The genomic DNA of the two sisters was extracted according to standard protocols. All the PSEN1, APP and PSEN2 exons were amplified and automatically sequenced (Sanger sequencing).

Results: We found a novel heterozygous variant in exon 4 of the PSEN1 gene (c.134G>A/p.G45V; NM_00021/NP_000012). This variant in not reported in the 1000GS and ExAC databases and the prediction tools (Polyphen II and Mutation Taster) indicate this mutation is likely to have a pathological effect on protein function. DNA sample of the parents were not available for segregation studies.

Discussion and conclusions: We suggest that the c.134G $>$ A/p.G45V variant will have probably a pathogenic effect, hypothesizing a possible role in late onset $\mathrm{AD}$.

\section{NEUROBIOLOGY}

\section{HSV-1 avidity index: a biomarker for monitoring the evolution of Mild Cognitive Impairment to Alzheimer's disease?}

S. Agostini ${ }^{1}$, R. Mancuso ${ }^{1}$, F. Baglio ${ }^{2}$, M. Cabinio ${ }^{2}$, A. Hernis ${ }^{1}$, F.R. Guerini ${ }^{1}$, E. Calabrese ${ }^{3}$, R. Nemni ${ }^{4}$, M. Clerici ${ }^{4}$

${ }^{1}$ Laboratory of Molecular Medicine and Biotech,

Don C. Gnocchi Foundation IRCCS - ONLUS

(Milano, Italy)

${ }^{2}$ MRI Laboratory, Don C. Gnocchi Foundation

IRCCS - ONLUS (Milano, Italy)

${ }^{3}$ Department of Neurorehabilitation, Don C.

Gnocchi Foundation IRCCS - ONLUS (Milano,

Italy)

${ }^{4}$ Department of Pathophysiology and

Transplantation, University of Milano (Milano, Italy)

Objectives: Mild Cognitive Impairment (MCI) is a condition characterized by mild changes in thinking abilities that exceed those observed in the physiological age-associated cognitive decline. MCI is often prodromic of the development of Alzheimer's disease (AD). A viral hypothesis is considered for $\mathrm{AD}$; HSV-1, in particular, has been suggested to associate with this condition. We analyzed whether immune responses to HSV-1 play a role in AD conversion by evaluating the IgG HSV-1-specific humoral response of MCI individuals.

Materials: 80 individuals with a diagnosis of MCI were enrolled. Serum samples were collected at baseline and a randomly selected subgroup underwent brain magnetic resonance imaging (MRI) by 1.5T scanner. All individuals were clinically reevaluated after 24 months. Antibody $(\mathrm{Ab})$ titers against HSV-1 and Ab avidity were measured by ELISA.

Results: At the 24 months' time point AD conversion was detected in 21 individuals (MCI-converter); results were compared to those of 15 subjects in whom $\mathrm{AD}$ conversion was not observed (MCI-nonconverter). Whereas no significant differences were observed in HSV-1-specific Ab titers, the avidity of HSV-1-specific Ab was significantly higher in MCInon-converter (median: 100; IQR:93.53-100\%) compared to MCI-converter (89.09; 82.74-94.32\%; $\mathrm{p}=0.0018$ ). Notably, a positive correlation between HSV-1 Ab titers and cortical volume in the left 
hippocampus and amigdala, as evaluated by MRI $(\mathrm{p}<0.05)$, was detected.

Conclusions: Stronger HSV-1-specific humoral responses are associated with protection against $\mathrm{AD}$ conversion and better preserved cortical volumes. These results reinforce the hypothesis of a role for HSV-1 in the pathogenesis of AD.

\section{NMR-based identification of anti- Alzheimer's compounds in edible plants and foods}

C. Airoldi ${ }^{1}$, C. Ciaramelli ${ }^{1}$, A. Palmioli ${ }^{1}$, V. Mazzoni ${ }^{1}$, R. Bussei ${ }^{1}$, L. Colombo ${ }^{2}$,

A. De Luigi ${ }^{2}$, F. Nicotra ${ }^{1}$

${ }^{1}$ Department of Biotechnology and Biosciences, University of Milano-Bicocca (Milano)

${ }^{2}$ Department of Molecular Biochemistry and Pharmacology, Mario Negri Institute (Milano)

Objectives: There is an urgent need for new strategies for Alzheimer's disease (AD) early diagnosis and treatment, as well as for its prevention. Diet could be a very effective tool. Aim of our work is the development of a new NMR-based approach allowing the screening of extracts from edible plant and food matrixes aimed at the identification of anti-Alzheimer's compounds.

Materials: Salvia sclareoides, Genista tenera and green tea leafs were collected in nature or from commercial sources. Coffee beans and hop pellets were provided respectively from a coffee producer and a brewer. $A \beta$ peptide was prepared by solid-phase peptide synthesis.

Method: Extracts were obtained according to different procedures involving water and/or organic solvent extractions. The metabolic profiles of the extracts and the molecular interaction with $\mathrm{A} \beta$ oligomers were characterized by NMR spectroscopy.

Results and Discussion: After their 1H-NMR metabolic profiling, we identified $A \beta$ oligomer ligands in salvia [1], Genista [2], green tea [3], coffee and hop extracts by exploiting STD NMR and trNOESY experiments. Moreover, their antioxidant and antiamyloidogenic activities were evaluated by cellular and biochemical assays, supporting the correlation among the recognition of the molecular targets and the biological responses.

Conclusions: Our data provide an effective method to identify new anti-AD compounds and to gain fundamental information for the comprehension of the biological and nutraceutical activities exerted by foods within the context of AD.

\section{References}

[1] C. Airoldi, E. Sironi, C. Dias, F. Marcelo, A. Martins, A. P. Rauter, F. Nicotra, J. Jimenez-Barbero. Natural compounds against Alzheimer's disease: molecular recognition of A $\beta 1$ 42 peptide by Salvia sclareoides extract and its major component, rosmarinic acid, as investigated by NMR. Chem. Asian J. (2013);8:596-602

[2] Jesus A. R., Dias C., Matos A. M., de Almeida R. F. M., Viana A. S., Marcelo F., Ribeiro R. T., Macedo M. P., Airoldi C., Nicotra F., Martins A., Cabrita E. J., Jiménez-Barbero J., Pilar Rauter A., J. 8- $\beta$-D-Glucopyranosylgenistein: Synthesis, Antidiabetic Activity and Molecular Interaction with Islet Amyloid Polypeptide and Amyloid $\beta$-Peptide (1-42). Med. Chem. (2014);57:9463-9472

[3] Sironi E., Colombo L., Lompo A., Messa M., Bonanomi M., Regonesi M. E., Salmona M., Airoldi C., Natural Compounds against Neurodegenerative Diseases: Molecular Characterization of the Interaction of Catechins from Green Tea with A $\beta 1-42$, PrP106-126, and Ataxin-3 Oligomers. Chem. Eur. J. (2014);20:13793-13800

\section{Aß, LPS and their affinity for cell membrane lipid bilayers}

\author{
A. Asti ${ }^{1}$, S. Saccucci ${ }^{1}$, N. Marchesi ${ }^{3}$, A. Pascale ${ }^{3}$, \\ F. Baggi ${ }^{2}$, A. Consonni ${ }^{2}$, R. Mantegazza ${ }^{2}$ \\ ${ }^{1}$ Neuropathology and Neurology V, IRCCS \\ Foundation Neurological Institute "C. Besta" \\ (Milano) \\ ${ }^{2}$ Neuroimmunology and Neuromuscolar diseases, \\ IRCCS Foundation Neurological Institute \\ "C. Besta" (Milano) \\ ${ }^{3}$ Pharmacological Science Department, University \\ of Pavia (Pavia)
}

Objective: $\mathrm{A} ß$ belongs to Antimicrobial Peptides (AMPs), broad spectrum bactericides targeting Gram- and Gram + bacteria; the objective is to show that $A \beta 25-35, A \beta 1-42$ alone/or with E. coli LPS have affinity for lipid bilayers and that factors such as hydrophobicity and amphipathicity are involved.

Materials and Methods: A $\beta 25-35, \mathrm{~A} \beta 1-42, \mathrm{LPS}$, E. coli serotype 0128:B12, were dissolved separately in distilled water, final concentration $10 \mu \mathrm{g} / \mathrm{ml}$, $1 \mu \mathrm{g} / \mathrm{ml}$ for LPS. Cell culture and treatment:

$1 \times 106$ cells $/ \mathrm{mL}$ human neuroblastoma (SHSY5Y) grown in Eagle's medium with $10 \%$ fetal calf serum, $1 \%$ penicillin-streptomycin, L-glutamine $(2 \mathrm{mM})$, nonessential amino acids $(1 \mathrm{mM})$, sodium pyruvate $(1 \mathrm{mM})$.

- SH-SY5Y untreated
- SH-SY5Y/ LPS
- SH-SY5Y/A $\beta 25-35$ 
- SH-SY5Y/A $\beta$ 1-42

- SH-SY5Y/LPS/A $\beta 25-35$

- SH-SY5Y/LPS/A $\beta$ 1-42

- each experiment at $37^{\circ} \mathrm{C}$, for $24,48,72$ hours.

\section{Cytokine quantification:}

TNF- $\alpha$, IL-1 $\beta$, IL-6, IL-8 IFN- $\gamma$ has been assessed by ELISA.

Transmission electron microscopy (TEM) by standard technique

Results: Cytokine quantification show pro-inflammatory profiles with enhanced TNF- $\alpha$, IL- 8, IFN- $\gamma$ levels TEM: $A \beta$ and LPS show affinity for lipid bilayers and exert activity by membrane permeabilization assessed by cytokine production. At 72 hours the synergistic action of $A \beta$ and LPS promotes mitochondrial alteration; IL-1beta, IL-6 and IL-8 have higher values

Discussion: Cell membrane stain positive for $\mathrm{A} \beta$ and LPS; this indicates an affinity at the molecular level and induce intracellular calcium deregulation by membrane permeabilization that leads to apoptosis through mitochondrial dysfunction, by direct or indirect association with the cell membrane.

Conclusions: Aß, LPS are involved in the regulation of inflammation by activating chemokine release; an infectious origin might give an explanation of the cellular, molecular, inflammatory processes involved in neurodegenerative diseases.

\section{References}

[1] Soscia S et al. The Alzheimer's Disease-Associated Amyloid b-Protein Is an Antimicrobial Peptide. PlosOne (2010); 5:110

[2] Rosenfeld Y et al. Endotoxin (Lipopolysaccharide) Neutralization by Innate Immunity Host-Defense Peptides. J of Biol Chem. (2006);281:1636-1643

[3] Asti A et al. Can a bacteruial endotoxin be a key factor in the kinetic of amyloid fibril formation? JAD (2014);39(1):169-79

\section{Alzheimer's Disease CSF biomarkers are significantly reduced in patients with Normal Pressure Hydrocephalus: what does it mean?}

G. Cecchetti ${ }^{1}$, R. Santangelo ${ }^{1}$, S. Mazzeo ${ }^{1}$, P. Pinto ${ }^{2}$, G. Passerini ${ }^{1}$, R. Cardamone ${ }^{1}$, A. Franzin ${ }^{1}$, G. Comi ${ }^{1}$, G. Magnani ${ }^{1}$

${ }^{1}$ San Raffaele Hospital, University Vita-Salute San Raffaele (Milano)

${ }^{2}$ Papa Giovanni XXIII Hospital (Bergamo)
Objectives: The aim of this study was to evaluate the existence of a typical CSF profile in NPH that could differentiate it from $\mathrm{AD}$ and other kinds of dementia and to preliminarily investigate the amyloid role in NPH.

Material and Methods: We analyzed the concentration of CSF AD biomarkers (A $\beta 42$; tau and p-tau) in a sample of 344 subjects: 170 with Alzheimer's Disease (AD), 34 with Normal Pressure Hydrocephalus (NPH), 106 with other types of dementia and 34 with a not neurodegenerative disorder (CONTROL)

Results: There are no significant differences in the total amount of CSF proteins in all the groups considered. The mean concentrations of $A \beta 42$ in $A D$ and NPH are significantly lower than in controls, while there are no differences between $\mathrm{AD}$ and NPH. Moreover in AD and NPH the number of patients with pathological $A \beta 42$ levels is significantly higher than in controls and patients with other type of dementia. Tau and p-tau concentrations are significantly lower in NPH subjects than in AD and other dementias, while there are no differences between $\mathrm{NPH}$ and controls.

Discussion and Conclusion: $\mathrm{A} \beta 42$, tau and p-tau mean concentrations are significantly reduced in NPH patients. This cannot be due to a simple dilution effect, since the CSF protein content do not differ between NPH and the other groups. This could indicate a possible accumulation of beta amyloid in NPH subjects. On the other hand, the reduced tau and p-tau mean concentrations in NPH, similar to controls, might suggest the absence of a marked neuronal damage.

\section{References}

[1] Tae SL, Jun YC, Sun AP, Young CY, Hyun YL, Byung GK, In SJ, Kyoon $\mathrm{H}$ and So YM, Evaluation of coexistence of Alzheimer's disease in idiopathic normal pressure hydrocephalus using ELISA analyses for CSF biomarkers, BMC Neurology (2014);14:66

[2] GD Silverberg, M Mayo, T Saul, E Rubenstein, and D McGuire, Alzheimer's disease, normal-pressure hydrocephalus, and senescent changes in CSF circulatory physiology: a hypothesis, THE LANCET Neurology (2003); 2:506-511

[3] GD Silverberg, MC Millera, JT Machanb, CE Johansona, IN Caralopoulosa, CL Pascalea, A Heilec, PM Klingea, Amyloid and Tau accumulate in the brains of aged hydrocephalic rats, Brain Research (2010);1317:286-96 


\section{Peripheral sink effect as possible nanotherapeutic strategy for} Alzheimer's disease: an ex vivo study

\author{
E. Conti ${ }^{1}$, C. Zoia ${ }^{1}$, D. Grana ${ }^{1}$, F. Da Re ${ }^{2}$, \\ M. Gregori ${ }^{3}$, F. Re ${ }^{3}$, M. Masserini ${ }^{3}$, C. Ferrarese ${ }^{2}$, \\ L. Tremolizzo ${ }^{2}$ \\ ${ }^{1}$ Laboratory of Neurobiology, School of Medicine \\ and Surgery and NeuroMi, University of Milano- \\ Bicocca (Monza) \\ ${ }^{2}$ Laboratory of Neurobiology, School of Medicine \\ and Surgery, NeuroMi and San Gerardo \\ Hospitalience and Department of Neurology, \\ University of Milano-Bicocca and San Gerardo \\ Hospital (Monza) \\ ${ }^{3}$ School of Medicine and Surgery and \\ Nanomedicine Center NANOMIB, University of \\ Milano-Bicocca (Monza)
}

Objectives: The accumulation of extracellular amyloid beta (Abeta42) both in brain and in cerebral vessel characterizes Alzheimer's disease (AD) pathogenesis. Given the existing equilibrium of Abeta42 across the blood brain barrier, the reduction of central Abeta42 may be achieved through the so called "peripheral sink effect". The possibility to functionalize nanoparticles (NPs) surface with Abeta42 binding molecules has been shown effective in Abeta42 reduction in AD models. Here we tested the capacity of functionalized liposomes to sequester Abeta42 in human biological fluids, both plasma and cerebro-spinal fluid (CSF).

Methods: Liposomes functionalized with phosphatidic acid (PA), and a modified Apolipoprotein E-derived peptide (mApo), or with a curcuminederivative (TREG), were used. Plasma or CSF samples were incubated $\left(10 \mathrm{~min}\right.$ at $\left.37^{\circ} \mathrm{C}\right)$ with liposomes, than Abeta42 levels were determined by ELISA.

Results: mApo-PA $(250 \mu \mathrm{M})$ significantly reduced Abeta42 levels in CSF samples from healthy subjects $(\mathrm{p}<0.01)$. Analogously, both PA or TREG $(25$ $\mu \mathrm{M})$ were able to sequester highly concentrated (2.5 $\mu \mathrm{M})$ Abeta42 exogenously added to AD plasma samples. Finally, a significant reduction $(\sim 20 \%)$ in Abeta42 content $(p<0.001)$ was demonstrated following exposure to all functionalized liposomes in plasma samples from selected AD or Down's syndrome patients expressing high levels of Abeta42. The same results were obtained by quantifying Abeta content after removal of liposomes bound Abeta by using gel filtration chromatography.
Conclusion: Our data report the capacity of functionalized liposomes to sequester Abeta in human biological fluids. This data may be critical for future in vivo administration tests using NPs for promoting sink effect.

\section{Early alterations in the expression of antioxidant enzymes and oxidative stress markers in neurogenic niches of TG2576 mouse model of Alzheimer's disease}

\author{
A. Fracassi ${ }^{1}$, F. Colasuonno ${ }^{1}$, G. Taglialatela ${ }^{2}$, \\ S. Moreno ${ }^{1}$ \\ ${ }^{1}$ Dept. of Science, University Roma Tre (Roma) \\ ${ }^{2}$ Dept. of Neurology, University of Texas Medical \\ Branch (Galveston-USA)
}

Adult neurogenesis, a multi-step process consisting in the continuous generation of new neurons, occurs in two specific brain regions (neurogenic niches), namely subventricular zone (SVZ) of the lateral ventricle and the subgranular zone (SVG) of the dentate gyrus of the hippocampus [1]. We here addressed the oxidative stress status and the elicited cellular response in the neurogenic niches at the onset of Alzheimer's disease (AD). As a suitable model for this purpose we chose the $\mathrm{Tg} 2576(\mathrm{Tg})$ mouse strain [2] and we focused on SGZ and SVZ of 3 month-old mice to study oxidative damage markers and antioxidant enzymes levels. Alterations in the antioxidant response in Tg compared to wild type (WT) were demonstrated by immunohistochemical analyses. Particularly, the expression of the peroxisomal enzyme catalase appears to be increased in both SGZ and SVZ. These data are consistent with the increase of PPARalpha levels in Tg, suggesting that early variations might represent a way to counteract the redox imbalance appearing at the early stages of the pathology. Indeed early oxidative stress status was confirmed by the occurrence of nucleic acid oxidative damage in both areas showed by the higher 8-OHG immunoreactivity in Tg than in WT. Conversely SOD1 and SOD2 levels were decreased in Tg SGZ and SVZ as well as GPx levels. In overall agreement with our previous work on the neocortex [3], our observations support the concept that oxidative stress is a primary culprit in $\mathrm{AD}$ and the importance of molecular changes at early stages of the pathology. 


\section{References}

[1] Zhao C, Deng W, Gage FH. Mechanisms and functional implications of adult neurogenesis. Cell. (2008) Feb 22;132(4):645-60

[2] Hsiao K, Chapman P, Nilsen S, Eckman C, Harigaya Y, Younkin S, Yang F, Cole G. Correlative memory deficits, Abeta elevation, and amyloid plaques in transgenic mice. Science. (1996) Oct 4;274(5284):99-102

[3] Porcellotti S, Fanelli F, Fracassi A, Sepe S, Cecconi F, Bernardi $\mathrm{C}$, Cimini $\mathrm{A}$, Cerù MP, Moreno $\mathrm{S}$. Oxidative Stress during the Progression of $\beta$-Amyloid Pathology in the Neocortex of the Tg2576 Mouse Model of Alzheimer's Disease. Oxid Med Cell Longev. (2015);2015:967203

\section{Behavioural anomalies in neurodegenerative diseases: role of the immune response}

D. Grana ${ }^{1}$, E. Conti ${ }^{1}$, C. P. Zoia ${ }^{1}$, L. Maggioni ${ }^{2}$, A. Arosio' ${ }^{1}$, G. Stefanoni ${ }^{3}$, A. Aliprandi ${ }^{4}$, I. Appollonio ${ }^{1}$, C. Ferrarese ${ }^{1}$, L. Tremolizzo ${ }^{1}$ ${ }^{1}$ School of Medicine and Surgery, Milan Center for Neuroscience (NeuroMI), University of MilanoBicocca (Monza)

${ }^{2}$ School of Medicine and Surgery, University of Milano-Bicocca (Monza)

${ }^{3}$ School of Medicine and Surgery, Neurology "San Gerardo" Hospital (Monza)

${ }^{4}$ Neurology Unit, Manzoni Hospital (Lecco)

Objectives: The main cause of distress in caregivers of Alzheimer's Disease (AD) patients is represented by Behavioural and Psychological Symptoms of Dementia (BPSD), which biological bases are still unknown. Translocator protein $18 \mathrm{kDa}$ (TSPO) is a biomarker of neuroinflammation and regulates monocyte chemotaxis and blood-brain barrier crossing. The TSPO gene contains the rs6971 SNP that has been associated to separation anxiety and influences PK11195 PET binding. The aim of this work consisted in studying TSPO as a potential target for understanding BPSD.

Methods: 41 probable AD patients underwent to the NeuroPsychiatric Inventory (NPI). DNA was genotyped and we semi-quantified TSPO mRNA by RT-PCR and DBI (TSPO endogenous ligand) by ELISA. A chemotaxis assay was developed using THP-1 cells and MCP-1/Abeta42 as chemoattractants.

Results: The distribution of the rs6971 SNP was: $12 \%$ AA, 37\% AG, 51\% GG. No significant differences were found in TSPO mRNA levels neither comparing AD to controls nor SNP subgroups. DBI was increased by $65 \%$ in $\mathrm{AD}$ versus CTRL. No association was found between NPI scores and bio- logical parameters. As concerns chemotaxis, THP-1 cells showed a significant increase of the Forward Migration Index with both MCP-1 and Abeta42.

Discussion: At the moment, no association was found between the investigated parameters and BPSD. Abeta42 shows clear chemoattractant properties that need further investigation.

Conclusions: Before concluding on a potential role of TSPO in AD-related BPSD pathogenesis, we suggest that we should increase sample size. DBI role deserves further investigation as well, especially considering the well-known GABA-A receptor inverse agonist properties.

\section{Protective action of fingolimod against acute $A \beta$ neurotoxicity by rapid synaptic relocation of NMDA receptor in hippocampal neurons}

P. Joshi ${ }^{1}$, M. Gabrielli ${ }^{2}$, L. Ponzoni ${ }^{3}$, S. Pelucchi ${ }^{4}$, M. Stravalaci ${ }^{5}$, M. Beeg ${ }^{5}$, S. Mazzitelli ${ }^{1}$, B. Braida ${ }^{3}$, M. Sala ${ }^{3}$, E. Boda ${ }^{6}$, A. Buffo ${ }^{6}$, M. Gobbi ${ }^{5}$, F. Gardoni ${ }^{4}$, M. Matteoli ${ }^{1}$, E. Marcello ${ }^{4}$, C. Verderio ${ }^{2}$

${ }^{1}$ Department of Neuroscience, IRCCS Humanitas (Rozzano-MI)

${ }^{2}$ Department of Neuroscience, IN-CNR (Milano) ${ }^{3}$ Department of Biotechnology and Translational Medicine, University of Milano (Milano) ${ }^{4}$ Department of Pharmacological and Biomolecular Sciences, University of Milan (Milano)

${ }^{5}$ Department of Molecular Biochemistry and Pharmacology, IRCSS Istituto di Ricerche Farmacologiche Mario Negri (Milano) ${ }^{6}$ Department of Neuroscience Rita Levi-Montalcini, University of Turin (Torino)

Objective: To investigate molecular mechanism responsible for direct preventive action of Fingolimod (FTY720) in cultured hippocampal neurons against acute neurotoxicity induced by toxic $A \beta$ 1-42 forms.

Materials: Cultured hippocampal neurons, Transgenic APPPS1 mice, soluble A $\beta$ 1-42 (s-A $\beta$ ), Fura 2/ am, S1P-R1 and S1P-R3 selective antagonists W146 and CAY 10444 respectively, SEP-GluN2A GluN2B constructs.

Methods: Single cell calcium imaging, Live imaging, Surface Plasma Resonance, Electrophysiology, Immunostaining and Western blotting.

Results and Discussion: Fingolimod, is an analogue of spingolipid sphingosine, which has been proved to be neuroprotective in rodent models of 
Alzheimer's disease (AD). Several mechanisms underlie neuroprotective action of FTY. In this study we show that $1 \mathrm{~h}$ pre-incubation of cultured neurons with FTY protects from the abnormal elevations of cytosolic calcium induced by 1 microM s-A $\beta$. Pretreatment of neurons $1 \mathrm{~h}$ with FTY decreases $30 \%$ of NMDA-mediated calcium transient evoked by s-A $\beta$ or NMDA. The decrease in NMDA-mediated calcium influx is prevented by antagonists of S1P receptors, revealing involvement of SIP pathway. Isolation of synaptic and extrasynaptic NMDA-mediated calcium responses along with analysis of miniature spontaneous NMDA postsynaptic currents shows that FTY largely decreases responsiveness of extrasynaptic receptors, whereas responsiveness of synaptic NMDA receptors increases significantly. Biochemical assays reveal that FTY induces a rapid increase in GLUN2A-containing neuroprotective NMDARs on the surface of postsynaptic spines, whereas mobilizes extrasynaptic GLUN2B-containing NMDARs, which are coupled to cell death, to the synapses.

Conclusion: The present findings suggest that rapid synaptic relocation of NMDARs might have protective impact against neurotoxicity induced by s- $\mathrm{A} \beta$.

\section{Increased TLR8 and 9 expression correlates with preserved hippocampal volumes and lack of progression to $\mathrm{AD}$ in Mild Cognitive Impairment individuals}

\author{
F. La Rosa ${ }^{1}$, M. Saresella ${ }^{1}$, F. Piancone ${ }^{1}$, \\ I. Marventano ${ }^{1}$, E. Calabrese ${ }^{2}$, R. Nemni², \\ F. Baglio ${ }^{3}$, M. Cabinio ${ }^{3}$, M. Clerici ${ }^{4}$ \\ ${ }^{1}$ Laboratory of Molecular Medicine and Biotech, \\ Don Carlo Gnocchi Foundation IRCCS-ONLUS \\ (Milano) \\ ${ }^{2}$ Department of Neurorehabilitation, Don Carlo \\ Gnocchi Foundation IRCCS-ONLUS (Milano) \\ ${ }^{3}$ MRI Laboratory, Don Carlo Gnocchi Foundation \\ IRCCS-ONLUS (Milano) \\ ${ }^{4}$ Department of Pathophysiology and \\ Transplantation, University of Milano (Milano)
}

Aim: A percentage of individuals with a diagnosis of (MCI) progresses to Alzheimer's disease (AD); we examined TLR expression and cytokine production in MCI individuals who did or did not progress to $\mathrm{AD}$ over a 24 -months time period.
Methods: Longitudinal study enrolling 46 individuals with a diagnosis of MCI who, did or did not progress to AD. TLRs expression (upon TLRs agonists-stimulation) and Ab1-42 stimulated cytokine production were measured by Flow Cytometry. ApoE4 status and hippocampus left and right volumes (HV) (using NMR techniques) were analyzed.

Results: MCI individuals who subsequently non progressed to AD: 1) TLR8 and TLR9 expression on CD14+ cells was significantly up-regulated $(\mathrm{p}<0.05) ; 2)$ Ab1-42-stimulated production of the pro-inflammatory cytokines IL6, TNFa, IL1b by CD14+ cells was significantly reduced $(p<0.05)$; and 3) left and right HV were better preserved $(\mathrm{p}<0.05)$. No differences were noticed in ApoE.

Discussion: Correlates predicting such progression are currently unknown. Because:

1) microorganisms, and in particular herpes simplex virus (HSV), are suggested to be involved in the pathogenesis of $\mathrm{AD}$. 2) innate immune responses to pathogens are triggered by the ligation of toll like receptors (TLR). 3) the expression of these receptors was show to be significantly up-regulated in MCI compared to AD individuals, suggesting that stronger innate immune responses are present in MCI.

Conclusions: TLR8 and TLR9 expression as well as the production of proinflammatory cytokines correlates with better preserved $\mathrm{HV}$ in MCI individuals who do not progress to $\mathrm{AD}$, suggesting that the ability to mount stronger antiviral responses associates with protection against $\mathrm{AD}$.

\section{Ischemic injury elicits the unconventional secretion of protein hallmarks of Alzheimer's disease onset as seeds for interneuronal propagation}
E. Lonati ${ }^{1}$, G. Sala ${ }^{1}$, V. Tresoldi ${ }^{2}$, S. Coco ${ }^{1}$, C. Milani ${ }^{1}$, A. Restelli ${ }^{2}$, F. Farina ${ }^{1}$, L. Botto ${ }^{1}$, D. Salerno ${ }^{2}$, P. Palestini ${ }^{1}$, A. Bulbarelli ${ }^{1}$
${ }^{1}$ Department of Medicine and Surgery, Milan Center for Neuroscience, University of Milano- Bicocca (Monza)
${ }^{2}$ Department of Medicine and Surgery, University of Milano-Bicocca (Monza)

Objectives: Understanding the ischemic injury contribution to Alzheimer's disease (AD) onset, pinpointing to post-translational modifications, turnover alterations, and secretion of proteins and networks identified as AD hallmarks. 
Materials: Neuronal cultures prepared from hippocampi of E18-E19 embryos from pregnant Sprague Dawley rats. 5\%CO2: 95\%N2 gaseous mixtures (Sapio); hypoxia chamber (BillupsRothenberg).

Methods: DIV8 neurons were subjected to oxygen and glucose deprivation (OGD). After medium replacement with a glucose-free balanced salt solution (ogR), cells were incubated for 3 hours in the chamber saturated with the gaseous mixture. The restoration solution (glucose and B27) was administrated to neurons (ogR) for one hour (R1h) or overnight (R16h).

Results: We observed Tau and amyloid precursor protein (APP) post-translational and protein level modifications after OGD/ogR. Tau cleavage and secretion occurs at R1h through microvesicles (MVs) population, including LC3 and/or LAMP1 positive vesicles, marker of autophagy-mediated secretion (exophagy). In MVs extract we also identified $\alpha$ - and $\beta$-C-terminal fragments (CTFs) of APP, and the peptidyl prolyl cis/trans isomerase Pin1.

Discussion: MVs represent an intercellular communication delivering multiple cargo with beneficial or harmful messages [1]. Thus, differences in MVs contents after OGD/ogR suggest that ischemia leads to a robust upheaval with the autophagic mechanisms activation resultant in neuron-to-neuron transfer of material with neurodegenerative potential.

Conclusions: OGD treatment leads to mis-processed protein unconventional secretion also including exophagy pathway. These proteins represent seeds for protein aggregation according to the "prion-like" propagation mechanism [2] that may represent an early event in $\mathrm{AD}$ and proteinopathies.

\section{References}

[1] Kalra H, Drummen GPC, Mathivanan S. Focus on extracellular vesicles: introducing the next small big thing. Int $\mathrm{j} \mathrm{Mol}$ Sci. (2016);17(2):170

[2] Acquatella-Tran Van Ba I, Imberdis T, Perrier V. From prion diseases to prion-like propagation mechanisms of neurodegenerative diseases. Int J Cell Biol. (2013); 2013:975832

\section{Clinical, biological and} neuroradiological evolution of Cerebral Amyloid Angiopathy-related inflammation: the iCA $\beta$ International Network longitudinal study

\author{
M. Longoni ${ }^{1}$, L. Totino ${ }^{1}$, J. DiFrancesco ${ }^{1}$, \\ S. Andreoni ${ }^{1}$, F. Piazza ${ }^{1,2}$ \\ ${ }^{1}$ The inflammatory cerebral amyloid angiopathy \\ and Alzheimer's disease Biomarkers (iCAB) \\ International Network. School of Medicine and \\ Surgery, Milan Center for Neuroscience \\ (NeuroMi), University of Milano-Bicocca (Monza) \\ ${ }^{2}$ The iCAB-ITALY Study Group of the Italian \\ Society, University of Milano-Bicocca (Monza)
}

Objective: Cerebral amyloid angiopathy-related inflammation (CAA-ri) is a rare and aggressive meningoencephalitis characterized by the acute onset of neurological symptoms associated with radiological evidence of cerebral vasogenic edema (VE) and cortical/subcortical microhemorrhages (MH). Here, we described the clinical, neuroradiological and biological evolution of CAA-ri, to provide future directions for the proper management of this challenging disease.

Patients: From the iCA $\beta$ International Network, we identified those patients with a follow-up of $>12$ months. All patients were diagnosed as possible, probable or definitive CAA-ri according to the Auriel 2016 diagnostic criteria.

Methods: VE and MHs were evaluated on fluidattenuated inversion recovery (FLAIR) and T2*weighted gradient echo (T2*-GRE) or susceptibility weighted imaging (SWI) MRI. CSF autoantibodies, TFPI, Abeta and tau were assessed. Clinical and therapeutic findings recorded.

Results: $2 / 60$ patients were identified to undergo CAA-ri recurrence, respectively at two and seven years after first presentation. At recurrence, VE colocalized with the appearance of new MHs, affecting brain areas different from those where the first event occurred. CSF autoantibodies and TFPI revealed as good diagnostic and prognostic biomarkers of VE and MHs, respectively.

Discussion and Conclusions: Our observations improve the understanding of the natural history of CAA-ri, highlighting the importance of a prompt diagnosis, particularly in patients who have already experienced a previous episode of CAA-ri. Although the frequency of late CAA-ri recurrence is rare, in 
the suspect of relapse, a prompt clinical and radiological follow-up should be considered, to obtain a timely diagnosis and medication with strong impact on the patients' outcome.

\section{References}

[1] Piazza F, Greenberg SM, Savoiardo M, Gardinetti M, Chiapparini L, Raicher I, Nitrini R, Sakaguchi H, Brioschi M, Billo G, Colombo A, Lanzani F, Piscosquito G, Carriero MR, Giaccone G, Tagliavini F, Ferrarese C, DiFrancesco JC. Antiamyloid beta autoantibodies in cerebral amyloid angiopathy related inflammation: implications for amyloid-modifying therapies. Annals of Neurology (2013); 73:449-58

[2] DiFrancesco JC, Longoni M, and Piazza F. Anti-A $\beta$ autoantibodies in amyloid related imaging abnormalities (ARIA): candidate biomarker for immunotherapy in Alzheimer's disease and cerebral amyloid angiopathy. Front. Neurol. (2015); 25(6):207

[3] Auriel E, Charidimou A, Gurol ME, Ni J, Van Etten ES, Martinez-Ramirez S, Boulouis G, Piazza F, DiFrancesco JC, Frosch MP, Pontes-Neto OV, Shoamanesh A, Reijmer Y, Vashkevich A, Ayres AM, Schwab KM, Viswanathan A, Greenberg S. Validation of Clinicoradiological Criteria for the Diagnosis of Cerebral Amyloid Angiopathy-Related Inflammation. JAMA Neurol. (2016); 73(2):197-202

\section{Multi-functional liposomes prevent memory impairment and slow down brain B-amyloid deposition in a mouse model of Alzheimer's disease}

\author{
S. Mancini ${ }^{1}$, C. Balducci², F. Re', G. Forloni², \\ M. Masserini ${ }^{1}$ \\ ${ }^{1}$ School of Medicine and Surgery, University of \\ Milano-Bicocca (Monza) \\ ${ }^{2}$ Department of Neuroscience, IRCCS-Mario Negri \\ Institute for Phamarcological Research (Milano)
}

Objectives: In the present study, we evaluated the possibility to delay the progression of Alzheimer's disease (AD) in vivo by means of an early intervention using multi-functional liposomes previously designed for AD therapy and already proved effective in $\mathrm{AD}$ mouse models displaying evident amyloid plaques and cognitive deficit.

Materials: Used liposomes (mApoE-PA-LIP) were functionalized with an Apolipoprotein-E-derived peptide ( $\mathrm{mApoE}$ ) to cross the blood-brain barrier and with phosphatidic acid (PA) to bind $\mathrm{A} \beta$. For the purpose of a disease-modifying treatment, APP/ PS1 mice were used at a presymptomatic age.

Method: mApoE-PA-LIP were administered weekly for 7 months in 5-month-old APP/PS1 mice, periodically tested for memory performance. At the end of the treatment, half of the animals was sacri- ficed and organs collected and analyzed to assess the effects on $A \beta$ pathology. The rest of the animals was sacrificed three months after the end of the treatment and analyzed for the same parameters to evaluate the duration of treatment effects.

Results: mApoE-PA-LIP treatment prevented the loss of long-term recognition memory and hindered the deposition of $A \beta$ plaques $(-30 \%)$ in the brain of treated mice. In parallel, $A \beta$ levels were found increased in the liver $(+30 \%)$. The same effects were visible three months after the end of the treatment.

Discussion: These findings suggest that mApoEPA-LIP treatment promotes removal of $A \beta$ from the brain and its peripheral clearance, effects that persist even after treatment completion.

Conclusions: Together, these findings promote multi-functional liposomes as a valuable approach potentially suitable for delaying relevant features of $\mathrm{AD}$.

\section{References}

[1] Bana L, Minniti S, Salvati E, Sesana S, Zambelli V, Cagnotto A, Orlando A, Cazzaniga E, Zwart R, Scheper W, Masserini $\mathrm{M}, \mathrm{Re}$ F. Liposomes bi-functionalized with phosphatidic acid and an ApoE-derived peptide affect $\mathrm{A} \beta$ aggregation features and cross the blood-brain-barrier: implications for therapy of Alzheimer disease. Nanomedicine (2014);10(7):1583-90

[2] Balducci C, Mancini S, Minniti S, La Vitola P, Zotti M, Sancini G, Mauri M, Cagnotto A, Colombo L, Fiordaliso F, Grigoli E, Salmona M, Snellman A, Haaparanta-Solin M, Forloni G, Masserini M, Re F. Multifunctional liposomes reduce brain $\beta$-amyloid burden and ameliorate memory impairment in Alzheimer's disease mouse models. J Neurosci (2014);34(42):14022-31

\section{Effects of oxidative stress and inflammation induced by ultrafine particles on Alzheimer disease onset}

\author{
C. Milani, F. Farina, L. Botto, E. Lonati,
} G. Sancini, A. Bulbarelli, P. Palestini

Department of Medicine and Surgery, Milan Center of Neuroscience, University of Milan Bicocca (Monza)

Objectives: The aim of this work was to evaluate the activation of oxidative stress and inflammation in mice exposed to ultrafine particles (UFPs), and to elucidate putative physiopathological correlations with neurodegeneration.

Materials: UFPs derived from two different anthropogenic sources were used: DEP, from EURO 4 diesel engine emission, and $\mathrm{BC}$, from pellet boiler biomass emission (ENEA). 
Method: Male BALB/c mice were submitted to single or repeated intratracheal instillation of DEP and $\mathrm{BC}(50 \mu \mathrm{g})$; in parallel, control mice were always considered (sham). Cortex, cerebellum and hippocampus from sham and treated mice were screened for markers of inflammation and oxidative stress (iNOS, HO-1), endoplasmic reticulum stress (Hsp70), a putative pro-carcinogenic marker (Cyp1B1) and markers related to the onset of Alzheimer Disease (AD) (P-Tau, Tau, P-APP, APP, BACE1).

Results: After both single and repeated DEP and $\mathrm{BC}$ instillation, we observed an increase in iNOS, HO-1 and Hsp70 levels in all districts, while Cyp1B1 and markers of neurodegeneration changed only after repeated treatment.

Discussion: The single DEP and BC instillation induced oxidative stress, endoplasmic reticulum stress and inflammation, while typical AD-related proteins seemed not affected. The repeated instillation was able to sustain oxidative stress and inflammation; moreover, it induced increase of BACE1 levels and changes in Tau and APP total levels and phosphorylation. Finally, BC seemed to be less effective than DEP.

Conclusions: Our results suggest UFPs or inflammatory mediators translocation from lungs, and their effects on the brain, after both acute and repeated DEP and BC treatment.

Grant by Fondazione Cariplo.

\section{References}

[1] Genc S., Zadeoglulari Z., Fuss S.H., Genc K. The adverse effects of air pollution on the nervous system. J Toxicol. (2012);2012:782462

[2] Levesque S., Surace M.J., McDonald J., Block M.L. Air pollution \& the brain: Subchronic diesel exhaust exposure causes neuroinflammation and elevates early markers of neurodegenerative disease. J Neuroinflammation (2011);8:105

[3] Block M.L. and Calderón-Garcidueñas L. Air pollution: mechanisms of neuroinflammation and CNS disease. Trends Neurosci. (2009);9:506-516

\section{Effect of modified collagen on amyloid beta peptide aggregation}

F. Nicotra ${ }^{1}$, L. Russo ${ }^{1}$, F. Re ${ }^{2}$, M. Masserini ${ }^{2}$, A. Sassella ${ }^{3}$, S. Trabattoni ${ }^{3}$, L. Cipolla ${ }^{1}$

${ }^{1}$ Department of Biotechnology and Biosciences, University of Milano-Bicocca (Milano)

${ }^{2}$ Department of Medicine and Surgery, University of Milano-Bicocca (Milano)

${ }^{3}$ Department of Materials Science, University of Milano-Bicocca (Milano)
Objectives: Aim of the work is the synthesis and functionalization of collagen based nanostructured materials in order to evaluate their influence on neurodegenerative diseases, affecting the A $\beta 42$ aggregation or disaggregation.

Materials and methods: Collagen based materials were produced by a solvent-casting method as previously described [ ], and functionalized using $\gamma$-thiobutyrolactone in order to obtain the thiolated collagen (Collagen-SH) [2]. A $\beta 42$ monomeric or fibrillary preparations were obtained from the commercial peptide (Sigma-Aldrich). The effect of collagen matrices on the $A \beta 42$ aggregation (from monomers-enriched preparations) or disaggregation (from fibrils-enriched preparations) were evaluated by the ThT assay.

Discussion and Results: The results showed that monomers-enriched preparations of Ab42 alone displayed an increase in the fluorescence intensity over the time, indicating the aggregation of the peptide. The incubation of monomers-enriched Ab42 with collagen matrices up to 5 days showed a lower increase in FI respect to the $\mathrm{Ab} 42$ alone over the time.

Conclusions: These preliminary results suggest that the functionalized collagen matrices could tackle the process of Abeta aggregation.

\section{References}

[1] L Russo, A Sgambato, M Lecchi, V Pastori, M Raspanti, A Natalello, SM. Doglia, F Nicotra, L Cipolla Neoglucosylated collagen matrices drive neuronal cells to differentiate. ACS Chem Neurosci. (2014);5:261-5

[2] Laura Russo, Chiara Battocchio, Valeria Secchi, Elena Magnano, Silvia Nappini, Francesca Taraballi, Luca Gabrielli, Francesca Comelli, Antonio Papagni, Barbara Costa, Giovanni Polzonetti, Francesco Nicotra, Antonino Natalello, Silvia M. Doglia, and Laura Cipolla. Thiol-ene Mediated Neoglycosylation of Collagen Patches: A Preliminary Study. Langmuir. (2014);30:1336-1342

\section{Alzheimer's Disease and Mild Cognitive Impairment: extracellular and intracellular redox alterations}

\author{
W. Rungratanawanich ${ }^{1}$, M. Marziano ${ }^{2}$, G. Abate ${ }^{2}$, \\ D. Uberti \\ ${ }^{1}$ Horwang School, University of Brescia (Brescia) \\ ${ }^{2} D M M T$, University of Brescia (Brescia)
}

Objectives: This study focused on the alterations of extracellular and intracellular redox potential in plasma and peripheral blood cell (PBMC) of Alzheimer's Disease (AD), Mild Cognitive 
Impairment (MCI) patients and cognitively healthy subjects (CHS).

Materials: A total of 120 participants from Spain were enrolled including 42 individuals of $\mathrm{AD}, 36$ of $\mathrm{MCI}$ and 42 of CHS.

Method: All subjects were examined and classified by behavioral neurologists with currently reviewed criteria. Antioxidant enzyme activities, Superoxide Dismutase (SOD), Catalases (CAT) and Glutathione Peroxidases (GPx), were measured by enzymatic assays.

Results: SOD activity was significantly reduced $(\mathrm{p}<0.05)$ in both plasma and PBMC of AD and MCI groups comparing with CHS. On the other hand, GPx activity was increased particularly in plasma of MCI patients.

Discussion: The three antioxidant enzymes work in concert to prevent an excessive oxidative stress. The results found that only SOD activity was significantly decreased. However, unexpected enhancement GPx was investigated, suggesting provisional compensatory mechanisms to take pro-oxidants under control.

Conclusions: Antioxidant enzyme activity measurements illustrated the evolvement of extracellular and intracellular unbalance redox potential in $\mathrm{AD}$ pathology. It is possible that redox profile in plasma and PBMC may benefit to the early AD diagnosis.

\section{References}

[1] Guzmán-Martínez L, Farías GA, Maccioni RB. Emerging noninvasive biomarkers for early detection of Alzheimer's disease. Arch Med Res. (2012) Nov;43(8):663-6. doi:10.1016/j.arcmed.2012.10.013.

[2] Khan TK, Alkon DL. Peripheral biomarkers of Alzheimer's disease. J Alzheimers Dis. (2015) Jan 1;44(3):729-44. doi: 10.3233/JAD-142262.

[3] Sohal RS, Mockett RJ, Orr WC. Mechanisms of aging: an appraisal of the oxidative stress hypothesis. Free Radic Biol Med. (2002) Sep 1;33(5):575-86

\section{Factors contributing to therapeutic potential of oral galactose in a rat model of sporadic Alzheimer's disease}

\author{
M. Salkovic-Petrisic ${ }^{1}$, A. Knezovic ${ }^{1}$, J. Osmanovic \\ Barilar $^{1}$, W. Reutter ${ }^{2}$ \\ ${ }^{1}$ Department of Pharmacology/Croatian Institute \\ for Brain Research, University of Zagreb, School of \\ Medicine (Zagreb-HR) \\ ${ }^{2}$ Institute of Laboratory Medicine, Clinical \\ Chemistry and Pathobiochemistry, Charité \\ University (Berlin-Dahlem-D)
}

Objectives: Galactose demonstrates insulin-independent entry into the cell and intracellular metabolism to glucose suggesting possible therapeutic potential for brain insulin resistance and glucose hypometabolism in sporadic Alzheimer's disease (sAD). Our previously reported that chronic oral galactose administration prevents/improves cognitive deficit in streptozotocin-intracerebroventricularly (STZ-icv) treated rat model of $\mathrm{sAD}(1)$. We aimed to explore galactose levels in blood/cerebrospinal fluid (CSF) of healthy and STZ-icv rats following different galactose doses and routes of administration.

Materials: Healthy Wistar rats were given galactose intraperitoneally (10 mg; $200 \mathrm{mg}$, ip) or orally (200 mg;1000 mg, po). Another group was treated STZ-icv (3 mg/kg), and subjected to ip/po $200 \mathrm{mg}$ galactose dose after 1 month and euthanized $15 \mathrm{~min}$ afterwards.

Methods: Blood/CSF galactose was measured by ELISA, cognition by Passive avoidance test, and data analysed by Kruskal-Wallis ANOVA/MannWhitney U- test, $\mathrm{p}<0.05$.

Results: Doses of $\geq 200 \mathrm{mg}$ resulted in blood galactose increment regardless route of administration $(p<0.05)$ in healthy and STZ-icv rats. Increment in CSF was seen only in healthy rats after $1000 \mathrm{mg}$ po $(136 \%)$ and $200 \mathrm{mg}$ ip (114\%) dose (p>0.05). 200 $\mathrm{mg}$ dose induced larger blood galactose increment following ip than po administration $(220 \%$ vs $121 \%$, $\mathrm{p}<0.05$ ), with CSF galactose increment detected only after ip administration, both seen in healthy rats. STZ-icv-induced cognitive deficits remained unaffected by single galactose dose.

Discussion: Oral vs parenteral galactose elicits significantly milder rise in its blood/CSF levels.

Conclusion: Lower blood/CSF galactose levels might account for cognitive improvement seen after chronic oral vs parenteral galactose treatment.

Supported by HRZZ-IP-2014-09-4639.

\section{Reference}

[1] Salkovic-Petrisic M, Osmanovic-Barilar J, Knezovic A, Hoyer S, Mosetter K, Reutter W. Long-term oral galactose treatment prevents cognitive deficits in male Wistar rats treated intracerebroventricularly with streptozotocin. Neuropharmacology (2014);77:68-80 


\section{New method for early detection of CNS damage in elderly subjects: abnormal lipid accumulation and cluster formation in naìve PBMCs}

\author{
L. Serchisu ${ }^{1}$, D. Costaggiu ${ }^{2}$, C. Abete ${ }^{1}$, A. Mandas ${ }^{1}$ \\ ${ }^{1}$ Department of Medical Sciences, University of \\ Cagliari (Cagliari) \\ ${ }^{2}$ School of Specialisation in Geriatrics, University \\ of Cagliari (Cagliari)
}

Objectives: To ascertain whether neutral lipid accumulation (NLs) and cellular clusters formation (CC) in Peripheral Blood Mononuclear Cells taken from fresh blood (naive PBMCs) represented early indicators of neurodegeneration in $\geq 65$ years old subjects.

Material: $192 \geq 65$ years old subjects enrolled at our geriatric unit. Twenty patients showed Alzheimer's disease (AD), 14 mixed dementia (MD) and 34 vascular dementia (VD); 29 showed mild cognitive impairment (MCI); 95 cognitively normal patients were used as controls (C).

Methods: NLs of PBMCs were stained by oil red $\mathrm{O}$ (ORO) method and imaged using an inverted phase microscope fitted with a digital camera. Red intensity and CC were scored on semiquantitative scales.

Results: Subjects with any type of dementia (D) had higher ORO scores in PBMCs than controls (C). ORO score did not significantly differ between $\mathrm{AD}$, $\mathrm{MD}$ and VD. In MCI, it was significantly higher than $C$, significantly lower than $A D, M D$ and VD. Significant inverse correlation was found between ORO and MMSE scores $(\mathrm{r}=-0.641, \mathrm{P}<0.001)$. $\mathrm{CC}$ showed a similar pattern to ORO, being significantly lower in $\mathrm{C}$ than $\mathrm{D}$, and higher in D than MCI. CC score negatively correlated with MMSE $(\mathrm{r}=-0.573$, $\mathrm{P}<0.001)$, positively with ORO $(\mathrm{r}=0.87, \mathrm{P}<0.001)$.

Discussion: This study suggests that NL accumulation in PBMCs from all dementia types patients and shows a similar pattern of CC.

Conclusions: Our findings suggest naive PBMC cytoplasm NL accumulation and CC tendency represent a simple, economical and non-invasive approach to determine and monitor neuronal damage in the early phases of neurodegenerative illnesses.

\section{References}

[1] Mandas A, Dessì S. Mononuclear cells in dementia. Clin Chim Acta (2014);431C:278-287. doi: 10.1016/j.cca.2014.02.016
[2] Pani A, Mandas A, Diaz G, Abete C, Cocco PL, Angius F, et al. Accumulation of neutral lipids in peripheral blood mononuclear cells as a distinctive trait of Alzheimer patients and asymptomatic subjects at risk of disease. BMC Med (2009);7:66. doi: 10.1186/1741-7015-7-66

[3] Pani A, Dessì S, Diaz G, La Colla P, Abete C, Mulas C, et al. Altered cholesterol ester cycle in skin fibroblasts from patients with Alzheimer's disease. J Alzheimers Dis (2009);18(4):829-841.doi:10.3233/JAD-2009-1193

\section{The toxicity of amyloid-beta oligomers and tau hyperphosphorylation are prevented from lipid-based nanoparticles in human primary fibroblasts}

\author{
C. P. Zoia, E. Conti, D. Cereda, V. Rodriguez- \\ Menendez, S. Andreoni, M. Gregori, S. Sesana, \\ M. Masserini, L. Tremolizzo, C. Ferrarese \\ School of Medicine, Milan Center for \\ Neuroscience, Milano-Bicocca (Monza)
}

Objective: Abeta leads to neurodegeneration and memory impairment via critical signal transduction processes, both at central and peripheral level. Studying the oligomeric-Abeta toxicity could helpful to clarify these phenomena, and to develop therapeutic and effective prevention technologies [1-3]. Nanoparticles (NPs) biocompatibility and their ability to modulate Abeta toxicity were investigated in primary cultures from human fibroblasts.

Methods: Control fibroblasts were incubated with Abeta oligomers and NP, functionalized or not with a ApoE monomer (mApo). By MTT assay we evaluate energetic metabolism and cell viability. Western Blot and p-Elisa were performed to evidence any modulation of MAPK and Tau hyperphosphorylation. By uptake studies and confocal images, we investigated the involvement of NPs in modulating the Abeta-induced excitotoxicity and their interaction with oligomers. By commercial kit, malondialdehyde levels were tested.

Results: NPs do not affect cell viability, they prevent the Abeta-induced deficit of mitochondrial activity $(\mathrm{p}<0.001)$ and MAPK phosphorylation $(\mathrm{p}<0.001)$. mApo cross cell membrane, bind oligomers and modulate glutamate uptake in a dose dependent manner $(p<0.01)$; they counteract the increase of Abeta-induced lipid peroxidation $(\mathrm{p}<0.002)$ and Tauhyperphosphorylation $(\mathrm{p}<0.0001)$.

Conclusion: These results suggest that NPs could represent a useful tool to antagonize Abeta oligomers toxicity, counteracting the cellular stress, pro- 
tein epigenetic regulation and glutamate cytotoxicity. The modulations of MAPK pathways, altered by Abeta oligomers, might clarify any molecular mechanisms that mirror those involved in the early AD phases, and NPs may be useful to study new potential therapeutic strategies.

\section{References}

[1] Kumar A, Singh A, Ekavali. A review on Alzheimer's disease pathophysiology and its management: an update. Pharmacol Rep (2015);67(2):195203

[2] Qi H, Prabakaran S, Cantrelle FC, Chambraud BE, et al. Characterization of Neuronal Tau Protein as a Target of Extracellular-signal-regulated Kinase. J Biol Chem (2016);pii: jbc.M115.700914.

[3] Gregori M, Masserini M, Mancini S. Nanomedicine for the treatment of Alzheimer's disease. Nanomedicine (Lond). (2015);10(7):1203-18

\section{NEUROIMAGING}

\section{Molecular Imaging of Microglia activation in response to acute neurodegenerative damage in TREM2 deficient mice}
S. Belloli ${ }^{1}$, M. Pannese ${ }^{2}$, I. Raccagni ${ }^{3}$, G. Di Grigoli ${ }^{1}$, V. Murtaj ${ }^{3}$, A. Carpinellii ${ }^{1}$, C. Monterisi ${ }^{3}$, P. Panina-Bordignon ${ }^{2}$, R. Moresco ${ }^{3}$ ${ }^{1}$ Institute of Molecular Bioimaging and Physiology, CNR (Segrate)
${ }^{2}$ Division of Research in Genetics and Cell Biology, San Raffaele Scientific Institute (Milano)
${ }^{3}$ Department of Medicine and Surgery, University of Milan-Bicocca (Monza)

Aim: Mutations in the TREM2 gene confer risk for $\mathrm{AD}$ and susceptibility for PD. We evaluated the effect of TREM2 KO in MPTP model, measuring neurodegeneration and microglia activation using [11C] FECIT and [11C]PK11195.

Material and Methods: [11C]FECIT biodistribution was measured in wt animals after MPTP at 1,2 and 7 days or PBS. TREM2-/- and wt animals were evaluated at 7 days by [11C]FECIT-PET. [11C] PK11195 biodistribution was performed in wt animals after MPTP at 1, 2 and 7 days or PBS. Thereafter, [11C]PK11195 biodistribution was evaluated 1 day in wt and TREM2-/-. Neuroinflammation markers were measured in additional animals.

Results: In wt mice, MPTP induced progressive [11C]FECIT uptake decrease, culminating 7 days after. In striatum, SN and cerebellum TREM2 expression significantly increased between 2 and 7 days. TNF $\alpha$ and Galectin-3 mRNA were maximum in striatum at day 1 as Iba-1 immunofluorescence; while IL-1 $\beta$, IL-4 and TSPO were higher in SN at 7 days. [11C]PK11195 uptake and GFAP immunostaining was highest in the striatum at day 2 postMPTP. In TREM2-/- mice, MPTP induced a decline of [11C]FECIT uptake similar to wt animals but [11C]PK11195 was maximum at 1 day accompanied by reduction in TNF $\alpha$ and IL-1 $\beta$ in striatum. IL-4 in TREM2-/- was significantly higher than in wt animals while Iba-1 was higher in wt than in TREM2-/PBS animals.

Discussion and Conclusion: TREM2 expression correlates with a specific activated microglia phenotype with a possible protective function. Inflammation markers expression, suggest a potential role of TREM2 in the switch-off of proinflammatory processes.

\section{Correlations between neuropsychological tests and structural brain magnetic resonance imaging in a cohort of patients with mild cognitive impairment}
L. Biscetti ${ }^{1}$, N. Salvadori ${ }^{1}$, P. Eusebi ${ }^{1}$, E. Chipi ${ }^{1}$,
L. Farotti ${ }^{1}$, M. Russo ${ }^{1}$, R. Tarducci ${ }^{2}$, P. Chiarini ${ }^{3}$, P. Floridi ${ }^{3}$, P. Calabresi ${ }^{1}$, L. Parnetti ${ }^{1}$
${ }^{1}$ Memory Clinic, Neurology, University of Perugia (Perugia)
${ }^{2}$ Medical Physics Unit, Perugia General Hospital
(Perugia)
${ }^{3}$ Neuroradiology, University of Perugia (Perugia)

Objectives: To assess the correlations between neuropsychological tests and magnetic resonance imaging (MRI) brain volumes in a cohort of well characterized patients with mild cognitive impairment (MCI).

Patients: Twenty-five consecutive MCI patients referred to our Memory Clinic.

Methods: All the patients underwent serial MRI 3T, standard neuropsychological examination and tests from the Cambridge Neuropsychological Test Automated Battery (CANTAB) [1] every 6 months in a 2-year follow-up period. Brain volumes were measured using the FreeSurfer software [2]. In this study, we have calculated correlations between neuropsychological and MRI measures at baseline with Spearman coefficients. 
Results: At baseline, better scores on Spatial Recognition Memory (SRM) and Delayed Matching-tosample (DMS) test from CANTAB correlated with left putamen volume $(\mathrm{r}=0.52, \mathrm{p}<0.01$ and $\mathrm{r}=0.54$, $\mathrm{p}<0.01$ respectively). ADAS-Cog scores [3] inversely correlated with left $(\mathrm{r}=-0.56, \mathrm{p}<0.01)$ and right hippocampus $(\mathrm{r}=-0.62, \mathrm{p}<0.001)$ and left amygdala $(\mathrm{r}=-0.52, \mathrm{p}<0.01)$ volumes.

Discussion: SRM and DMS scores, respectively exploring executive functions and delayed recognition memory, correlated with left putamen volumes, supporting the idea that performances on these CANTAB tasks involve fronto-subcortical networks. The inverse correlation between ADAS-Cog total score and hippocampal volume confirms the accuracy of this test in assessing memory disturbances.

Conclusion: We found consistent correlations between neuropsychological test and MRI measures at baseline. Longitudinal observation of these parameters will add information about the parallelism of clinical and structural changes taking place in these subjects.

\section{References}

[1] Robbins TW, James M, Owen AM, Sahakian BJ, McInnes L, Rabbitt P. Cambridge Neuropsychological Test Automated Battery (CANTAB): a factor analytic study of a large sample of normal elderly volunteers. Dementia (1994) SepOct;5(5):266-81.

[2] Fischl B. FreeSurfer Neuroimage (2012) Aug 15;62(2):77481

[3] Alzheimer's Disease Assessment Scale - Cognitive (ADASCOG) (Adapted from the Administration and scoring Manual for the Alzheimer's disease Assessment scale, 1994 Revised Edition, Richard C. Mohs, PhD. Copyright (C) 1994 by the Mount Sinai School of Medicine. Present Manual modified by: Donald Connor, PhD. Kimberly Schafer, MS (3/98)

\section{Monitoring Neuroinflammation with the TSPO tracer [18F]VC701, after LPS systemic administration in adult and aged mice}

\author{
G. Di Grigoli ${ }^{1}$, S. Belloli ${ }^{1}$, V. Murtaj ${ }^{2}$, \\ C. Monterisi ${ }^{2}$, V. Masiello ${ }^{3}$, R. Moresco ${ }^{1}$ \\ ${ }^{1}$ Institute of Molecular Bioimaging and Physiology, \\ CNR, Milan Center for Neuroscience, UNIMIB \\ (Segrate-MI) \\ ${ }^{2}$ Department of Medicine and Surgery, University \\ of Milan-Bicocca (Milano) \\ ${ }^{3}$ Nuclear Medicine Department, San Raffaele \\ Scientific Institute (Milano)
}

Aim: Aim of the study is monitoring brain inflammation and its modulation by gender and age after systemic administration of LPS. To this end, we studied the biodistribution of the high-affinity tracer specific for TSPO, [18F]VC701, in mice treated with the neurotoxin.

Material and Methods: [18F]VC701 biodistribution was measured in WT animals 6 hours and 3 months after systemic injection of LPS/vehicle in young and aged C57BL/6 mice. To investigate the gender modulation of microglia activation, studies were performed in both male and female mice. $2 \mathrm{~h}$ after tracer injection, animals were sacrificed and brain regions processed for gamma counting.

Results: LPS treatment caused significant increase of [18F]VC701 uptake in aged males compared to vehicle treated animals, 6 hours after toxin administration (ctx SUV, LPS aged vs Vehicle aged: $0.47 \pm$ 0.06 vs $0.26 \pm 0.07, \mathrm{p}<0.05$ ). Moreover, aged males showed uptake values at 6 hours higher than those observed for young animals (ctx SUV, LPS aged vs LPS young: $0.47 \pm 0.06$ vs $0.24 \pm 0.05, p<0.05$ ) suggesting a possible additional age effect in activating microglia. As confirmation of age effect on microglia activation, both male and female control animals, showed increased trend brain uptake with aging.

Discussion and Conclusion: Data obtained from this work confirmed the feasibility of [18F]VC701 imaging to monitor Neuroinflammation. [18F] VC701-LPS paradigm could be of interest to test new anti-inflammatory drugs for neurodegenerative disease.

\section{Cognitive and functional metabolic profiles in primary progressive aphasia variants}

L. C. Greco ${ }^{1}$, C. Cerami ${ }^{2}$, A. Dodich ${ }^{2}$, L. Iaccarino ${ }^{2}$, S. Iannaccone ${ }^{3}$, G. Magnani ${ }^{4}$, D. Perani ${ }^{2}$

${ }^{1}$ Centro Clinico NEMO-Fondazione Serena Onlus, Vita Salute-San Raffaele (Milano)

${ }^{2}$ San Raffaele Scientific Institute, Vita-Salute San

Raffaele University (Milano)

${ }^{3}$ San Raffaele Scientific Institute, San Raffaele

Turro Hospital (Milano)

${ }^{4}$ San Raffaele Scientific Institute, San Raffaele Hospital (Milano)

Background and Objective: Primary Progressive Aphasia (PPA) represents a heterogeneous group of neurodegenerative conditions in which language 
impairment is the main symptom. Three main variants exist: the semantic (sv-PPA), the non fluent/ agrammatic (nfv-PPA), and the logopenic/phonologic (lv-PPA). In this study, we aimed at investigating the different cognitive and FDG-PET metabolic profiles to provide a comprehensive view on the cognitive-functional correlates in the three variants, paying attention also to case-to-case variability.

Material and Methods: 48 PPA patients (i.e., 11 sv-PPA, 19 nfv-PPA and 18 lv-PPA) were included. The nfv-PPA group included pure nfv-PPA cases $(n=8)$ as well as combined cases with atypical parkinsonism ( $\mathrm{n}=11)$. Neuropsychological information as well as FDG-PET scans were acquired at close time. FDG-PET data were analysed using an optimized voxel-based SPM method at the single-subject level. Second-level group analysis was performed to evaluate commonalities in brain metabolic patterns.

Results: Different language profiles characterized the PPA variants, while nfv-PPA and lv-PPA groups presented deficits in non-language domains. Different patterns of brain hypometabolism characterized the single-subject level analysis, while whole brain group analyses on PPA variants confirmed the patterns of brain hypometabolism characterizing each variant in agreement with literature reports.

Discussion and Conclusion: The study highlighted the distinctive cognitive and functional features of the three PPA variants at the single subject level, adding novel evidence to current literature mainly limited to MRI studies in group analysis distinguishing the PPA variants. Our data provide first evidence for the utility of the FDG-PET SPM single-subject analysis to support early differential diagnosis of PPAs.

\section{References}

[1] Gorno-Tempini ML, Hillis AE, Weintraub S, Kertesz A, Mendez M, Cappa SF, Ogar JM, Rohrer JD, Black S, Boeve BF, Manes F, Dronkers NF, Vandenberghe R, Rascovsky K, Patterson K, Miller BL, Knopman DS, Hodges JR, Mesulam MM, Grossman M. Classification of primary progressive aphasia and its variants. Neurology (2011) Mar 15;76(11):1006-14. doi: 10.1212/WNL.0b013e31821103e6. Epub 2011 Feb 16.

[2] Della Rosa PA, Cerami C, Gallivanone F, Prestia A, Caroli A, Castiglioni I, Gilardi MC, Frisoni G, Friston K, Ashburner J, Perani D. EADC-PET Consortium. A standardized [18F]FDG-PET template for spatial normalization in statistical parametric mapping of dementia. Neuroinformatics (2014) Oct;12(4):575-93. doi: 10.1007/s12021-014-9235-4.

[3] Perani D, Della Rosa PA, Cerami C, Gallivanone F, Fallanca F, Vanoli EG, Panzacchi A, Nobili F, Pappatà S, Marcone A, Garibotto V, Castiglioni I, Magnani G, Cappa SF, Gianolli L. EADC-PET Consortium. Validation of an optimized SPM procedure for FDG-PET in dementia diagnosis in a clinical setting. Neuroimage Clin. (2014) Oct 10.1016/j. nicl.2014.10.009. eCollection 2014. 24;6:445-54

\section{Toward the identification of functional biomarkers for Alzheimer's disease: a magnetoencephalography study}

F. Jacini ${ }^{1}$, P. Sorrentino ${ }^{2}$, R. Rucco ${ }^{1}$, P. Varriale ${ }^{3}$, V. Agosti ${ }^{4}$, F. Baselice ${ }^{5}$, A. Sorriso ${ }^{5}$, G. Sorrentino ${ }^{6}$

${ }^{1}$ Department of Motor Sciences and Wellness, University of Naples "Parthenope" (Napoli)

${ }^{2}$ Department of Clinical Neurophysiology and MEG Center, VU University Medical Center

(Amsterdam-NL)

${ }^{3}$ Institut du Cerveau et de la Moelle épinière, ICM (Paris-F)

${ }^{4}$ Institute for Diagnosis and Cure "Hermitage

Capodimonte" (Napoli)

${ }^{5}$ Department of Engineering, University of Naples

"Parthenope" (Napoli)

${ }^{6}$ Institute of Applied Sciences and Intelligent

Systems “E. Caianiello” CNR (Pozzuoli-NA)

Aim of the work is to identify a functional biomarker able to detect connectivity changes in Mild Cognitive Impairment (MCI) patients. A reliable biomarker for early Alzheimer's disease (AD) could improve clinical management.

Materials: We recruited 11 MCI patients and 13 control subjects. Participants underwent a 5 minutes resting-state magnetoencephalography (MEG) record.

Methods: The signals were cleaned from environmental and physiological artefacts. Ten clean epochs were bandpass filtered in the canonical frequency bands. To assess functional connectivity we used the phase lag time. An adjacency matrix, for each epoch in each frequency band, was obtained. The Minimum Spanning Tree (MST) was calculated for each adjacency matrix. Several network metrics were computed, and compared by permutation testing corrected for multiple comparisons.

Results: In the delta band, the tree hierarchy (Th) and the leaf fraction (L) were found to be higher in the MCI group as compared to controls. Th is a measure that captures the fine tuning of the network between a star-like and a line-like network conformation. L represents the fraction of nodes that have degree (number of links) equal to 1 .

Discussion: These findings indicate a greater tendency towards segregation in MCI that can be interpreted as a compensatory mechanism occurring 
before there is overt clinical involvement. These changes seem to be driven by a global feature of the network rather than of a specific area.

Conclusions: Network theory applied to MEG data could lead to the identification of biomarkers for early $\mathrm{AD}$.

\section{Reference}

[1] Stam CJ, Tewarie P, Van Dellen E, van Straaten ECW, Hillebrand A, Van Mieghem P. The trees and the forest: Characterization of complex brain networks with minimum spanning trees. Int J Psychophysiol (2014);92:129-138

\section{Gender Differences in Aging and Alzheimer's Dementia: an 18F-FDG- PET Study}

\author{
M. Malpetti ${ }^{1}$, T. Ballarini², L. Presotto ${ }^{2}$, \\ V. Garibotto ${ }^{3}$, D. Perani ${ }^{1}$ \\ ${ }^{1}$ San Raffaele Scientific Institute, Vita-Salute San \\ Raffaele University (Milano) \\ ${ }^{2}$ Division of Neuroscience, San Raffaele Scientific \\ Institute (Milano) \\ ${ }^{3}$ Division of Nuclear Medicine and Molecular \\ Imaging, Geneva University Hospital (Geneva-CH)
}

Objectives: We investigated gender differences in the effects of cognitive reserve (CR)[1] proxies (i.e. education and occupation) on brain plasticity and brain-cognitive reserve (BCR), as measured by $18 \mathrm{~F}$ FDG-PET metabolic activity.

Materials: 225 healthy-elderly (HE) and $282 \mathrm{Al}-$ zheimer's dementia (AD) patients were selected.

Methods: We tested the effect of gender in voxelwise correlations between 1) aging and brain metabolism in HE; 2) CR-proxies and brain metabolism in both $\mathrm{HE}$ and $\mathrm{AD}$ patients. We also assessed gender differences in seed-based brain-metabolic connectivity (MC).

Results: In HE, males showed more age-related vulnerability than females in frontal medial regions for cognitive control and a strong MC in posterior Default-Mode Network. Males had higher education and occupation attainments as compared to females. These CR-proxies correlated with increased metabolism in posterior-associative cortex in males and in the limbic-affective and the anterior-executive networks in females, consistent also with the MC-analyses. In $\mathrm{AD}$, there was a major $\mathrm{BCR}$ in prefrontal circuit for cognitive control and in socio-affective networks in females, while CR-proxies correlated to hypometabolism in temporo-parietal structures in males. Furthermore, females showed more extended $\mathrm{MC}$ in fronto-parietal and language networks.

Discussion: Our results support the role of CR on BCR (brain metabolism) [2,3], in both aging and $\mathrm{AD}$, with gender differences. These highlight a neuroprotective effect in males for posterior-associative regions, whereas in females for networks involved in cognitive-control and socio-affective processing.

Conclusions: Gender differences in brain plasticity and BCR may be explained by underlying biological determinants and sociodemographic factors (e.g. type and level of education and occupation).

\section{References}

[1] Stern Y. Cognitive reserve in ageing and Alzheimer's disease. The Lancet Neurology (2012);11(11):1006-1012

[2] Arenaza-Urquijo E.M., Landeau B., La Joie R., Mevel K., Mézenge F., Perrotin A., Desgranges B., Bartrés-Faz D., Eustache F. and Chételat G. Relationships between years of education and gray matter volume, metabolism and functional connectivity in healthy elders. Neuroimage (2013);83:450457

[3] Garibotto V., Borroni B., Kalbe E., Herholz K., Salmon E., Holtoff V., Sorbi S., Cappa S.F., Padovani A., Fazio F. and Perani D. Education and occupation as proxies for reserve in aMCI converters and AD FDG-PET evidence. Neurology (2008);71(17):1342-1349

\section{Bilingualism and Brain Reserve in Alzheimer's Dementia}

D. Perani ${ }^{1}$, T. Ballarini' ${ }^{2}$, F. Lubian ${ }^{3}$, M. Malpetti ${ }^{1}$, G. Magnani ${ }^{4}$, M. Farsad ${ }^{3}$, J. Abutalebi ${ }^{1}$

${ }^{1}$ San Raffaele Scientific Institute, Vita-Salute San

Raffaele (Milano)

${ }^{2}$ Division of Neuroscience, San Raffaele Scientific Institute (Milano)

${ }^{3}$ Nuclear Medicine Department, Azienda Sanitaria dell' Alto Adige (Bolzano)

${ }^{4}$ Department of Neurology, San Raffaele Hospital (Milano)

Objectives: Epidemiological evidence suggests that bilingualism may act as cognitive reserve (CR) $[1,2]$, delaying the onset of clinical dementia symptoms to 4-5 years. The aim of this study was to investigate whether bilingualism affects brain neural/ cognitive reserve (BCR) in Alzheimer's disease(AD) patients using FDG-PET for brain metabolism measures and metabolic-connectivity(MC) analyses.

Materials: 85 AD patients were selected: 40monolinguals and $45 \mathrm{German}-\mathrm{Italian}$ bilinguals. For the latter Bilingualism Index (BI) of language daily/ lifelong-usage was assessed. FDG-PET Statistical 
Parametric Maps were obtained in each individual and in a comparison between the AD groups. Correlations between brain metabolism and $\mathrm{BI}$ in the bilingual group, and seed-based inter-regional MC analyses in both groups were performed.

Results: Bilinguals were more than 5 years older than monolinguals. Both groups showed the ADtypical cerebral hypometabolic pattern and, for bilinguals only, there was a more extended hypometabolism in the left hemisphere. Further, BI correlated with brain hypometabolism in the parietal, temporal and sensorimotor cortices, and also with increased metabolism in orbitofrontal, cingulate cortex and inferior frontal gyrus perhaps as a compensation for the greater brain hypometabolism observed in bilingual AD individuals. Finally, MCanalysis showed increased connectivity in frontoparietal executive-control networks and defaultmode network, including cingulate cortex and subcortical structures, only in bilinguals.

Discussion: The results strongly support a greater BCR, compensatory mechanisms and increased strength in the cognitive control systems in bilingual $\mathrm{AD}$ patients, possibly induced by $\mathrm{CR}$.

Conclusions: Our study using 18F-FDG-PET demonstrates that lifelong bilingualism can exert a neuroprotective effect coping with neurodegeneration, acting through neural reserve and compensatory mechanisms.

\section{References}

[1] Perani, D. and Abutalebi, J. Bilingualism, dementia, cognitive and neural reserve. Current opinion in neurology (2015);28(6):618-625

[2] Bialystok, E., Craik, F.I. and Luk, G. Bilingualism: consequences for mind and brain. Trends in cognitive sciences (2012);16(4):240-250

\section{Neuropsychiatric subsyndromes and brain metabolic network dysfunctions in early onset Alzheimer's disease}

D. Perani ${ }^{1}$, T. Ballarini ${ }^{2}$, L. Iaccarino ${ }^{1}$, G. Magnani ${ }^{3}$, N. Ayakta ${ }^{4}$, B. Miller ${ }^{4}$, W. Jagust ${ }^{5}$, M. Gorno-

Tempini ${ }^{4}$

${ }^{1}$ San Raffaele Scientific Institute, Vita-Salute San

Raffaele (Milano)

${ }^{2}$ Division of Neuroscience, San Raffaele Scientific

Institute (Milano)

${ }^{3}$ Department of Neurology, San Raffaele Hospital (Milano)
${ }^{4}$ Department of Neurology, University of California San Francisco (San Francisco-USA)

${ }^{5}$ Helen Wills Neuroscience Institute, University of California San Francisco (Berkeley - USA)

Objectives: Neuropsychiatric symptoms (NPSs) often occur in early-age-of-onset Alzheimer's disease (EOAD) and cluster into sub-syndromes (SSy). The aim of this study was to investigate the association between 18F-FDG-PET regional and connectivity-based brain metabolic dysfunctions and neuropsychiatric SSy.

Materials and methods: NPSs were assessed in 27 EOAD using the Neuropsychiatric Inventory and further clustered into four SSy (apathetic, hyperactivity, affective and psychotic SSy) [1]. 85\% of EOAD showed at least one NPS. Voxel-wise correlations between SSy scores and 18F-FDG PET brain glucose metabolism were measured. Interregional correlation analysis [2] was used to explore metabolic connectivity in the salience (aSN) and default mode networks (DMN) in a larger sample of EOAD $(\mathrm{N}=51)$ and Healthy Controls $(\mathrm{N}=57)$.

Results: The apathetic, hyperactivity and affective SSy were highly prevalent $(>60 \%)$ as compared to the psychotic SSy (33\%). The hyperactivity SSy scores were associated with increase of glucose metabolism in frontal and limbic structures, implicated in behavioral control. A comparable positive correlation with part of the same network was found for the affective SSy scores. The apathetic SSy scores were negatively correlated with metabolism in the bilateral orbitofrontal and dorsolateral frontal cortex known to be involved in motivation and decisionmaking processes.

Discussion: Consistent with these SSy regional correlations with brain metabolic dysfunction, the connectivity analysis showed increases in the aSN and decreases in the DMN.

Conclusion: Behavioral abnormalities in EOAD are associated with specific dysfunctional changes in brain metabolic activity, in particular in the aSN that seems to play a crucial role in NPSs in EOAD [3].

\section{References}

[1] Aalten, P., Verhey, F.R., Boziki, M., Bullock, R., Byrne, E.J., Camus, V., Caputo, M., Collins, D., De Deyn, P.P., Elina, K. and Frisoni, G., Neuropsychiatric syndromes in dementia. Results from the European Alzheimer Disease Consortium. I. Dement Geriatr Cogn Disord (2007);24:457- 463

[2] Lee, D.S., Kang, H., Kim, H., Park, H., Oh, J.S., Lee, J.S. and Lee, M.C., Metabolic connectivity by interregional correlation analysis using statistical parametric mapping (SPM) and 
FDG brain PET; methodological development and patterns of metabolic connectivity in adults. European journal of nuclear medicine and molecular imaging (2008);35(9): 1681-1691

[3] Zhou, J. \& Seeley, W. W. Network dysfunction in Alzheimer's disease and frontotemporal dementia: Implications for psychiatry. Biol. Psychiatry (2014);75:565-573

\section{NEUROPSYCOLOGY AND BEHAVIOUR}

\section{Atypical presentation of AD: a case report}

S. Ciresa, CG. Cerri, C. Perin

Ist Clinici Zucchi GSD, School of Medicine and Surgery UNIMIB (Milano)

Aim: An atypical and rare presentation of $\mathrm{AD}$, characterized by early and prominent executive deficits have been described and named frontal variant of Alzheimer's Disease (fvAD). To our knowledge diagnostic criteria for fvAD have not precisely defined. The cases described in literature are few and report that fvAD should specifically present executive deficits and mild visuospatial and memory impairment. 1 We describe a case of atypical Alzheimer whom early phase of dementia started with executive deficits and neglect-like visuospatial problems.

Materials and Methods: A 66-years-old man righthanded, working as managing consultant, had troubles finding his own car at the end of the working day and decided to walk home for $10 \mathrm{~km}$. Due to this compulsive and "poor judged" behavior, his wife requested a neurological evaluation.

Results: Neurological evaluation showed motor stereotypy and anxiety. Neuropsychological assessment showed a prominent frontal impairment, a slight neglect-like visuospatial deficits and verbal and visuospatial memory deficits. To verify if the patient could have a bvFTD due to prominent frontal and behavioral aspects or a fvAD, a PET was performed that revealed fronto-temporo-parietal lobes hypoperfusion with strong right asymmetry.

Discussion: Patient showed an early dementia compatible with bvFTD. The clinical and neuropsychological features do not meet criteria for bvFTD.2 An atypical presentation of possible AD with early onset of neglect was hypothesized thanks to the clinical assessment. A correct diagnosis is important for the management of the patient, also for the therapeutic implications.

\section{References}

[1] Rascovsky K, Hodges JR, Knopman D, et al. Sensitivity of revised diagnostic criteria for the behavioural variant of frontotemporal dementia Brain. (2011) Sep;134 (Pt 9):2456-77

[2] Habek M, Hajnsek S, Zarković K, Chudy D, Mubrin Z. Frontal variant of Alzheimer's disease: clinico-CSF-pathological correlation. Can J Neurol Sci. (2010) Jan;37(1):118-20

\section{Dr Jekyll and Mr Hyde: the diagnosis disclosure}

A. Ciricugno ${ }^{1}$, S. Basilico ${ }^{1}$, G. Gelosa ${ }^{2}$, T. Ambrogio ${ }^{3}$, G. Bottini ${ }^{1}$

${ }^{1}$ ASST Grande Ospedale Metropolitano Niguarda, University of Pavia (Milano)

${ }^{2}$ ASST Grande Ospedale Metropolitano Niguarda (Milano)

${ }^{3}$ ASST Grande Ospedale Metropolitano Niguarda, University of Milano Bicocca (Milano)

Background: Not only issues in case identification but also ethical and practical questions about disclosure of dementia diagnosis remain subject of debate. Key points in supporting diagnosis disclosure (Practice guidelines) include respect for patient autonomy and the value of truth telling in familial and professional relationship; contrasting positions often cite lack of useful treatments, uncertainty of diagnosis and patient cognitive deficits. Nevertheless psychological aspects regarding patient's prospective are not taken into account in current practice guidelines.

Aims: We describe a case report of a woman, CM, 58 years old, with a family history of dementia, complaining about language disorder.

Methods: At first neurological, radiological and neuropsychological assessments were carried out. Since they were not conclusive, further investigations were suggested (PET imaging and CSF analysis) which patient carried out two years later.

Results: At presentation the patient expressed an urgent desire for diagnosis disclosure showing anxiety and preserved insight; nevertheless she did not follow clinicians' indications and delayed exams after caregiver repeated advice when cognitive symptoms were more severe.

Conclusions: Time gap from presentation to final diagnosis (semantic dementia), was caused by different factors, mainly related to patient's cognitive features, psychological profile and relationships set up between CM and involved professionals. Distinct factors including symptoms awareness, insight, psychological traits and disease features, made 
diagnosis disclosure more subtle and complicated. In conclusion, diagnosis disclosure is a complex process, that must take into account patient sensitivity and care to individual differences in order to promote an optimal approach to it.

\section{Multiple Cognitive Phenotypes in Amyotrophic Lateral Sclerosis}

\author{
M. Consonni' ${ }^{1}$, E. Catricala'², E. Dalla Bella ${ }^{3}$, \\ V. Gessa ${ }^{4}$, G. Lauria ${ }^{3}$, S. Cappa ${ }^{2}$ \\ ${ }^{1}$ IRCCS Carlo Besta Neurological Institute \\ (Milano) \\ ${ }^{2}$ Institute for Advanced Study, IUSS Pavia (Pavia) \\ ${ }^{3} 3$ rd Neurology Unit and Motor Neuron Diseases \\ Center, IRCCS Foundation "Carlo Besta" \\ Neurological Institute (Milano) \\ ${ }^{4}$ Health Department, IRCCS Foundation "Carlo \\ Besta” Neurological Institute (Milano)
}

Objective: The Strong consensus recommendations (2009) proposed behavioural (ALSbi) and/or dysexecutive (ALSci) impairment as the two main clinical profiles of non-motor manifestations in nondemented amyotrophic lateral sclerosis (ALS) patients. We aimed at assessing through a data-driven approach whether clustering pattern of neuropsychological performance can suggest discrete clinical syndromes that progress beyond currently recognized phenotypes.

Methods: We recruited 71 non-demented ALS patients who were submitted to a comprehensive neuropsychological evaluation. Principal component analysis (PCA) was used to identify the clustering pattern of neuropsychological measures. We calculated the occurrence of ALS patients scoring below the normal range on at least two neuropsychological test performances loading on each PCA cluster.

Results: The outcome of PCA demonstrated the existence of three main test clusters. Two were compatible with the recognised ALSbi and ALSci profiles, accounting for $27 \%$ of the patients. An additional third cluster loaded on social cognition, language and memory tests and accounted for $24 \%$ of the patients. Of these, $15 \%$ had a non-executive cognitive impairment, with defective performance on at least two tests belonging to the latter cluster, and were thus unclassifiable according to current criteria.

Conclusion: Our results suggest that language, social cognition and episodic memory deficits can oc- cur as separate and relevant non-motor manifestations in ALS patients, in addition to behavioural anomalies and executive dysfunctions. Further studies are warranted to investigate the neuroanatomic correlations and prognostic value of each phenotype.

\section{Reference}

[1] Strong, M.J., Grace, G.M., Freedman, M., Lomen-Hoerth, C., Woolley, S., Goldstein, L.H., et al. Consensus criteria for the diagnosis of frontotemporal cognitive and behavioural syndromes in amyotrophic lateral sclerosis. Amyotrophic Lateral Sclerosis (2009);10:131-146

\section{Mind and Language as predictor and preventive of dementia. A work proposal}

\author{
G. Dore ${ }^{1}$, M. D’Onofrio ${ }^{2}$ \\ ${ }^{1}$ Freelance professional (Ittiri -SS) \\ ${ }^{2}$ Clinical Neurological, A.O.U. (Sassari)
}

Introduction: Dementia deprives Man of the Conscious thought and its tool, language; is impossible to think without speaking and speech quality essentially reflects the quality of inner narration. The pathology called dementia is a progressive lexical and semantic impoverishment. The discussion will concern the importance of the reality-based way of thought, especially the semantic-verbal aspect, in diagnostics and therapy.

Methods: People of different age, sex and cultural background were observed in speech; precise markers, useful to identify those people at risk of developing dementia. In such cases the way of thought can be completely defined in order to study the risk connected to that way of thinking, as other authors outlined. Standard clinical neurological and neuropsychological cognitive anamnesis was employed, integrated with psychoanalytic-oriented interviews, in accordance with a new therapeutic diagnostic model called psychoneuroanalysis (PNA).

\section{Results: Numerous indicators were revealed:}

- Emotional-egoic exam of Reality, compared to the logic-semantic one.

- Recurrence of paralinguistic and parasemantic stereotypes.

- Predominance of linguistic a holophrastic form.

- Predominance of Pavlovian-thought.

- Etc.

Conclusions: According to the authors, an investigation conducted on the individual way of thought 
on people who are still not involved in any dementigen processes can facilitate neuroscientific research and medical therapy. Therefore, it is easy to understand how important is to improve customised "ante-litteram" unique clinic diagnostics, in order to look for those eco-psychological markers specific and sensitive for Man. The evaluation of results may be useful for a precise rehabilitation treatment "ad personam", in full coherence with the latest findings of Neurosciences.

\section{References}

[1] Dore G. [Un Nuovo paradigma teorico-terapeutico dell'Isteria, esemplificato in un caso clinico di "Distonia" focale in un soggetto di sesso maschile] Tesi specialistica anno 2006-2007, scuola di specializzazione in Neurologia - Università degli studi di Sassari

[2] Dore G., D'Onofrio M. A new model of individual cognitive therapy (cst) for dementia: a pilot study in five cases. European Journal of Neurology (2015);22(1):582-583

\section{The Five Words Test: a rapid screening for the assessment of memory impairment}

G. Gamberini ${ }^{1}$, F.L. Saibene ${ }^{2}$, S. Scioli ${ }^{2}$, A. d'Arma 2 , F. Baglio 2 , R. Nemni ${ }^{3}$ E. Farina ${ }^{2}$

${ }^{1}$ Don C.Gnocchi Onlus Foundation, IRCCS "Santa

Maria Nascente" (Milano)

${ }^{2}$ Don Gnocchi Foundation (Milano)

${ }^{3}$ Don Gnocchi Foundation, University of Milan (Milano)

Background: The differential diagnosis of AD and $\mathrm{MCI}$ is sometimes difficult because the amnesic syndrome can be confused with memory dysfunction due to aging or depression. The Five Words Test (FWT) by Dubois is a screening memory task and its construction aims to identify patients exhibiting memory disorders possibly due to hippocampal dysfunction.

Objectives: To investigate the relationship between an Italian version of FWT (build according to Dubois's instructions) and the most used screening test for cognitive impairment: MMSE.

Method: 60 patients with diagnosis of cognitive impairment (mild AD or MCI): all patients were systematically tested with MMSE (Magni, 1996) and FWT during their first visit.

Results: Statistically significant correlations between FWT (mean score: 7,74, range 0-10) and MMSE (mean: 24,78, range 0-30): FWT/MMSE $(\mathrm{p}=0,0070)$ FWT/MMSE-Recall $(\mathrm{p}=0,0083)$ FWTRecall/MMSE $(\mathrm{p}=0,0208)$ FWT-Learning/MMSERecall ( $\mathrm{p}=0,0022)$

Discussion: These results support the idea that this test can be useful to detect memory dysfunction in people with incoming or early cognitive deterioration. There was no statistically significant correlation between FWT-Learning and MMSE-Learning, probably because the phase of learning in FWT also involves long term memory while in MMSE only involves short term memory. In fact, FWT-Learning correlated with MMSE-Recall.

Conclusion: This is a rapid (about 5 minutes including an interval between learning and recall) test that is easy to use in medical practice to screen patients with probable cognitive impairment. The Italian standardization of the FWT will be completed soon.

\section{Reference}

[1] Dubois B., Touchon J., Portet F., Ousset P.J.; Vellas B., Michel B. "The 5 words": a simple and sensitive test for the diagnosis of Alzheimer's disease. Press Med (2002) Nov 9;31(36):1696-9

\section{Predicting the reading profiles of different neurodegenerative impairments: The case of Alzheimer's disease, Progressive Primary Aphasia and Posterior Cortical Atrophy}

\author{
C. Luzzatti ${ }^{1}$, E. Ripamonti ${ }^{2}$, G. Lazzati ${ }^{1}$, \\ E. Martini ${ }^{3}$, F. Lucchelli ${ }^{3}$ \\ ${ }^{1}$ Department of Psychology, University of Milan- \\ Bicocca (Milano) \\ ${ }^{2}$ Department of Economics, Management \& \\ Statistics, Milan Center for Neuroscience, \\ University of Milan-Bicocca (Milano) \\ ${ }^{3}$ Passirana - G. Salvini Hospital (Rho - MI)
}

Objectives: We aimed at testing the reading performance in a sample of patients with neurodegenerative impairment.

Materials: We used two reading tasks of words and nonwords, and of words with unpredictable stress position, a repetition task of words and nonwords, and two lexical decision tasks (written and oral modalities).

Methods: We tested patients with Alzheimer's disease ( $A D, n=19)$, Primary Progressive Aphasia (PPA, $n=6$, three logopenic variants, one semantic 
variant and one pure anomic) and Posterior Cortical Atrophy (PCA, n=4). We also tested 34 healthy participants as a control group.

Results: In oral lexical decision AD patients had significantly lower performance than those with PCA, whereas the opposite pattern was found in written lexical decision. Reading of nonwords was more impaired than reading of words in both $A D$ and PPA patients. Multiple single case analysis identified phonological dyslexia in $50 \%$ of PCA cases, in a much lower percentage in patients with $\mathrm{AD}(15.8 \%)$ and PPA (16.7\%). In one AD patient the reading disorder was classified as surface dyslexia.

Discussion: No specific patterns of reading performance emerged as predictors of different neurodegenerative impairments. In general, in written lexical decision PCA patients show a worse performance as compared to that obtained in the other two groups. On the contrary, worse performance in the oral modality may orient towards a diagnosis of AD.

Conclusions: Various forms of neurodegenerative disease occur with different cognitive profiles, and reading processes are not an exception to this rule.

\section{References}

[1] Brambati, S. M., Ogar, J., Neuhaus, J., Miller, B. L., \& Gorno-Tempini, M. L. Reading disorders in primary progressive aphasia: a behavioral and neuroimaging study. Neuropsychologia (2009);47:1893-1900

[2] Henry, M. L., Beeson, P. M., Alexander, G. E., \& Rapcsak, S. Z. Written language impairments in primary progressive aphasia: a reflection of damage to central semantic and phonological processes. Journal of Cognitive Neuroscience (2012);24:261-275

[3] Strain, E., Patterson, K., Graham, N., \& Hodges, J. R. Word reading in Alzheimer's disease: cross-sectional and longitudinal analyses of response time and accuracy data. Neuropsychologia (1998);36:155-171

\section{Frailty and cognitive decline: the role of metacognitive-executive dysfunction}

\author{
S. Palermo ${ }^{1}$, I. Rainero ${ }^{1}$, E. Rubino ${ }^{1}$, M. Zucca $^{1}$, \\ M. Amanzio ${ }^{2}$ \\ ${ }^{1}$ Department of Neuroscience, University of Turin \\ (Torino) \\ ${ }^{2}$ Department of Psychology, University of Turin \\ (Torino)
}

Aims: Frailty increases the risk of future cognitive decline, while cognitive impairment increases the risk of frailty, suggesting that cognition and frailty interact within a cycle of decline associated with aging $[1,2]$. The aim of the current study is to investigate whether frailty could be influenced by cognitive-behavioral measures and executive dysfunction, such as action-monitoring.

Material: 60 consecutive subjects $(\mathrm{M} / \mathrm{F}=22 / 38$; mean age $\pm \mathrm{SD}=69.6 \pm 6.8$ years) complaining of different cognitive deficits and presenting for the first time at the out-patient service of our dementia clinic were involved. Frailty was assessed using the Multidimensional Prognostic Index (MPI [3]), in line with a bio-psycho-social integrated approach.

Methods: To study whether MPI could be influenced by cognitive dysfunction, we conducted three multiple linear regression analyses adjusted for a combination of neuropsychological-behavioral measures.

Results: The relationship among MPI, action monitoring, depression and disinhibition, were highly significant ( $p=0.02, p=0.004, p=0.0006$, respectively). We also found a relationship between MPI and a reduced awareness of autonomy in daily living in terms of instrumental activities $(\mathrm{p}=0.02)$.

Discussion: Our data support the novel notion that frailty is related with metacognitive-executive dysfunction. Frailty may be due to a possible dysfunction of the medial prefrontal-ventral striatal network, observed through our patients monitoring disability, mood changes and reduced awareness of iADL.

Conclusions: The study of frailty and its neuropsychological correlates is clinically important because this phenomenon may have diagnostic, nosological, and prognostic values that affect patients' wellbeing from MCI to early dementia stages.

\section{References}

[1] Canevelli M., Cesari M., van Kan G.A. Frailty and cognitive decline: how do they relate? Curr Opin Clin Nutr Metab Care (2015);18:43-50

[2] Robertson D.A., Savva G.M., Kenny R.A. Frailty and cognitive impairment. A review of the evidence and causal mechanisms. Ageing Res Rev (2013);12:840-851

[3] Pilotto A., Sancarlo D., Panza F., Paris F., D’Onofrio G., Cascavilla L., Addante F., Seripa D., Solfrizzi V., Dallapiccola B., Franceschi M., Ferrucci L. The Multidimensional Prognostic Index (MPI), based on a comprehensive geriatric assessment predicts short- and long-term mortality in hospitalized older patients with dementia. J Alzheimers Dis (2009);18(1):191-9 


\section{"Progetto Dina Scalise" an intergenerational program to prevent cognitive impairment}

\author{
A. Scalise \\ University Hospital of Udine (Udine)
}

Objectives: As the worldwide population ages, dementia has reached epidemic proportions, with social, medical and economical burdens. While living alone, having feelings of loneliness, depression, social isolation and psychosocial stress can increase dementia, engaging in exercise, and cognitively and socially stimulating activities are protective.

Methods: The "Progetto Dina Scalise" was planned and implemented as health promotion program that utilizes an intergenerational engagement approach to respond to such needs, while simultaneously enabling the elders to contribute to society and younger generations. From 2014 to date the project has involved 77 subjects older than 65 y.o. who volunteered their time and experience in local schools, to share moments of life, school experiences, stories, games with children. Data from questionnaires (MoCA, PSQI, EQ5-D, GDS) are collected at baseline and each subsequent year.

Results: The "Progetto Dina Scalise" has shown improvement in physical and psychological functioning of elders and in healthy upbringing of children.

Discussion: The findings suggest that it is important that the elders maintain their social role and engage in intellectual activities to help preserve their physical and psychological health. The project, a multimodal lifestyle-related preventive strategy, has many components for elderly, such as promoting their health, as well as supporting the healthy upbringing of children against the ageism.

Conclusion: Health promotion efforts for elderly must support social policy for the creation of a meaningful service programs for elderly on a large social scale. As such, an intergenerational program is an effective and sustainable program for health promotion among elderly.

\section{References}

[1] Yasunaga M, Murayama Y, Takahashi T, Ohba H, Suzuki H, Nonaka K, Kuraoka M, Sakurai R, Nishi M, Sakuma N, Kobayashi E, Shinkai S, Fujiwara Y. Multiple impacts of an intergenerational program in Japan: Evidence from the Research on Productivity through Intergenerational Sympathy Project. Geriatr Gerontol Int. (2016) Mar;16 Suppl 1:98-109. doi:10.1111/ggi.12770.
[2] Morita K, Kobayashi M. Interactive programs with preschool children bring smiles and conversation to older adults: timesampling study. BMC Geriatr.(2013) Oct 18;13:111. doi: 10.1186/1471-2318-13-111

[3] Sindi S, Mangialasche F, Kivipelto M. Advances in the prevention of Alzheimer's Disease. F1000Prime Rep. (2015) May 12;7:50. doi: 10.12703/P7-50

\section{Hypoxia impairs brain prefrontal lateralization related to visuospatial orienting of attention as reflected by an ERPs CNV neuromarker}

\author{
A. Zani, S. Porcelli' ${ }^{1}$, M. Marzorati ${ }^{1}$, G. Marsili², \\ A. Senerchia ${ }^{2}$, M. Bertoli², E. Rizzi1 ${ }^{1,2,3}$, A. M. \\ Proverbio $^{2,3}$ \\ ${ }^{1}$ Institute of Molecular Bioimaging and Physiology, \\ National Research Council (CNR IBFM) (Segrate- \\ MI) \\ ${ }^{2}$ Department of Psychology, University of Milan- \\ Bicocca (Milano) \\ ${ }^{3}$ Milan Center for Neuroscience (NEUROMI), \\ University of Milan-Bicocca (Milano)
}

Aims: Brain oxygen requirements are among the highest of all biological organs, thus brain sensitivity to hypoxia is heavy. Indeed, much knowledge is available about the consequences of severe and nearlethal hypoxia on brain anatomy and physiology. By contrast, the impact of non-pathological hypoxia on neurocognitive processing and behaviors still remains relatively unexplored and controversial. The present study assessed alterations of brain functionality during a demanding yet structurally-not-hurtful hypoxia focusing on brain hemispheric processing specificity in alerting and spatial orienting of visual attention as reflected by an event-related contingent negative variation (CNV) neuromarker.

Materials and Methods: We recorded high spatialdensity ERPs in two separate sessions in which healthy volunteers breathed either ambient-air or oxygen-impoverished $(\sim 12.5 \%)$ air. During each session, three cue-target tasks eliciting either a phasic or a tonic alerting as well as a visuospatial orienting of attention were presented.

Results: Prior targets presentation ERP waveforms showed a CNV with a maximum at prefrontal sites as indicated by topographical maps. In normoxia this variation was larger over the left as compared to the right hemisphere for orienting of attention but not for both phasic and tonic alerting. Most importantly, overall hypoxia boosted CNV but disrupted the orienting-related functional lateralization observed in normoxia. 
Discussion and Conclusions: Besides pointing at the CNV as a robust neuromarker of prefrontal processing lateralization associated with spatial orienting of visual attention, our findings indicate that a demanding yet not-hurtful hypoxia impairs this at- tention orienting-related lateralization. This hints at possible affinities with pathological alterations underpinning attentional deficits in specific neuropsychological patients. 


\section{CLINICAL MANAGEMENT}

\section{Human perception of image complexity: real scenes versus texture patches}

\author{
S. E. Corchs, G. Ciocca, F. Gasparini \\ Department of Informatics, Systems and \\ Communication, University of Milano Bicocca \\ (Milano)
}

The aim of this work is to study image complexity perception of real images. We conducted psychophysical experiments where observers judged the complexity of different datasets of images on a webbased interface [1]. At the end of the test, observers indicated the main characteristics that guided their judgements. The databases differed in the type of visual stimuli used: images representing real scenes and/or texture patches. For real scenes the most relevant criteria used were quantity of objects, details and colors, while for texture patches they were regularity and understandability. Several criteria are adopted simultaneously, confirming the multidimensional aspect of complexity found in the literature [2]. To process the subjective data we applied $\mathrm{z}$ scores and outlier removal. The mean scores are then correlated with different visual features. We considered features based on spatial, color and frequency properties that can be associated to bottom-up processes. To take into account top-down effects like understandability we included a memorability index [3]. We propose an image complexity measure where the features are linearly combined. The optimal weighting coefficients are those that best fit the subjective data and depend on the type of stimuli considered. Our measure, properly tuned, can predict complexity perception of different kind of images, outperforming the single visual features. From our investigation two aspects of image complexity can be underlined: many different perceptual properties are involved and their relative influence depends on the type of stimuli. These considerations are supported by both our computational proposal and the verbal description analysis.

\section{References}

[1] Ciocca G, Corchs S, Gasparini F, Bricolo E, Tebano R. Does color influence image complexity perception? In: Fifth IAPR
Computational Color Imaging Workshop vol. 9016 of Lecture Notes in Computer Science. Springer Berlin/Heidelberg; (2015):139-148

[2] Oliva A, Mack ML, Shrestha M. Identifying the Perceptual Dimensions of Visual Complexity of Scenes. In: Proc. 26th Annual Meeting of the Cognitive Science Society (2004):101-106

[3] Isola P, Xiao J, Torralba A, and Oliva A. What makes an image memorable? In IEEE Conference on Computer Vision and Pattern Recognition (2011):145-152

\section{Conversational analysis in the differential diagnosis between cognitive impairment and functional cognitive impairment (depression)}

\author{
R. Meroni ${ }^{1}$, I. Appollonio², M. Beghi², C. Perin ${ }^{2}$, \\ F. Peroni' ${ }^{2}$, M. Polita ${ }^{3}$, B. Viganò ${ }^{2}$, C. Cornaggia ${ }^{2}$ \\ ${ }^{1}$ Istituti Clinici Zucchi, GSD (Carate Brianza-MB) \\ ${ }^{2}$ School of Medicine and Surgery, University of \\ Milan Bicocca (Monza) \\ ${ }^{3}$ Department of Literary Studies, Philology and \\ Linguistics, University of Milan (Milano)
}

Aim: In neurology, the differential diagnosis between cognitive and functional cognitive impairment is one of the most complex and difficult especially in early stages of the disease. Conversation analysis (CA) study social interaction embracing both verbal and non-verbal conduct in situations of everyday life. Our study aims to evaluate the possibility of using linguistic analysis to support clinical, and test-based diagnoses for the differential diagnosis between cognitive impairment and psychiatric diagnosis resembling dementia.

Methods:13 subjects requesting an initial consultation in a Alzheimer Evaluation Unit. A provisional diagnosis through a neuropsychological evaluation, was made at the baseline, after six months (gold standard), and, if necessary, after 12 months. The linguistic analysis was performed in a blind study by a linguist at T0. Patients language was studied at linguistic and conversational level.

Results: Linguistic analysis at T0 has shown a correspondence rate with the gold standard diagnosis in $76.9 \%$ of cases (sensibility- $80 \%$, specificity- $75 \%$, PPV-67\% NPV-86\%). The correspondence rate between the neuropsychological équipe analyses at $\mathrm{T} 0$ with the gold standard was $58.4 \%$. 
Discussion: The linguistic diagnosis was able to establish the presence of cognitive impairment earlier than the diagnosis made by the neuropsychological équipe. A linguistic cluster of patognomonic phenomenon can lead to a diagnosis with a good degree of reliability, thus a trained linguist is needed.

Conclusion: New studies using this methodology, and on larger patient populations are needed in order to confirm the use of this technique and, if necessary, to refine it with the addition of words linked to emotions.

\section{Effects of dynamic tilt-table with integrated robotic stepping associated with functional electrical stimulation in acute stroke: a pilot study}

\author{
D. Piscitelli ${ }^{1}$, L. Pelliccari ${ }^{2}$, R. Meroni ${ }^{1}$, D. Ruma ${ }^{3}$, \\ C. Cerri ${ }^{1}$, E. Tallarita ${ }^{3}$ \\ ${ }^{1}$ School of Medicine and Surgery, Program in \\ Physical Therapy, University of Milan Bicocca \\ (Carate Brianza-MB) \\ ${ }^{2} P h D$ Program in Advanced Sciences and \\ Technologies in Rehabilitation Medicine and \\ Sports, Tor Vergata University (Roma) \\ ${ }^{3}$ Rehabilitation Department, S. Anna Hospital \\ (Como)
}

Objective: The aim of this study was to investigate the short-term effects of orthostatic training on a robotic-system in patients with acquired brain injury (ABI).

Materials: Two patients (age 70,5 $\pm 6,7$ years; $38,5 \pm 5$ days/post-stroke) with $\mathrm{ABI}$ were subjected to an orthostatic training using a dynamic tilt-table with integrated robotic-stepping [1], combined with a step-synchronized functional-electrical stimulation (FES) to assist leg movements [2,3].

Methods: Eleven 30-minutes treatments were performed, with and without FES on alternate days. The treatment (in addition to conventional physiotherapy) involved a tilting to $30^{\circ}$ in the first 5-minutes, increasing to a maximum of $80^{\circ}$ according to patient's clinical condition. Blood pressure (BP), heart rate (HR) and oximetry were recorded at the baseline and every 5-minutes during treatment. Muscle tone was assessed before and after each session using the Modified-Ashworth scale (MAS).

Results: Subjects completed 10 and 5 sessions, showing an improvement of clinical measures (BP: form $122 \pm 5 / 86 \pm 3$ to $132 \pm 2 / 89 \pm 1 \mathrm{mmHg}$, systolic/ diastolic; HR: from $90 \pm 4$ to $86 \pm 2$ beats/min), furthermore muscle tone decreased in the lower limbs (from $1+$ to $0, \mathrm{MAS}$ ). There was no difference between treatment with and without FES.

Discussion: Patients showed an improvement of salient vital parameters; the amount of incomplete training was consistent with previous studies [3].

Conclusion: Dynamic tilt-table training may improve the orthostatic tolerance and decrease spasticity in the short term period. Our results seem promising, further studies are needed to determine whether these findings are generalizable to all $\mathrm{ABI}$ patients and if the results remain stable over time.

\section{References}

[1] Chang WH, Kim YH. Robot-assisted Therapy in Stroke Rehabilitation. J Stroke. (2013);15:174-81

[2] Wieser M, Gisler S, Sarabadani A, Ruest RM, Buetler L, Vallery H, Klamroth-Marganska V, Hund-Georgiadis M, Felder M, Schoenberger JL, Gutknecht C, Riener R. Cardiovascular control and stabilization via inclination and mobilization during bed rest. Med Biol Eng Comput. (2014);52:53-64

[3] Luther MS, Krewer C, Muller F, Koenig E. Comparison of orthostatic reactions of patients still unconscious within the first three months of brain injury on a tilt table with and without integrated stepping. A prospective, randomized crossover pilot trial. Clin Rehabil. (2008);22:1034-41

\section{Design of a protocol for osteopathic manipulative treatment for frequent episodic tension-type headache (EBOTTH)}

L. Tremolizzo', M. Gioda ${ }^{2}$, F. Somalvico ${ }^{3}$,

A. Maggiani ${ }^{4}$, L. Papa $^{5}$, F. Spaziante ${ }^{6}$, M. Roppolo ${ }^{2}$, G. Tornatore ${ }^{6}, \mathrm{G}$. Rolle ${ }^{2}$

${ }^{1}$ School of Medicine and NeuroMI, University of

Milano-Bicocca (Monza)

${ }^{2}$ Research Department, Italian School of

Osteopathy and Manual Therapies (SIOTEMA)

(Sartirana - PV)

${ }^{3}$ Research Department, Alpha Search (Milano)

${ }^{4}$ Research Department, Italian Academy of

Osteopathic Medicine (AIMO) (Saronno-VA)

${ }^{5}$ Research Department, European Research Center of Osteopathic Medicine (Milano)

${ }^{6}$ Research Department, Take Care Osteopathic Institute (Milano)

Introduction: Frequent episodic tension-type headache (ETTH) is the most common type of primary headache disorder encountered in clinical practice. The high prevalence of ETTH, considering 
its individual impact and huge financial costs, has important implications for health policy, since it is a source of high but potentially reducible socioeconomic burden. Often patients do not respond completely to drug prophylaxis and turn to complementary or alternative therapies, such as osteopathic manipulative treatment (OMT). Our pilot study (Rolle et al. 2014) showed the efficacy of OMT in reducing headache frequency in ETTH patients.

Material and Methods: This is a protocol for a multicentre, double-blind, randomized, sham-controlled trial (RCT) exploring, with full power, the efficacy of OMT in ETTH. Sample size has been estimated in 264 patients. Treatments will be performed during the first 30 days following enrolment.

Results: Primary outcome will be the change at 120 days in patient-reported headache frequency of at least $30 \%$ with respect to the control group. Secondary outcomes will include the assessment of changes in: headache intensity, symptomatic drug use, HDI, HIT-6, Goal Attainment Scaling for somatic dysfunction, side effects.

Conclusions: If OMT effectiveness could be demonstrated, we might expect two putative repercussions in ETTH management: (a) increased compliance; (b) lower rate of side effects. Furthermore, OMT would increase the range of available strategies for managing ETTH in clinical practice. https://www.clinicaltrials.gov/ct2/show/ NCT02592850?term=ebotth\&rank=1

\section{Reference}

[1] Rolle G, Tremolizzo L, Somalvico F, Ferrarese C, Bressan LC. Pilot trial of osteopathic manipulative therapy for patients with frequent episodic tension-type headache. J Am Osteopath Assoc. (2014);114:678-85

\section{Sport Therapy and Osteopathy Manipulative Treatment in ALS (ME_E_SLA)}

\author{
L. Tremolizzo ${ }^{1}$, A. Ferri ${ }^{2}$, G. Corna ${ }^{1}$, C. Lunetta ${ }^{3}$, \\ V. Silani ${ }^{4}$, N. Riva ${ }^{5}$, A. Rigamonti ${ }^{5}$, A. Maggiani ${ }^{6}$, \\ C. Ferrarese ${ }^{1}$, F. Lanfranconi ${ }^{1}$, MOTOR study \\ group $^{7}$ \\ ${ }^{1}$ School of Medicine and NeuroMI, University of \\ Milano-Bicocca (Monza) \\ ${ }^{2}$ Clinical Exercise Science Research Program, \\ Institute of Sport, Exercise and Active Living \\ (ISEAL), Victoria University (Melbourne-AUS)
}

${ }^{3}$ NEuroMuscular Omnicentre (NEMO), Fondazione Serena Onlus (Milano)

${ }^{4}$ IRCCS Istituto Auxologico Italiano, University of Milan (Milano)

${ }^{5}$ Neurology, San Raffaele Hospital (Milano)

${ }^{6}$ Research Department, Italian Academy of

Osteopathic Medicine (AIMO) (Saronno-VA)

${ }^{7}$ Study group - collaborators are listed separately

(Monza)

Introduction: Albeit physical therapy represents one fundamental option recommended for amyotrophic lateral sclerosis (ALS), the exact modalities by which administering this intervention have not yet been explored extensively.

Methods: This project assessed the impact of individualized cardiovascular and strength training (active study arm: TRAIN) in ALS patients. Muscle oxidative metabolism was studied by near-infrared spectroscopy (NIRS). The investigators also assessed the effects of training on quality of life (QoL). Patients were assessed before (time T0) and after three months (time T1). After one month of de-training (time T2) the hypothetic persistence of any treatment-related effect was evaluated. The effect of osteopathic manipulative treatment (passive study arm: OMT) on QoL and pain was studied on a separate group of patients.

Results: ALS patients displayed on average 50\% lower VO2peak values, paralleled by significantly lower muscle oxygen extraction capacity (on average $60 \%$ ). A correlation between these variables was found in ALS patients but no in CTRL. A residual tolerance to exercise was found for each patient, allowing a specific training program to be designed: a tendency toward better functional neurological outcome was shown in the TRAIN group; no harmful effect of exercise was documented. In the OMT arm, the intervention was demonstrated feasible and safe and patients displayed high satisfaction. Considering QoL and pain no significant differences emerged.

Conclusions: Both active training and OMT are feasible options in ALS in controlled settings. More data are necessary before concluding on the impact of these interventions in ALS patients. https://clinicaltrials.gov/ct2/show/NCT02548663 


\section{GENETICS}

\section{Glioblastoma multiforme: a multidisciplinary approach to overcome chemoresistance and find new therapeutic strategies}

G. Riva, C. Negroni, C. Cilibrasi, G. Romano,

S. Redaelli, C. Giussani, R. Giovannoni,

A. Bentivegna

School of Medicine and Surgery, University of Milano-Bicocca (Monza)

Objectives: Glioblastoma multiforme is the most frequent malignant brain tumor. Patients die within 15 months after diagnosis. The failure of current therapies is ascribed to a subpopulation of cells with stem-like properties, called glioma stem cells (GSCs). The aim of this study is to develop new effective therapies. Moreover, we want to better characterize the orthotopic xenograft model established by GSCs injection into NOD/SCID mice.

Materials and methods: We tested Temolomide and Valproic acid treatments, alone and in combination, on seven GSC lines by MTT assay and we sequenced p53. Moreover, we characterized our xenograft model investigating the expression of stemness and differentiation markers by immunohistochemistry on FFPE tissues and by immunofluorescence on the correspondent cell line. Finally, we performed aCGH on the DNA extracted from the cell line and from FFPE tissues.

Results: GSCs were resistant to Temozolomide and slightly sensitive to Valproic acid. The two drugs exerted a synergistic effect when combined performing a pre-conditioning with Valproic acid. Furthermore, several cell lines carry p53 mutations. IF and IHC showed a perfect correspondence for stemness markers expression, but discordant data for the others. aCGH analysis evidenced numerous alterations specific for the ex vivo sample, suggesting the presence of an in vivo clonal selection.

Discussion: This work shows the importance of murine microenvironment in GSCs phenotype in vivo and suggests the possibility to use our combined treatment for therapeutic purposes.

Conclusions: Orthotopic models from GSCs and in vitro grown cell lines represent good models for the development of GSC-targeted therapies.

\section{NEUROBIOLOGY}

\section{Translational approach to chemotherapy induced peripheral neurotoxicity (CIPN) - neurophysiology as the link between bench and bed-side}

\author{
P. Alberti, G. Fumagalli, G. Cavaletti \\ Experimental Neurology Unit, University of \\ Milano-Bicocca (Monza)
}

Objective: Chemotherapy Induced Peripheral Neurotoxicity (CIPN) burdens a large cancer survivor population. No cure is avalaible. Translationability should be implemented. Neurophysiology might be the answer: it is applicable in animals and in humans. Our aim was following a standardised neurophysiological protocol in animals, similar to clinical practice.

Materials and Methods: 20 male BALB/C mice were tested. Sensory nerve conduction study of caudal and digital nerves, motor nerve conduction studies of sciatic nerve, F-waves of sciatic nerve were obtained. All determinations were performed under standard conditions in a temperature-controlled room; deep isofluorane anaesthesia was performed.

Results: Mean sensory action potential (SAP) amplitude and sensory conduction velocity (SCV) for caudal nerve were respectively $80,82 \mathrm{uV}$ (sd:9,95) and $31,33 \mathrm{~m} / \mathrm{sec}$ (sd.:1,63); mean SAP amplitude and SCV for digital nerve were respectively 91,73 $\mathrm{uV}(\mathrm{sd}: 26,10)$ and $26,61 \mathrm{~m} / \mathrm{sec}(\mathrm{ds}: 1,21)$; mean sciatic distal compound muscle action potential (CMAP) amplitude and motor conduction velocity (MCV) were respectively 55,05 $\mathrm{mV}(\mathrm{sd}: 13,35)$ and $46,37 \mathrm{~m} / \mathrm{sec}$ (sd:2,91); mean F waves latency for sciatic nerve was $7,18 \mathrm{msec}$ (sd:1,08).

Discussion: All variables were obtained easily and rapidly following our protocol. The key issue was recording adequate amplitude for sensory nerves. CIPN is mainly a sensory length-dependent axonal neuropathy/neuronopathy. Thus, SAP amplitude could be the best parameter to verify neuroprotectan efficacy. This object was fullfilled. Moreover, a set of reference values for all recordings in animals was defined.

Conclusion: An algorithm for an extensive neurophysiological testing in animals could be proposed to transfer preclinical data to CIPN preventive clinical trials. 
MEF2C and MEF2D alterations in sporadic and familial ALS patients: a possible correlation with disease progression?

A. Arosio ${ }^{1}$, G. Sala ${ }^{1}$, V. Rodriguez-Menendez ${ }^{1}$,

D. Grana ${ }^{2}$, F. Gerardi ${ }^{3}$, C. Lunetta ${ }^{3}$, C. Ferrarese ${ }^{1}$, L. Tremolizzo ${ }^{1}$

${ }^{1}$ School of Medicine and Surgery and Milan Center for Neuroscience (NeuroMI), University of MilanoBicocca (Monza)

${ }^{2} \mathrm{PhD}$ program in Neuroscience, School of Medicine and Surgery and Milan Center for Neuroscience (NeuroMI), University of Milano-Bicocca (Monza) ${ }^{3}$ NEuroMuscular Omnicentre (NEMO), Fondazione Serena Onlus (Milano)

Objectives: Amyotrophic Lateral Sclerosis (ALS) is a lethal neurodegenerative disease affecting motor neurons very difficult to diagnose. For this reason, the identification of biomarkers useful to identify and to monitor the disease is an urgent goal. Since ALS is a neuromuscular disease, we assessed in accessible biofluids the potential involvement of two members of Myocyte Enhancer Factor 2 family (MEF2), a group of transcriptional factors regulating many important functions in central nervous system and muscle development and maintenance.

Materials: Peripheral Blood Mononuclear cells (PBMCs) obtained from 30 sporadic ALS patients (sALS), 9 subjects with SOD1 gene mutations (SOD1+) and 30 healthy controls (CTRL) were used for the analysis.

Method: PBMCs isolation; Real-time PCR (qPCR); Western blotting (WB); Immunofluorescence.

Results: We observed increased MEF2C and MEF2D mRNA levels in both sALS and SOD1+ patients with respect to controls. MEF2 altered intracellular localization and function were also reported in patient cells. Moreover, in 8 sALS patients MEF2C and MEF2D mRNA levels were re-evaluated at the follow-up showing a significant correlation with the disease progression index (DPI).

Discussion: MEF2C and MEF2D altered expressions were reported in circulating cells of patients suggesting the opportunity to test these parameters as possible biomarkers in ALS. Furthermore, preliminary results evidenced that MEF2 expression over time might be different between fast and slow progression patients.
Conclusions: A dedicated study will be planned to explore the eligibility of MEF2 as state marker of disease confirming previous results reporting that MEF2C mRNA levels negatively correlated with longevity in ALS animal model.

\section{References}

[1] Arosio, A., Sala, G., Rodriguez-Menendez, V., Grana, D., Gerardi, F., Lunetta, C., Ferrarese, C., Tremolizzo, L., MEF2D and $\mathrm{MEF} 2 \mathrm{C}$ pathways disruption in sporadic and familial ALS patients. Mol Cell Neurosci. (2016);74:10-17

[2] Calvo, A.C., Manzano, R., Atencia-Cibreiro, G., Oliván, S., Muñoz, M.J., Zaragoza, P., Cordero-Vázquez, P., EstebanPérez, J., García-Redondo, A., Osta, R., Genetic biomarkers for ALS disease in transgenic SOD1(G93A) mice. PLoS One (2012); 7:e32632

\section{In vitro activity and neurotoxicity of new promising metal-based anticancer complexes}

C. Ceresa ${ }^{1}$, G. Nicolini ${ }^{1}$, S. Semperboni ${ }^{1}$, M. Pellei ${ }^{2}$, N. Margiotta ${ }^{3}$, V. Gandin ${ }^{4}$, J. Hoeschele ${ }^{5}$, C. Santini ${ }^{2}$, G. Cavaletti ${ }^{1}$

${ }^{1}$ Experimental Neurology Unit, School of Medicine and Surgery, University of Milano-Bicocca (Monza)

${ }^{2}$ School of Science and Technology-Chemistry Division, University of Camerino (Camerino-MC)

${ }^{3}$ Department of Chemistry, University of Bari A. Moro (Bari)

${ }^{4}$ Department of Pharmaceutical and Pharmacological Sciences, University of Padova (Padova)

${ }^{5}$ Department of Chemistry, Eastern Michigan University (Ypsilanti - USA)

The approved platinum drugs continue to have a major role in cancer treatment. However, despite their efficacy, serious side effects often prevent their administration at full efficacious doses or may considerably affect patients' quality of life. Hence, there is an urgent need to find safer and better-tolerated chemotherapeutic drugs. In this work we investigated in vitro the activity and the neurotoxicity of new anticancer complexes based on copper ([Cu(PTA)4] PF6; [Cu(thp)4]PF6), gold ([Au(PTA)4]PF6) and platinum ([PtCl2(cis-1,4-DACH)]; [Pt(1,1'-CBDCA)(cis-1,4-DACH)]). Cytotoxicity was tested by MTT assay in a panel of human cancer cells. Neurotoxicity was evaluated by an in vitro model based on organotypic cultures of DRG from E15 rat embryos. 
Since the ubiquitin-proteasome system is a cancer cell molecular target of copper and gold baseddrugs, we evaluated, by fluorimetric assay, their ability to hinder the proteasome machinery in DRG neurons. At 48 hours, both copper compounds were not neurotoxic even at higher concentrations with respect to the IC50 calculated in cancer cells while [Au(PTA)4]PF6 was neurotoxic at lower concentration than IC50. [PtCl2(cis-1,4-DACH)] elicited a neurotoxicity slightly lower with respect to oxaliplatin. Conversely, [Pt(1,1'-CBDCA)(cis-1,4-DACH)] showed a reduced neurotoxicity compared with the reference drug. Both copper-based compounds, that are not neurotoxic, do not inhibit proteasome activity in DRG neurons. Contrarily, the neurotoxic complex [Au(PTA)4]PF6, induces a significant inhibition of proteasome activity. Our results, together with the low IC50 of the copper and platinum based complexes, suggest them as promising compounds providing support to further in vivo studies.

\section{Cracking the Neural Code - A Gedanken Experiment: "The Alien Task"}

\section{Cerizza \\ Department of Neurology, Bicocca University, Monza Hospital (Monza)}

The concept of Spike is central in actual models of neural coding and the mean firing rate has been widely analyzed by information theory too as its most prominent aspect. Nevertheless, both computational models and clinical neurophysiology, are still lacking of an effective micro neurophysical theory for the underlying phenomena. A Gedanken-experiment might describe our actual knowledge of neural code as an "Alien-Task": "Alien scientists of a remote interstellar planet have been studying with their telescopes for a couple of centuries, the human knowledge transmission system in a primary school. Due to the perpendicular intergalactic perspective they ignore the existence of written signs and words on the vertical blackboard of the school. They have a well-established theory correlating the mean frequency of school chalkboard erasures, over years, months, days and hours with fluxes and progression of pupils from one class to the other, till the final school exit. As humans we know although that chalkboard erasure itself is meant simply as a tool to give space to new or updated information. A wellkept secret for aliens." The crucial point is that the spike itself is not the only carrier of information but, a sort of refresh system for the wired neuronal cell and and a fortifyer, of environmental field shaped wireless informations.The continuous flux of elisional events, occurring across neural membranes, gives the way to a sub microscopic field of informations, which is wired ,fortifyied and timed by the well-known events called spikes, themselves macroelisional events.

\section{References}

[1] Selman, B. et al. Challenge Problems for Artificial Intelligence. Proceedings of AAAI-96, National Conference on Aritifical Intelligence, AAAI Press, 1996

[2] Wen Ran Zhang YinYang bipolar dynamic logic (BDL) and equilibrium-based computational neuroscience. International Joint Conference on Neural Networks (2009); 3534-3541.

[3] John R. Searle The Mystery of Consciousness New York Review of Books, 1997

\section{Effects of neoglycosylated collagen matrices on neuroblastoma and human stem cells: a new perspective for neuro- regeneration?}

L. Cipolla ${ }^{1}$, L. Russo ${ }^{1}$, A. Sgambato ${ }^{1}$, R. Guizzardi ${ }^{1}$, M. Lecchi ${ }^{1}$, V. Pastori ${ }^{1}$, L. Petecchia ${ }^{2}$, P. Gavazzo ${ }^{2}$, M. Vassalli ${ }^{2}$

${ }^{1}$ Dept. of Biotechnology and Biosciences, University of Milano-Bicocca (Milano)

${ }^{2}$ Institute of Biophysics, National Research Council (Genova)

Scope of the work: Regenerative medicine approaches based on scaffolds obtained form extracellular matrix (ECM) proteins are rapidly expanding. These scaffolds can be bioactivated with signalling cues in order to maintain cell viability and to control and guide cell behaviour. Among signalling molecules, carbohydrates play a key role. Given these premises, the scope of this work has been the evaluation of the effect of neoglycosylated collagen matrices on the behaviour of F11 neuroblastoma cell line, human umbilical cord blood- and bone marrow-derived mesenchymal stem cells in terms of viability and differentiation potential towards functionally active neuronal cells.

Materials and Methods: Collagen Type I from bovine Achilles tendon matrices were functionalised by reductive amination with maltose, lactose, cellobiose, 3'-sialyllactose, 3'-sialyllactose [1-3] leading to a covalent stable neoglycosylation pattern on the 
matrices. F11, hUCB-MSC and hBM-MSC cells were cultured on the differently neoglycosylated collagen matrices.

Results and Discussion: When F11 cells were cultured on collagen matrices functionalised with glucose showed neuritic-like processes, the presence of late neuronal marker $\beta$-tubulinIII and characteristic neuronal electrical activity. Neoglucosylated collagen was also tested with hUCB-MSC (umbilical cord) and hBM-MSC (bone marrow). Both cell lineages cultured on neoglucosylated collagen showed the presence of Nissl bodies (indicative of functionally active neurons), suggesting that they were active neuronal cells.

Conclusion: We showed for the first time that neoglucosylated collagen matrices may drive F11 cells and hMSC from proliferation to differentiation into neurons without the use of chemical differentiating agents.

\section{References}

[1] L. Russo, A. Gautieri, M. Raspanti, F. Taraballi, F. Nicotra, S. Vesentini, L. Cipolla. Carbohydrate functionalized collagen matrices: design and characterization of a novel neoglycosylated biomaterial. Carbohydr. Res. (2014);389:12-7

[2] L Russo, A Sgambato, M Lecchi, V Pastori, M Raspanti, A Natalello, SM. Doglia, F Nicotra, L Cipolla. Neoglucosylated collagen matrices drive neuronal cells to differentiate. ACS Chem Neurosci. (2014); 5:261-5

[3] A. Sgambato, L. Russo, M. Montesi, S. Panseri, M. Marcacci, E. Caravà, M. Raspanti, L. Cipolla* Different sialoside epitopes on collagen film surfaces direct mesenchymal stem cell fate. ACS Appl. Mater. Interfaces (2015);10.1021/ acsami. 5 b08270

\section{Oxidative stress and altered mitochondrial dynamics in neurodegenerative processes}

\author{
M. Formenti ${ }^{1}$, F. Aprea ${ }^{1}$, G. Sala ${ }^{2}$, C. Zoia ${ }^{2}$, \\ C. Riva ${ }^{2}$, D. Marinig ${ }^{2}$, F. Martorana ${ }^{1}$, B. Sala ${ }^{1}$, \\ E. Buglione ${ }^{1}$, A. Kolodkin ${ }^{3}$, L. Savarese ${ }^{4}$, \\ R. Balling ${ }^{3}$, M. Papa ${ }^{4}$, L. Alberghina ${ }^{5}$, C. Ferrarese ${ }^{6}$, \\ AM. Colangelo ${ }^{1}$ \\ ${ }^{1}$ Dept. of Biotechnology and Biosciences, \\ University of Milano-Bicocca (Milano) \\ ${ }^{2}$ School of Medicine and Surgery, University of \\ Milano-Bicocca (Milano) \\ ${ }^{3}$ LCSB, Luxembourg Centre for Systems \\ Biomedicine (Luxembourg-L) \\ ${ }^{4}$ Dept. of Mental and Physical Health and \\ Preventive Medicine, Second University of Naples \\ (Napoli)
}

${ }^{5}$ SYSBIO Centre of Systems Biology, University of Milano-Bicocca (Milano)

${ }^{6}$ Dept. of Neurology, San Gerardo Hospital (Monza)

Aims: Mitochondria are very dynamic organelles. Mitochondrial dynamics (alternation of fission/fusion cycles and their axonal transport) and biogenesis are crucial for neuronal homeostasis and synaptic function; moreover, alteration of the balance between fission and fusion can determine the fate of neuronal cells. Fission processes require the intervention of Fis 1 and the active form of Drp1, whereas fusion is primarily regulated by Opa1 and Mfn1/2. We here focused on correlation between oxidative stress and mitochondrial dynamics and biogenesis.

Materials and Methods: We compared the effect of hydrogen peroxide and Rotenone (a neurotoxin that inhibits the Complex-I of electrons transport chain), on primary cortical neurons or models of neurodegeneration based on neuroblastoma or PC12 cells. Protein levels and distribution (cytoplasm, mitochondria or nuclei) were assessed by western blots and immunofluorescence imaging.

Results: Exposure of neurons or neuroblastoma to Rotenone determined a dose-dependent (200-400$800 \mathrm{nM}$ ) decrease of P-Drp1 and a biphasic trend of Opa1 and Mfn2 in a time-dependent manner (1-3-624h). In PC12 cells, alterations of mitochondrial proteins in response to hydrogen peroxide was paralleled by differential distribution (cytoplasm/nuclei) of oxidative-stress sensors (DJ-1, Nrf2) and proteins regulating mitochondrial biogenesis ( $\mathrm{mtT}$ FAM and PGC1a), while determining a dose- and time-dependent increase of ROS and ATP depletion. Interestingly, human fibroblasts showed similar changes in response to rotenone but a different trend in response to Amyloid-beta oligomers.

Discussion/Conclusions: These studies suggest 1) the existence of a complex cross-talk underlying mitochondrial dynamics and biogenesis, 2) alteration of mitochondrial dynamic proteins as biomarkers of mitochondrial dysfunction in neurodegenerative processes. 


\section{Evaluation of in vitro antitumoral activity and neurotoxicity of a Hibiscus Sabdariffa ethyl acetate fraction against human multiple myeloma cells}

\author{
A. Malacrida ${ }^{1}$, R. Rigolio ${ }^{1}$, G. Nicolini ${ }^{1}$, \\ A. Cassetti ${ }^{2}$, G. Cavaletti ${ }^{1}$, M. Miloso ${ }^{1}$ \\ ${ }^{1}$ Department of Medicine and Surgery, University \\ of Milan-Bicocca (Milano) \\ ${ }^{2}$ Research Unit for Floriculture and Ornamental \\ Species, CRA (Sanremo)
}

Background: Hibiscus Sabdariffa is a plant of the Malvacee family commonly used in Asian and African folk medicine and for Karkadè preparation. In our laboratory, we previously demonstrated that Hibiscus Sabdariffa total extract (HSE) exerted against human multiple myeloma cells a reversible cytostatic effect and reduced cell motility/invasiveness. In order to identify the molecule/s involved in HSE effects, in this study we evaluated the antitumoral activity of a fraction of HSE obtained after ethyl acetate separation (HEF).

Material and methods: Human multiple myeloma cells RPMI8226 were treated with different HEF concentrations and cell viability was evaluated by MTT and Trypan blue vital count assays. Apoptosis cell death was evaluated by AnnexinV assay and Caspase 3 western blot. Cells migration/invasion was assessed using Boyden Chamber assay. Rat embryo dorsal root ganglia cultures were used to assess HEF neurotoxicity in vitro. HEF and HSE activity was compared.

Results: HEF was effective in reducing RPMI8226 cell viability in a dose and time dependent manner. HEF enhanced cell mortality, AnnexinV positive cells and Caspase 3 activation. Moreover, HEF was effective in reducing cells migration/invasion and it was not neurotoxic in vitro.

Conclusions: In this study we evaluated the in vitro effects of HEF against human multiple myeloma cells RPMI8226. Our results demonstrated that HEF treatment showed an increased effectiveness and Caspase3-dependent apoptosis, but not an increased neurotoxicity, compared to HSE. Considering evident anticancer properties of $\mathrm{HEF}$, it can provide the basis for a further fractionation in order to isolate molecules responsible for Hibiscus sabdariffa effects.

\section{Role of Glutathione in Mesenchymal Stem Cell mediated neuroprotection}

\author{
M. Monfrini, M. Ravasi, D. Maggioni, G. Tredici, \\ G. Cavaletti, A. Scuteri \\ Schoool of Medice and Surgery, University \\ Milano-Bicocca (Monza)
}

Mesenchymal Stem Cells (MSCs) have immunomodulatory properties and a supporting action on cellular survival; for these reasons MSCs have been proposed for the treatment of different diseases such as degenerative ones, both of CNS and PNS. It has been already demonstrated in vitro that rat MSCs support the survival and maturation of untreated sensory neurons from DRG Dorsal Root (Scuteri et al. 2006), but also that rMSCs are able to reduce Cisplatin effect on DRG neurons. Here we investigate the mediators of the neuroprotective effect of MSC. Dorsal Root Ganglia neuron primary cutures: DRG from 15-days-old embryonic Sprague-Dawley rats and MSCs from bone marrow of 10 weeks-old Sprague-Dawley rats. We set up direct and indirect co-cultures of DRG neurons and MSCs in order to test the MSC effect even after Paclitaxel exposure. We observed an increase of neuronal survival after co-cultures with MSC, even on Paclitaxel treated neurons. We focused our attention on soluble molecules which could be responsible of the positive effect of MSC; we identified a pivotal and specific role of glutathione, which was able to protect injured neurons from cellular death. Future studies should more deeply examine if MSCs, under which their peculiar properties, should be used for the protection of neurons exposed to other injuries, underlying other types of neurological disease of CNS (Alzheimer and Parkinson Disease, Multiple Sclerosis) or PNS (peripheral nerve damage).

\section{Reference}

[1] Scuteri A, Cassetti A, Tredici G. Adult mesenchymal stem cells rescue dorsal root ganglia neurons from dying. Brain Res. (2006) Oct 20;1116(1):75-81. Epub 2006 Sep 7 


\section{Targeting sirtuins may reduce oxidative stress and neurodegeneration in Parkinson's disease in vitro models}

\author{
I. Raimondi, S. Rodilossi, G. Forloni, D. Albani \\ Department of Neuroscience, IRCCS Mario Negri \\ Institute for Pharmacological Research (Milano)
}

Aims: Parkinson's disease (PD) is a prevalent neurodegenerative disorder that affects the motor system through a depletion of dopaminergic neurons. Several studies showed that the deacetylase sirtuins (SIRTs) are involved in neurodegeneration. In particular, SIRT2 promotes neurodegeneration, while SIRT1 is neuroprotective. Our study focuses on the role of SIRT1/SIRT2 modulation against oxidative stress in in vitro models.

Methods: SH-SY5Y neuroblastoma cells harboring the familial PD-linked DJ-1 protein mutation M26I, were treated with SIRT1 activators (resveratrol/SRT1720), SIRT2 inhibitor (AGK2) or a combination of the two, and then exposed to oxidative stress (H2O2 and 6-hydroxydopamine [6-OHDA]). Viability was assessed by MTT assay. Primary mesencephalic dopaminergic neurons were treated with AGK2 and assessed for neurites trophic status by tyrosine hydroxylase (TH) immunofluorescence and neurites length measurement.

Results: Oxidative stress reduced SH-SY5Y viability of around $40 \%$ and DJ-1 M26I clones were more vulnerable to the stress. In both cell types, treatments increased viability of about $20 \%$. Results dealing with a combined treatment indicated a survival enhancement. In primary cultures exposed to oxidative stress, AGK2 protected TH-positive neurons from fragmentation and retraction of neurites.

Discussion: SIRT1/SIRT2 modulation counteracted the effects of oxidative stress increasing cell viability also in DJ-1 clones, more sensitive to stress. In primary cultures, AGK2 didn't counteract neuronal reduction, but it exerted a trophic effect on dopaminergic population.

Conclusions: Our data support the relevance of SIRT1/SIRT2 modulation to ameliorate pathological features of PD linked to oxidative stress. The synergism of SIRTs-modulators is promising to create an effective neuroprotective strategy.

\section{References}

[1] Donmez G, Outeiro TF. SIRT1 and SIRT2: emerging targets in neurodegeneration. EMBO Mol Med. (2013) Mar;5(3):344-52
[2] Batelli S, Invernizzi RW, Negro A, Calcagno E, Rodilossi S, Forloni G, Albani D. The Parkinson's disease-related protein DJ-1 protects dopaminergic neurons in vivo and cultured cells from alpha-synuclein and 6-hydroxydopamine toxicity. Neurodegener Dis. (2015);15(1):13-23

[3] Albani D, Polito L, Batelli S, De Mauro S, Fracasso C, Martelli G, Colombo L, Manzoni C, Salmona M, Caccia S, Negro A, Forloni G. The SIRT1 activator resveratrol protects SK$\mathrm{N}-\mathrm{BE}$ cells from oxidative stress and against toxicity caused by alpha-synuclein or amyloid-beta (1-42) peptide. J Neurochem. (2009) Sep;110(5):1445-56

\section{Epigallocatechin-3-gallate and related compounds contrast ataxin-3 amyloid aggregation and relieve toxicity in a caenorhabditis elegans animal model}

M. E. Regonesi ${ }^{1}$, C. Visentin ${ }^{1}$, M. Bonanomi ${ }^{1}$, J. Modica ${ }^{1}$, A. Natalello ${ }^{1}$, C. Airoldi ${ }^{1}$, L. Amigoni ${ }^{1}$, SM. Doglia ${ }^{2}$, P. Tortora ${ }^{1}$

${ }^{1}$ Dept. of Biotechnologies and Biosciences, University of Milano-Bicocca (Milano)

${ }^{2}$ Dept. of Physics "Giuseppe Occhialini",

University of Milano-Bicocca (Milano)

Objectives: Ataxin-3 (ATX3) is a protein that triggers a neurodegenerative, amyloid-related disorder when its polyglutamine stretch exceeds a critical length [1]. The present investigation is aimed at developing/characterizing compounds capable of contrasting ATX3 fibrillation and toxicity.

Materials: A transgenic Caenorhabditis elegans strain was developed as reported [2].

Methods: Fourier Transform Infrared (FTIR) spectroscopy and NMR were performed as reported [2] and [3], respectively.

Results: We first evaluated the effects of epigallocatechin-3-gallate (EGCG) on ATX3 in vitro. By FTIR spectroscopy we observed that it interfered with the early steps of aggregation, accelerating the misfolding of the N-terminal Josephin Domain (JD), and leading to the formation of off-pathway aggregates. NMR analyses showed that EGCG is capable of binding to the JD, which is responsible for the onset of AT3 fibrillation [3]. These findings suggest that EGCG redirects fibrillation towards non-toxic aggregates, which was confirmed by its capability of significantly increasing the motility of a diseased C. elegans strain [2]. Other related polyphenol compounds exerted qualitatively similar effects on aggregation pathway and toxicity, although somewhat less pronounced.

Discussion: We have demonstrated the ability of different polyphenols to redirect ATX3 amyloid 
aggregation and mitigate the ensuing toxicity. In particular, the gallic acid moiety of EGCG offers the advantage of being substantially stable and also lends itself to be easily conjugated to nanovectors for the targeted delivery to the nervous system.

Conclusion: Our present investigations are focused on the development of nanovectors capable of targeting gallic acid and possibly other polyphenols to the brain.

\section{References}

[1] Li X, Liu H, Fischhaber PL, Tang TS. Toward therapeutic targets for SCA3: Insight into the role of Machado-Joseph disease protein ataxin-3 in misfolded proteins clearance. Prog Neurobiol (2015);132:34-58

[2] Bonanomi M, Natalello A, Visentin C, Pastori V, Penco A, Cornelli G, Colombo G, Malabarba MG, Doglia SM, Relini A, Regonesi ME, Tortora P. Epigallocatechin-3-gallate and tetracycline differently affect ataxin-3 fibrillogenesis and reduce toxicity in spinocerebellar ataxia type 3 model. Hum Mol Genet (2014);23:6542-6552

[3] Bonanomi M, Visentin C, Natalello A, Spinelli M, Vanoni M, Airoldi C, Regonesi ME, Tortora P. How Epigallocatechin-3gallate and Tetracycline Interact with the Josephin Domain of Ataxin-3 and Alter Its Aggregation Mode. Chemistry (2015);21:18383-18393

\section{Automatic Calibration of Cerebellar Stellate Neuronal Models using Swarm Intelligence}

\author{
M. F. Rizza, M. Nobile \\ Dept. Informatics, Systems and Communications, \\ University of Milan-Bicocca (Milano)
}

Aim: A new automatic methodology, which exploits the swarm intelligence technique known as Particle Swarm Optimization (PSO), was applied to estimate the ionic conductances of the cerebellar stellate cell (SC) model. SCs are involved, with the rest of network, in the Alzheimer's disease.

Methods: The SC model was implemented in Python-NEURON (Python 2.7; NEURON 7.3) with a realistic morphology and 15 ionic channels. The PSO was used to automatically determine the optimal solutions, using the time-series of neuron's activity as target. To reduce the computational costs, the fitness evaluations were accelerated with a computer cluster. We tested the methodology on a dual processor equipped with 12-cores, using the fixed time step of $0.025 \mathrm{~ms}$ and simulations of $2000 \mathrm{~ms}$. With this distributed architecture, a parameter estimation was performed in about 100 minutes, using 20 particles and 100 iterations.
Results: We obtained models, with 40 parameters estimated with the PSO, capable of reproducing the most important behaviors of SCs, such as spontaneous activity, fast responses to positive current injections, the activity generated by the interaction of coupled SC trough gap-junctions and their interaction in a large network.

Discussion: Detailed computational models of neurons are valuable means to investigate these complex cells. However, the most advanced models based on the Hodgkin-Huxley theory, require the fine-tuning of a large number of parameters to obtain the correct physiological behaviors. This is generally performed with a manual time-consuming trial and error process. In this work we investigated the alternative approach, based on the PSO, with promising results.

\section{Rotenone-induced down-regulation of HSPA8/hsc70 protein increases alpha- synuclein in vitro: possible implications for Parkinson's disease}

G. Sala ${ }^{1}$, D. Marinig ${ }^{2}$, C. Riva ${ }^{1}$, A. Arosio ${ }^{1}$, L. Brighina ${ }^{3}$, M. Formenti ${ }^{4}$, L. Alberghina, A. Colangelo ${ }^{5}$, C. Ferrarese ${ }^{1,3}$

${ }^{1}$ School of Medicine and Surgery, University of Milano-Bicocca (Monza)

${ }^{2}$ School of Medicine and Surgery, PhD program in Neuroscience, University of Milano-Bicocca (Monza)

${ }^{3}$ Dept. of Neurology, San Gerardo Hospital

(Monza)

${ }^{4}$ Dept. of Biotechnology and Biosciences, University of Milano-Bicocca (Milano)

${ }^{5}$ Dept. of Biotechnology and Biosciences, SYSBIO Centre of Systems Biology, University of MilanoBicocca (Milano)

Aims: HSPA8/hsc70 (70-kDa heat shock cognate) chaperone protein is involved in at least two critical processes whose activity is essential in preventing Parkinson's disease (PD) pathology. Actually, hsc70 protein acts as the main carrier of chaperone-mediated autophagy (CMA), a selective catabolic pathway for alpha-synuclein, and prevents/reduces alphasynuclein aggregation. Considering that the mitochondrial complex I inhibitor rotenone, used to generate PD animal models, induces alpha-synuclein aggregation, this study was designed to verify whether rotenone exposure leads to hsc 70 alteration 
possibly contributing to alpha-synuclein aggregation.

Materials and methods: Human SH-SY5Y neuroblastoma cells and primary mouse cortical neurons were treated with rotenone and hsc70 mRNA and protein expression was assessed by real-time pcr and Western blot, respectively. The effect of rotenone on hsc70 was compared with that exerted by hydrogen peroxide, a generic oxidative stress donor with no inhibitory activity on mitochondrial complex I. Furthermore, the influence of hsc70 gene knockdown on neurotoxicity was assessed through hsc70 gene silencing by small interfering RNA.

Results: Rotenone, but not hydrogen peroxide, induced a significant reduction of hsc70 mRNA and protein expression. Hsc70 gene silencing up-regulated alpha-synuclein mRNA and protein levels without affecting cell viability and without altering rotenone- and hydrogen peroxide-induced cytotoxicity.

Discussion: These findings demonstrate that HSPA $8 /$ hsc 70 chaperone protein represents a new intracellular target of rotenone toxicity contributing to alpha-synuclein accumulation.

Conclusions: Although the molecular mechanisms responsible for rotenone-induced hsc70 reduction need to be clarified, these findings suggest that hsc70 up-regulation may represent a promising therapeutic strategy for PD and other synucleinopaties.

\section{The one-carbon metabolism in anorexia nervosa of the restrictive type}

L. Tremolizzo, E. Conti, F. Corbetta, M. Bomba, F. Neri, C. Ferrarese, R. Nacinovich School of Medicine and NeuroMI, UNIMIB (Monza)

Objectives: Epigenetic dysfunctions are potential culprits in complex disorders such as anorexia nervosa of the restrictive type (AN). DNA methylation is the best-studied epimutation, able to induce profound changes in gene expression without altering the underlying base sequence. Aim of this work consisted in approaching various targets within the onecarbon metabolism in AN, especially when considering that the methylation potential is granted by the dietary intake of the essential amino acid methionine.

Materials and Methods: 32 AN female adolescents were recruited the Department of Child and Adolescent Neuropsychiatry of the San Gerardo
Hospital of Monza (Italy), together with 13 agecomparable drug-free control (CTRL) females. The following parameters were assessed: whole-blood global DNA methylation, serum vitamin B12 and B9, homocysteine (Hcy), beta-amyloid 40, leptin and steroid hormones. The ApoE genotype was obtained as well.

Results: Whole-blood global DNA methylation was significantly reduced and related to serum steroid hormone levels, while leptin was markedly downregulated. About a third of patients displayed increased serum Hcy and increased vitamin B12 levels correlating with disease severity. Beta-amyloid 40 was unchanged albeit correlating with Hcy levels (the ApoE genotype did not modify this result).

Conclusions: Our data suggest an impairment of the one-carbon metabolism at different levels and more work is needed for elucidating this matter. In particular, future work will consider also the activity of DNA methylating and demethylating enzymes in AN.

\section{References}

[1] Tremolizzo L, Conti E, Bomba M, Uccellini O, Rossi MS, Marfone M, Corbetta F, Santarone ME, Raggi ME, Neri F, Ferrarese C, Nacinovich R. Decreased whole-blood global DNA methylation is related to serum hormones in anorexia nervosa adolescents. World J Biol Psychiatry (2014);15:32733

[2] Corbetta F, Tremolizzo L, Conti E, Ferrarese C, Neri F, Bomba M, Nacinovich R. Paradoxical increase of plasma vitamin $\mathrm{B} 12$ and folates with disease severity in anorexia nervosa. Int J Eat Disord. (2015); 48:317-22

[3] Conti E, Nacinovich R, Bomba M, Raggi ME, Neri F, Ferrarese C, Tremolizzo L. Beta-amyloid plasma levels in adolescents with anorexia nervosa of the restrictive type. Neuropsychobiology (2015);71:154-7

\section{NEUROIMAGING}

\section{How many deficits in the same dyslexic brains? Behavioural and fMRI evidence}

L. Danelli ${ }^{1}$, M. Berlingeri ${ }^{2}$, M. Lucchese ${ }^{3}$,

A. Borghese ${ }^{3}$, M. Sberna ${ }^{4}$, G. Bottini ${ }^{5}$, E. Paulesu ${ }^{1}$

${ }^{1}$ Department of Psychology, University of Milano-

Bicocca (Milano)

${ }^{2}$ DISTUM, Department of Humanistic Studies,

University of Urbino Carlo Bo (Urbino)

${ }^{3}$ AIS-Lab and Department of Computer Science,

University of Milan (Milano)

${ }^{4}$ Neuroradiology Deparment, Niguarda Ca'

Granda Hospital (Milano)

${ }^{5}$ Cognitive Neuropsychology Center, Niguarda $\mathrm{Ca}$ '

Granda Hospital (Milano) 
Introduction: Dyslexia can have different manifestations, a fact that has motivated different theories (e.g. phonological, magnocellular, cerebellar) on its underlying brain bases. The relative weight of such comorbidities has never been evaluated with both behavioural and fMRI measures. To assess this issue, we combined behavioural and fMRI data in 20 adult well-compensated dyslexics and assessed multiple systems called into play in the dyslexia literature. Before and during fMRI scanning, reading abilities, auditory phonological, visual/magnocellular, and motor/cerebellar skills were assessed.

Results: Dyslexics were significantly slower in reading and in phonological manipulation tasks. Deficits in other domains were only very occasional. fMRI showed a reliable hypoactivation for pseudoword reading alone, in the left occipito-temporal cortex [1]. This finding was not accompanied by any other difference in any other task, nor as hypoactivations, nor as hyperactivations. The left occipito-temporal cortex is normally a region of functional intersection between visuo-magnocellular, or auditory phonological and the reading systems [2]. In dyslexics, the same area responded only to the auditory phonological task, on its most lateral part, or to the visuo-magnocellular task in its most posterior part.

Discussion: The present study demonstrates that in well-compensated adult dyslexics comorbidity is minimal. Our study also shows the power of the task of reading in bringing about significant reductions of brain activation in dyslexia. We suggest that dyslexics lack the maturation of a specialized orthographic decoding module in the left occipito-temporal cortex possibly because of a coarser connectivity between multiple domains that normally interact by anatomical convergence in that region.

\section{References}

[1] Paulesu E, Démonet JF, Fazio F, McCrory E, Chanoine V, Brunswick N, Cappa SF, Cossu G, Habib M, Frith CD, Frith U. Dyslexia: cultural diversity and biological unity. Science (2001);291(5511):2165-2167

[2] Danelli L, Berlingeri M, Bottini G, Ferri F, Vacchi L, Sberna M, Paulesu E. Neural intersections of the phonological, visual magnocellular and motor/cerebellar systems in normal readers: implications for imaging studies on dyslexia. Hum Brain Mapp (2013);34(10):2669-2687

\section{Functional effects of hand disuse in patients with trapeziometacarpal joint osteoarthritis}

\author{
M. Gandola ${ }^{1}$, L. Zapparoli' ${ }^{2}$, G. Saetta ${ }^{3}$, E. Rolandi², \\ P. Invernizzi ${ }^{2}$, A. De Santis ${ }^{4}$, G. Banfi ${ }^{5}$, M. Bruno ${ }^{4}$, \\ E. Paulesu ${ }^{2}$ \\ ${ }^{1}$ Department of Brain and Behavioral Sciences, \\ University of Pavia (Pavia) \\ ${ }^{2}$ Psychology Department, University of Milano- \\ Bicocca (Milano) \\ ${ }^{3}$ Neuropsychology Unit, Department of Neurology, \\ University Hospital Zurich (Zurich-CH) \\ ${ }^{4}$ IRCCS Istituto Ortopedico Galeazzi (Milano) \\ ${ }^{5}$ fMRI Unit, IRCCS Galeazzi \& University Vita e \\ Salute San Raffaele (Milano)
}

The human trapeziometacarpal joint has a crucial evolutionary importance as it permits the rotation/ opposition of the thumb to the other fingers [1]. There are clinical conditions, such as rhizarthrosis, in which this motor ability becomes difficult or impossible due to intolerable pain [2]. While behavioural consequences of rhizarthrosis are widely studied, the consequences of this disease on the cortical representation of hand movement are unknown. Nineteen patients with a diagnosis of rhizarthrosis and nineteen healthy subjects participated in the study. All participants performed specific tests to evaluate their motor (ME) and motor imagery (MI) abilities, pain intensity and the impact of rizoarthrosis on function outcome. The fMRI experiment involved a ME task and an explicit MI task of the same movement. Patients with rhizarthrosis were significantly slower than controls and lost the temporal congruence between ME and MI. The fMRI study showed that patients have a reduced response in the left sensory-motor cortex during the movement of the affected hand and grater activations in the dorsolateral premotor cortex during MI. Our data show consistent activation of contralateral premotor regions during MI with the affected hand, probably linked to compensatory processes together with the loss of activation, during the movement of the same hand, of brain regions, such as M1, more linked to the genesis of the motor outflow. To conclude, our results show that an orthopaedic disease, that reduces the motoric repertoire of the subjects in absence of any neurological impairment, induces neurofunctional changes in the cortical representation of hand movement. 


\section{References}

[1] Napier JR. Hands. Princeton, New Jersey: Princeton University Press. 1993

[2] Eaton RG \& Glickel SZ. Trapeziometacarpal osteoarthritis. Staging as a rationale for treatment. Hand Clin. (1987);3(4):455-471

\section{A Step in Mind: Neural Signatures of Motor Imagery and Imitation of Gait in Healthy Elderly}

L. M. Sacheli ${ }^{1}$, C. De Santis ${ }^{1}$, L. Zapparoli ${ }^{2}$, M. Preti ${ }^{1}$, C. Pelosi², B. Bodini², N. Ursino², A. Zerbi ${ }^{2}$, G. Banfi ${ }^{2}$, E. Paulesu ${ }^{1}$

${ }^{1}$ Department of Psychology, University of MilanoBicocca (Milano)

${ }^{2}$ IRCCS Orthopaedic Institute Galeazzi (Milano)

Introduction: Gait disturbances have a strong impact on daily life, e.g. in elderly or after prolonged immobilization. Clinical evidence suggests that both motor imagery [1] and observation of gait [2] favour gait rehabilitation. Here we aimed to describe the differences/similarities between the functional anatomical foundations of both processes, and to relate them with the brain activity associated with lower limbs explicit movements.

Methods: During fMRI, healthy elderly participants had to imagine standing (St) or walking (W) while observing stationary (St) or moving ("virtual walking", W) videos from a first person perspective. In $50 \%$ of videos, an actor was shown from the back as if he/she preceded the participant walking along the path. In $50 \%$ of trials, participants also performed ankle dorsiflexion [3] while observing the movies.

Results: Results showed that the neural resources recruited during $\mathrm{W}$ (with or without the actor) comprise the network recruited for ankle dorsiflexion execution - including, bilaterally, the supplementary motor area (SMA), paracentral lobule and the cerebellum- and additionally recruited the right Rolandic operculum and pre-SMA. Interestingly, while motor imagery and imagery of imitation shared similar cortical and cerebellar activations, motor imagery additionally recruited a cluster compatible with the human mesencephalic locomotor region.

Conclusions: These results show that motor imagery during "virtual walking" engages both cortical and subcortical networks implicated in gait control. Importantly, subcortical neural activity was modulated by the presence of an actor, suggesting a differential modulatory effect of walk imitation over the brainstem centres responsible for "internally generated" gait initiation.

\section{References}

[1] Malouin, F. \& Richards C.L. Mental practice for relearning locomotor skills. Physical Therapy (2010);90(2):240-251

[2] Pelosin, E. et al. Action observation improves freezing of gait in patients with Parkinson's disease'. Neurorehabilitation and Neural Repair (2010);24(8):746-752

[3] Dobkin, B.H. et al. Ankle dorsiflexion as an fMRI paradigm to assay motor control for walking during rehabilitation. Neuroimage (2004);23(1):370-381

\section{Nicotine boosts activation of frontal brain areas subserving working memory in visuospatial attention orienting}

\author{
A. Zani ${ }^{1}$, V. Lorenzi ${ }^{1}$, A. M. Proverbio ${ }^{2}$ \\ ${ }^{1}$ Institute of Molecular Bioimaging and Physiology \\ of National Research Council (CNR IBFM) \\ (Segrate-MI) \\ ${ }^{2}$ Milan Center for Neuroscience (NEUROMI), \\ Department of Psychology, University of Milan- \\ Bicocca (Milano)
}

Aims: Previous research provided controversial evidence of the amelioration of visuospatial attention orienting induced by nicotine both in animals (e. g., mice) and humans. Aim of our study was to investigate whether this amelioration might occur, and which brain areas were affected, by comparing healthy smokers ( $\mathrm{Sm})$ with non-smokers (NSm) engaged in challenging cognitive-motor tasks.

Materials and Methods: A central-cueing (CC), a local-cueing (LC), a local-cueing associated with a backward count (LCcount), and a local-cueing 2-choice motor (LCmot) visuospatial task were administered. Behavioral responses (RTs) and EEG to targets were recorded from 128 channels. Source reconstructions on difference waves (DW) were carried out by means of swLORETA.

Results: RTs orienting cost in the cognitively challenging condition, as derived by the difference between LCcount and LC, was $40 \mathrm{~ms}$ significantly lower in Sm than in NSm. In agreement with behavioral findings, at anterior scalp areas the LCcountLC DW in the ERPs P3 latency range was significantly smaller for $\mathrm{Sm}$ than for NSm. swLORETA computed in this latency range for the two groups showed, among others sources, an activation of brain BA9 and BA46 for Sm, but not NSm. 
Discussion and Conclusions: The consistency of our localizations with those of hemodynamic studies on memory recall lends support to the view of an expansion of working memory capacity during visuospatial attention orienting in $\mathrm{Sm}$, which might be probably caused by nicotine assumption through smoking. Possibly, then, in not-hurtful forms as smoking, nicotine might reveal useful for treating mnemonic symptoms in Alzheimer dementia.

\section{NEUROPSYCOLOGY AND BEHAVIOUR}

\section{Affective audio analysis using objective features}

\author{
F. Gasparini, S. Corchs, A. Grossi \\ DISCO, University of Milano Bicocca (Milano)
}

Several studies exist in the literature that address the problem of emotion classification of visual stimuli but less effort has been devoted to emotion classification of audio stimuli. The most of these studies start from the analysis of physiological signals such as EEG data [1]. The aim of this work is to evaluate if it is possible to classify audio signals according to elicited emotions using only objective features. In our analysis we adopt the IADS (International Affective Digitized Sound) database [2], composed of 167 auditory stimuli. The database provides pleasure, arousal and dominance ratings for each audio stimulus, recorded from 100 subjects during psycho physical test. The database is formed by different type of audio: from environmental sounds to music, as well as from single sound to complex ones. We start considering the affective dimension of valence within the three categorical classes of low, medium and high pleasure. To investigate this classification task we consider 35 features both in time and frequency domain. With these features, we test three types of classifiers: Bayesian, K Nearest Neighbor and Classification and Regression Tree [3]. We apply a feature selection strategy in order to find the more significant features. Using these features and the Bayesian classifier we have reached an average accuracy of $45 \%$. A similar result is achieved using physiological signals [1]. Starting from our results we believe that dividing each audio files in frames and applying a windowing strategy to evaluate objective features, the final classification performance could significantly increase.

\section{References}

[1] Koelstra, Sander, Christian Mühl, Mohammad Soleymani, Jong-Seok Lee, Ashkan Yazdani, Touradj Ebrahimi, Thierry Pun, Anton Nijholt, and Ioannis Patras. "Deap: A database for emotion analysis; using physiological signals." Affective Computing, IEEE Transactions on 3, no. 1 (2012): 18-31

[2] Bradley, Margaret M., and Peter J. Lang. "The International Affective Digitized Sounds (IADS-2): Affective ratings of sounds and instruction manual." University of Florida, Gainesville, FL, Tech. Rep. B-3 (2007)

[3] Duda, Richard O., Peter E. Hart, and David G. Stork. Pattern classification. John Wiley \& Sons (2012)

\section{Tool use and concept representation in the brain: a study on three neurodegenerative patients}

\author{
I. Mauri ${ }^{1}$, V. Isella ${ }^{2}$, C. Mapelli ${ }^{2}$, C. Luzzatti ${ }^{1}$ \\ ${ }^{1}$ Psychology, University of Milan-Bicocca (Milano) \\ ${ }^{2}$ Neurology Unit, School of Medicine (Monza)
}

Objectives: Apraxic patients with left-hemisphere lesions are impaired in semantic tasks tapping knowledge of objects manipulation, but the specific locus of actions representations is still debated: the same brain regions involved in action production, according to the sensory-motor theory, or the left posterior middle/superior temporal gyrus (MTG/ STG), according to a more recent hypothesis. To address this issue, we investigated brain FDG-PET correlates of action knowledge in three neurodegenerative cases with left-hemisphere damage.

Materials and methods: We included one patient with Cortico-basal Syndrome (CBS), limb apraxia and parietal-occipital hypometabolism, one patient with Alzheimer's Disease (AD), mild apraxia and aphasia and extensive posterior temporal hypometabolism including MTG/STG, and one patient with Semantic Dementia (SD) showing anterior temporal hypometabolism. Their knowledge of actions vs context of use was assessed with a visual object-toobject association task and a verbal categorical decision task.

Results: The CBS case showed no impairment of action knowledge, the AD patient had a worse performance in the gesture condition, with a strong dissociation in the verbal task, and the SD case was equally impaired in gesture and context in the verbal task but had a normal performance with gestures in the visual task.

Discussion and conclusions: Contrary to the sensory-motor prediction, in the CBS patient parietal damage causing apraxia dissociated from loss of 
action knowledge. In the SD case, general semantic impairment affected action representations, sparing access to affordances. Finally, the AD patient's performance supports a specific role for the left posterior MTG/STG in storing/processing action semantics.

\section{References}

[1] Binkowski F., Buxbaum L. J. Two action system in the human brain. Brain and Language (2012);127:222-229

[2] Buxbaum L. J. et al. Critical brain regions for tool-related and imitative actions: a componential analysis. Brain (2014);137(7):1971-1985

[3] Ishibashi R. et al. Different roles of lateral anterior temporal lobe and inferior parietal lobule in coding function and manipulation tool knowledge: Evidence from an rTMS study. Neuropsychologia (2011);49:1128-1135

\section{Neuro-cognitive disorders following hip fracture: a retrospective analysis on patients who had surgery and post- surgical rehabilitation}

\author{
R. Meroni ${ }^{1}$, C. Perin ${ }^{2}$, R. Bertoja ${ }^{1}$, C. Cornaggia ${ }^{2}$, \\ E. Gualandris ${ }^{2}$, C. Cerri ${ }^{2}$ \\ ${ }^{1}$ Istituti Clinici Zucchi, GSD (Carate Brianza-MB) \\ ${ }^{2}$ School of Medicine and Surgery, University of \\ Milan Bicocca (Monza)
}

Aims: Cognitive deficits are commonly observed in elders who underwent surgery. The aim of this observational retrospective study was to compare the incidence of neuro-cognitive disorder (NCD DSM $\mathrm{V}$ ) and its effects on the rehabilitation outcome after surgery in two different scenarios: emergency surgery for hip fracture (HF) and planned surgery for hip replacement (HR).

Methods: Retrospective analysis. Inclusion criteria: hospitalized after HF or HR surgery, age at surgery $>65$ years. Exclusion criteria: polytrauma, stroke, exitus, paraplegia, extra-pyramidal syndromes, amputation and cancer.

Statistics: Exploratory analysis, cross-tabulation, chi-square test and risk analysis were performed on categorical variables. t-test or MW test were performed on continuous variables. Variables associated with cognitive decay have been analyzed using a binary logistic regression.

Results: 277 patients. 166 subjects underwent HF surgery, 111 subjects underwent HR surgery. HF patients were older ( $81 \mathrm{y}$ vs $75 \mathrm{y})$, their prevalence of NCD was higher $(35,5 \%$ vs $5,4 \%)$. Sight and hear- ing were more compromised in $\mathrm{HF}(44,9 \%$ vs $18,4 \%$ and $30,6 \%$ vs $22,9 \%$ ). NCD was associated with poorer Barthel Index (BI) scores at discharge. The rehabilitative prognosis was poor for HF patients with a lower BI and a longer hospitalization in the surgery ward.

Discussion: Prevalence of NCD was higher in HF patients. A relationship between the traumatic event and NCD is suggested. Subjects with NCD had poorer functional outcomes at discharge.

Conclusions: NCD is often associated with traumatic events and that its contribution to disability and dependence could be high.

\section{Influence of endarterectomy in asymptomatic carotid stenosis on cognitive function: longitudinal evaluation of neuropsychological changes}

R. Regazzoni ${ }^{1}$, G. Giussani ${ }^{1}$, E. Leo $^{2}$, E. Botto ${ }^{1}$, S. Ferrari $^{2}$, A. Molinari ${ }^{2}$, G. Rossi ${ }^{2}$, C. Scaccabarozzi ${ }^{1}$, A. Terruzzi ${ }^{1}$, A. Terzi ${ }^{2}$, G. Lorenzi ${ }^{2}$, A . Salmaggi ${ }^{1}$ ${ }^{1}$ Neuroscience Department, Neurology-Stroke Unit, ASST of Lecco (Lecco)

${ }^{2}$ Cardiovascular Department, Division Vascular Surgery, ASST of Lecco (Lecco)

Background: Our purpose was to study the effects of endarterectomy on cognitive performance in patients with asymptomatic carotid stenosis, investigating short and long-term impact of revascularization procedure on several cognitive functions by neuropsychological evaluation before surgery and after 12 months.

Methods: A total of 22 patients, 17 males and 5 females, mean age 72, 25 years, with asymptomatic carotid artery stenosis greater than $70 \%$ (NASCET criteria) and with MMSE score $>23.8$ undergoing carotid revascularization by CEA were enrolled. They were evaluated with neurological and neuropsychological testing at baseline (pre-operative). A subgroup of patients were also studied with baseline and post-operative diffusion-weighted MR imaging. The neurological clinical examination included also estimation of cerebrovascular reactivity with BHI-TCD. The neuropsychological battery was designed to evaluate function across the major cognitive domains (MMSE, language, memory, attention, executive function, reasoning, constructional abilities), 
mood, motor speed. 15 patients (10 males, 5 females with a mean age of 72,53 years) completed neuropsychological testing prior to and 12 months following the revascularization procedure (7 patient missed the follow-up consultation. A comparison of test results of the pre-procedural neuropsychological assessment demonstrated no significant differences between included and excluded patients).

Results: After CEA no significant changes at 1 year in the investigated parameters occurred although a trend to delayed improvement in verbal memory was seen in younger patients.

Conclusion: CEA in asymptomatic patients in our cohort was followed by stable cognitive status at 1 year. Further study will investigate relationships between cognitive changes and brain perfusion assessed by DWI-MRI and BHI-TCD.

\section{References}

[1] De Rango V., Leys D. et al. The role of carotid artery stenting and carotid endarterectomy in cognitive performance. A systematic review. Stroke (2008);39:3116-3127

[2] Stern Y. What is cognitive reserve? Theory and research application of the reserve concept. J Intern Neuropsychol Society (2002);8(3):448-460

[3] Wasser K., Hildebrandt H., Groschel S., Stojanovic T., Schmidt H., Groschel K., Pilgram-Pastor S., Knauth M., Kastrup A. Age-dependent effects of carotid endarterectomy or stenting on cognitive performance. J. Neurol. (2012);259:2309-2318

\section{Finding the dyadic motor plan: cognitive bases of motor interactions}

L. M. Sacheli, E. Arcangeli, E. Paulesu

Department of Psychology, University of Milano-

Bicocca (Milano)

The ability to interact with conspecifics is crucial in life, yet there is no consensus on the underlying cognitive mechanisms beyond those associated with imitative behaviors. This study aims to define a coherent model of motor interactions by testing the hypothesis that they are based on the ability to integrate one's own and a partner's action within a unitary, dual-person (dyadic), motor plan that incorporates a shared goal. With a novel "minimally-joint" paradigm, we analysed participants' performance in a grasping task, which requires them performing movements that could be either congruent or incongruent with a virtual partner's ones. In two different experimental conditions, participants performed the action in turn taking with the virtual partner, while either having (Joint Action condition, JA) or not (Independent condition, IND) the overarching goal of playing a pre-learned melody together with him. Namely, while in the IND condition the participant' and partner's movements are unrelated, in the JA one they are inherently linked by the overarching shared goal, and participants can thus predict the partner's action while planning their own motor response. We show that visuo-motor interference (as indexed by the comparison between performance in incongruent and congruent movements) was modulated by the presence of the overarching goal, suggesting that motor simulation of an observed partner's action is influenced by the overarching shared goal characterizing interacting contexts.

\section{Declarative long-term memory and the temporal lobe: insights from refractory epilepsy}

\author{
G. Salvato ${ }^{1}$, P. Scarpa $^{1}$, S. Francione ${ }^{2}$, E. Scarano ${ }^{1}$, \\ R. $\mathrm{Mai}^{2}$, L. Tassi ${ }^{2}$, G. Lo Russo ${ }^{2}$, G. Bottini ${ }^{1}$ \\ ${ }^{1}$ Cognitive Neuropsychology Centre, ASST Grande \\ Ospedale Metropolitano, Niguarda Hospital \\ (Milano) \\ ${ }^{2}$ Epilepsy Surgery Centre, ASST Grande Ospedale \\ Metropolitano, Niguarda Hospital (Milano)
}

Background: It is largely recognized that the mesial temporal lobe and its substructure support declarative long-term memory (LTM). So far, different accounts have been suggested, and the organization of declarative verbal LTM in the brain is still a matter of debate.

Aim: In the current study we aimed at investigating the features of declarative verbal LTM in patients suffering from drug-resistant TLE, and the modulation of memory performance of these patients induced by the surgical treatment of TLE.

Methods: We retrospectively selected before and after surgery 151 right-handed TLE patients with and without HS, with a homogeneous (seizure-free) clinical outcome. We analysed verbal memory performance within a normalized scores context, by means of associate learning and episodic LTM tasks. Patients were tested at pre-surgical baseline, 6 months, 2 and 5 years after surgery, using parallel versions of the neuropsychological tests.

Results: Our main finding revealed a key involvement of the left temporal lobe and, in particular of 
the left hippocampus in verbal episodic LTM rather than verbal associative learning. We also confirmed that the duration of epilepsy predicted the memory outcome. Remarkably, when individual memory performance was taken into account, data showed that females affected by left temporal lobe epilepsy for longer were more at risk of presenting a clinically pathologic episodic LTM at 5 years after surgery.

Conclusion: Taken together, these findings shed new lights on verbal declarative memory in the mesial temporal lobe, and on the behavioural signature of the functional reorganization after the surgical treatment of temporal lobe epilepsy.

\section{Task-driven influence of visual working memory on binocular rivalry}

L. Scocchia ${ }^{1}$, M. Valsecchi ${ }^{2}$, N. Stucchi ${ }^{3}$

${ }^{1}$ Department of Psychology, University of Milano-

Bicocca (Milano)

${ }^{2}$ Department of Psychology, Giessen University

(Giessen-D)

${ }^{3}$ Department of Psychology, Milan Center for

Neuroscience, University of Milano-Bicocca

(Milano)

One of the most prominent functions of visual working memory (VWM) is to actively maintain information for forthcoming goal-directed behaviour. Accordingly, the content of VWM may influence the selection of visual input for perceptual processing and be employed to guide perception in conditions were the incoming information is ambiguous to the point of generating bistability. This study investigates whether binocular rivalry, which occurs when incompatible monocular images are displayed at the same retinal location in the two eyes, can be influenced by the contents of VWM. In two experiments we showed that this is the case: actively storing an object that largely overlaps with either of the rival images in terms of low-level visual features influences rivalry perception. If an ambiguous percept that matches the VWM content is favoured at dominance onset, the sustained effect of VWM maintenance is to suppress the matching percept. This arguably occurs because perceiving an image that is visually similar to the VWM sample during rivalry would be detrimental to the VWM task, as the currently perceived image would interfere with the VWM sample for subsequent recall. The sustained effect of suppression of a matching percept is specific for the VWM task and cannot be ascribed to the effects of attention or visual pre-exposure. Our results support the idea of a non-automatic, goal directed influence of VWM on perception.

\section{References}

[1] Sreenivasan, K. K., Curtis, C. E., \& D’Esposito, M. Revisiting the role of persistent neural activity during working memory. Trends in cognitive sciences (2014);18(2):82-89

[2] Harrison, S. A., \& Tong, F. Decoding reveals the contents of visual working memory in early visual areas. Nature (2009);458(7238):632-635

\section{Mental motor representations across the adult life-span: behavioural and fMRI evidence in explicit and implicit motor imagery tasks}

\author{
L. Zapparoli ${ }^{1}$, A. Zerbi ${ }^{2}$, G. Banfi ${ }^{3}$, E. Paulesu ${ }^{4}$ \\ ${ }^{1}$ IRCCS Galeazzi, University of Milano-Bicocca \\ (Milano) \\ ${ }^{2}$ fMRI Unit, IRCCS Galeazzi (Milano) \\ ${ }^{3}$ Scientific Direction, IRCCS Galeazzi (Milano) \\ ${ }^{4}$ Psychology Department, University of Milano- \\ Bicocca (Milano)
}

Motor imagery (MI) is a cognitive state in which movements are evoked mentally. There is behavioural evidence that MI declines with aging, but limited is the information available on the functional anatomical correlates of these changes. Here we present a behavioural/fMRI investigation of this issue based on the study of 60 elderly and 60 young subjects (mean age: 61 and 31 years) with explicit and implicit MI paradigms of different complexity. We found different behavioural and fMRI patterns, depending on the task: in the simple implicit MI task (hand-laterality task) older subjects had a normal behavioural performance in terms of accuracy and latency: this was mirrored by the over-activations in the occipital cortices, whose response was positively correlated with the behaviour. The same was not true for the explicit MI of finger oppositions, where we found a "partially successful" compensatory neural pattern: occipital hyperactivations in elderly subjects were mirrored by with the loss of temporal congruency between real and imagined movements. This finding suggests an initial decline in MI abilities and suggests that occipital hyperactivations are compensatory attempts. The MI decline becomes 
more evident with a more complex implicit MI task (grip-selection task): here we found a behavioural decline accompanied by hypoactivation of the posterior parietal lobule, a key region for mental representations of reaching movements. These results demonstrate that aging in MI is associated with modified brain responses ranging from successful compensation to failed compensation, depending on the complexity and the kinematic requirements of the MI task.

\section{References}

[1] Zapparoli L, Invernizzi P, Gandola M, Verardi M, Berlingeri M, Sberna M, De Santis A, Zerbi A, Banfi G, Bottini G,
Paulesu E. Mental images across the adult lifespan: a behavioural and fMRI investigation of motor execution and motor imagery. Exp Brain Res. (2013)Feb;224(4):519-40

[2] Zapparoli L, Invernizzi P, Gandola M, Berlingeri M, De Santis A, Zerbi A, Banfi G, Paulesu E. Like the back of the (right) hand? A new fMRI look on the hand laterality task. Exp Brain Res. (2014)Dec;232(12):3873-95

[3] Zapparoli L, Saetta G, De Santis C, Gandola M, Zerbi A, Banfi G, Paulesu E. When I am (almost) 64: The effect of normal ageing on implicit motor imagery in young elderlies. Behav Brain Res. (2016) Apr 15;303:137-51 


\section{Author Index}

\begin{tabular}{|c|c|c|c|}
\hline Abate, G., & S19, S37 & Bini, P., & $\mathrm{S} 17, \mathrm{~S} 27, \mathrm{~S} 29$ \\
\hline Abete, C., & S39 & Biscetti, L., & $\mathrm{S} 40$ \\
\hline Abutalebi, J., & S43 & Blin, O., & S11 \\
\hline Agosti, V., & S42 & Boda, E., & S33 \\
\hline Agostini, S., & S29 & Bodini, B., & S63 \\
\hline Agrillo, A., & $\mathrm{S} 17$ & Bomba, M., & S61 \\
\hline Agosta, F., & S9 & Bonanni, L., & S5 \\
\hline Airoldi, C., & S30, S59 & Bonanomi, M., & S59 \\
\hline Albani, D., & $\mathrm{S} 11, \mathrm{~S} 17, \mathrm{~S} 59$ & Bonaretti, L., & $\mathrm{S} 22$ \\
\hline Albargothy, N., & $\mathrm{S} 3$ & Bordet, R., & $\mathrm{S} 11$ \\
\hline Alberghina, L., & S57, S60 & Borella, E., & $\mathrm{S} 23$ \\
\hline Alberti, P., & S54 & Borghese, A., & S61 \\
\hline Aliprandi, A., & S33 & Bottini, G., & S6, S22, S25, S45, \\
\hline Amanzio, M., & S48 & & S61, S66 \\
\hline Ambrogio, T., & $\mathrm{S} 45$ & Botto, E., & S65 \\
\hline Amigoni, L., & S59 & Botto, L., & S34, S36 \\
\hline Andreoni, S., & S35, S39 & Braida, B., & S33 \\
\hline Appollonio, I., & S23, S33, S51 & Brighina, L., & S60 \\
\hline Aprea, F., & S57 & Bruno, M., & S62 \\
\hline Arabia, G., & $\mathrm{S} 19$ & Buffo, A., & $\mathrm{S} 33$ \\
\hline Arcangeli, E., & S66 & Bugiani, O., & $\mathrm{S} 1$ \\
\hline Arosio, A., & S33, S55, S60 & Buglione, E., & S57 \\
\hline Asti, A., & $\mathrm{S} 30$ & Bulbarelli, A., & $\mathrm{S} 34, \mathrm{~S} 36$ \\
\hline Ayakta, N., & S44 & Bussei, R., & $\mathrm{S} 30$ \\
\hline Baggi, F., & $\mathrm{S} 30$ & Cabinio, M., & S16, S29, S34 \\
\hline Baglio, F., & S16, S29, S34, S47 & Caffarra, P., & $\mathrm{S} 23, \mathrm{~S} 24$ \\
\hline Balducci, C., & $\mathrm{S} 4, \mathrm{~S} 13, \mathrm{~S} 36$ & Calabrese, E., & $\mathrm{S} 29, \mathrm{~S} 34$ \\
\hline Balestrino, M., & $\mathrm{S} 22$ & Calabresi, P., & S40 \\
\hline Ballarini, T., & $\mathrm{S} 18, \mathrm{~S} 43, \mathrm{~S} 44$ & Calbrese, E., & $\mathrm{S} 16$ \\
\hline Balling, R., & S57 & Caminiti, S., & $\mathrm{S} 18$ \\
\hline Banfi, G., & S62, S63, S67 & Campini, I., & $\mathrm{S} 26$ \\
\hline Bartrés-Faz, D., & $\mathrm{S} 11$ & Cappa, S., & S46 \\
\hline Baselice, F., & $\mathrm{S} 42$ & Cappa, S. F., & S5 \\
\hline Basilico, S., & S45 & Carare, R. O., & $\mathrm{S} 3$ \\
\hline Beeg, M., & S33 & Cardamone, R., & $\mathrm{S} 31$ \\
\hline Beghi, E., & $\mathrm{S} 23$ & Carlesimo, G. A., & S7 \\
\hline Beghi, M., & S51 & Carpinelli, A., & $\mathrm{S} 40$ \\
\hline Belloli, S., & $\mathrm{S} 40, \mathrm{~S} 41$ & Caruso, F., & S27 \\
\hline Beltramello, A., & $\mathrm{S} 11$ & Casey, D., & $\mathrm{S} 21$ \\
\hline Bentivegna, A., & S54 & Cashman, J., & $\mathrm{S} 27$ \\
\hline Berlingeri, M., & S6, S22, S25, S61 & Cassetti, A., & S58 \\
\hline Bernasconi, M., & $\mathrm{S} 25$ & Catricala', E., & S46 \\
\hline Bertoja, R., & S65 & Caulo, M., & $\mathrm{S} 11$ \\
\hline Bertoli, M., & S49 & Cavaletti, G., & S54, S55, S58 \\
\hline Bianchi, E., & $\mathrm{S} 23$ & Cecchetti, G., & $\mathrm{S} 25, \mathrm{~S} 31$ \\
\hline Biella, G., & S17 & Cerami, C., & $\mathrm{S} 18, \mathrm{~S} 41$ \\
\hline
\end{tabular}


Cereda, C.,

Cereda, D.,

Ceresa, C.,

Cerizza, M.,

Cerri, C. G.,

Cerri, C.,

Chen, T.,

Chiao, P.,

Chiarini, P.,

Chieffo, R.,

Chipi, E.,

Chiriaco, C.,

Ciaramelli, C.

Cilibrasi, C.,

Ciocca, G.,

Cipolla, L.,

Ciresa, S.,

Ciricugno, A.,

Clerici, M.,

Coco, S.,

Colangelo, A. M.,

Colangelo, A.,

Colasuonno, F.,

Colombo, L.,

Comi, G.,

Consonni, A.,

Consonni, M.,

Conti, E.,

Corbetta, F.,

Corchs, S. E.,

Corchs, S.,

Corna, G.,

Cornaggia, C.,

Costaggiu, D.,

Cova, I.,

Curinga, T.,

Da Re, F.,

Dalla Bella, E.,

Danelli, L.,

d'Arma, A.,

De Luigi, A.,

De Santis, A.,

De Santis, C.,

Della Sala, S.,

Di Francesco, J. C.,

Di Grigoli, G.,

Di Luca, M.,

DiFrancesco, J.,

Dodich, A.,

Doglia, S. M.,
$\mathrm{S} 17, \mathrm{~S} 27, \mathrm{~S} 29$
$\mathrm{~S} 39$

S55

S56

S45

S52, S65

$\mathrm{S} 12$

S12

S40

S14

S40

S19

S30

S54

S51

S37, S56

S45

S45

S16, S29, S34

S16, S34

S57

S60

S32

S30

S25, S31

$\mathrm{S} 30$

$\mathrm{S} 46$

S32, S33, S39, S4,

$\quad$ S61
S61
S51
S64
S53
S51, S65

S39

S26

S25

S32

S46

S61

S47

$\mathrm{S} 30$

S62

S22, S63

S7

S23

S40, S41

$\mathrm{S} 2$

S35

S41

S59
Donato, F., $\quad$ S22

D'Onofrio, G., $\quad$ S21

D'Onofrio, M., $\quad$ S46

Dore, G., $\quad$ S46

Dubois, B., S8

Elia, C. A., $\quad$ S16

Epelbaum, S., $\quad$ S8

Eusebi, P., $\quad$ S40

Faggian, S., $\quad$ S23

Falautano, M., $\quad$ S25

Fallanca, F., $\quad$ S18

Farina, E., $\quad$ S47

Farina, F., $\quad$ S34, S36

Farotti, L., $\quad$ S40

Farsad, M., $\quad$ S43

Federico, A., $\quad$ S26

Fernandez, I., $\quad$ S25

Ferrarese, C.,

Ferrari, C.,

Ferrari, S.,

Ferri, A.,

Fiedler, U.,

Filipello, F.,

S4, S23, S32, S33, S39, S53, S55, S57, S60, S61

S11

S65

S53

S11

S16

Floridi, P.,

$\mathrm{S} 40$

Forloni, G.,

Formenti, M.,

S4, S11, S13, S17, S36, S59

S57, S60

S32

Fracassi, A.,

Frackowiak, R., $\quad$ S8

Francione, S., $\quad$ S66

Franzin, A., $\quad$ S31

Frisoni, G. B., $\quad$ S11

Frisoni, G., $\quad$ S18

Fumagalli, G., $\quad$ S54

Fusco, F., $\quad \mathrm{S} 17$

Gabrielli, M., $\quad$ S33

Gagliardi, S., $\quad$ S27

Galluzzi, S., $\quad$ S11

Gamberini, G., $\quad$ S47

Gandin, V., $\quad$ S55

Gandola, M., $\quad$ S62

Gandolfi, M., $\quad$ S26

Gardini, S., $\quad$ S23, S24

Gardoni, F., S33

Garibotto, V., $\quad$ S43

Gasparini, F., $\quad$ S51, S64

Gavazzo, P., $\quad$ S56

Gelosa, G., $\quad$ S45

Gerardi, F., S55 


\begin{tabular}{|c|c|}
\hline Gessa, V., & S46 \\
\hline Gianelli, M., & $\mathrm{S} 20$ \\
\hline Gioda, M., & S52 \\
\hline Giovannoni, R., & S54 \\
\hline Giuliani, F., & $\mathrm{S} 21$ \\
\hline Giussani, C., & S54 \\
\hline Giussani, G., & S23, S65 \\
\hline Gobbi, M., & S33 \\
\hline Gorno-Tempini, M., & $\mathrm{S} 44$ \\
\hline Grana, D., & S32, S33, S55 \\
\hline Grande, G., & S26 \\
\hline Greco, A., & $\mathrm{S} 21$ \\
\hline Greco, L. C., & S41 \\
\hline Gregori, M., & S32, S39 \\
\hline Grieco, G. S., & $\mathrm{S} 17, \mathrm{~S} 29$ \\
\hline Grimaldi, R., & S27 \\
\hline Grossi, A., & S64 \\
\hline Gualandris, E., & S65 \\
\hline Guerini, F. R., & S29 \\
\hline Guizzardi, R., & S56 \\
\hline Hao, K., & S28 \\
\hline Heneka, M. T., & S3 \\
\hline Hernis, A., & S29 \\
\hline Ho, L., & S28 \\
\hline Hock, C., & $\mathrm{S} 12$ \\
\hline Hoeschele, J., & S55 \\
\hline Iaccarino, L., & $\mathrm{S} 41, \mathrm{~S} 44$ \\
\hline Iannaccone, S., & $\mathrm{S} 41$ \\
\hline Invernizzi, P., & S62 \\
\hline Isella, V., & S64 \\
\hline Iurato, L., & S27 \\
\hline Jacini, F., & $\mathrm{S} 42$ \\
\hline Jagust, W., & S44 \\
\hline James, O., & $\mathrm{S} 21$ \\
\hline Jelle Visser, P., & $\mathrm{S} 11$ \\
\hline Joshi, P., & S33 \\
\hline Jovicich, J., & S11 \\
\hline Knezovic, A., & S38 \\
\hline Kolodkin, A., & S57 \\
\hline La Rosa, F., & S34 \\
\hline Lanfranconi, F., & S53 \\
\hline Larosa, F., & $\mathrm{S} 16$ \\
\hline Lauria, G., & S46 \\
\hline Lazzati, G., & S47 \\
\hline Lecchi, M., & S56 \\
\hline Leo, E., & S65 \\
\hline Leocani, L., & $\mathrm{S} 14$ \\
\hline Lo Russo, G., & S66 \\
\hline Lonati, E., & $\mathrm{S} 34, \mathrm{~S} 36$ \\
\hline Longoni, M., & S35 \\
\hline Lorenzi, G., & S65 \\
\hline
\end{tabular}

Lorenzi, V., $\quad \mathrm{S} 63$

Losurdo, M., $\quad$ S16

Lozano, A. M., $\quad$ S15

Lubian, F., $\quad$ S43

Lucchelli, F., $\quad$ S47

Lucchese, M., $\quad$ S61

Lunetta, C., $\quad$ S53, S55

Luzzatti, C., S47, S64

MacGregor-Sharp, M., $\quad$ S3

Maggiani, A., $\quad$ S52, S53

Maggioni, D., $\quad$ S58

Maggioni, L., $\quad$ S33

Magnani, G., $\quad$ S25, S31, S41,

Mai, R., $\quad$ S66

Maier, A., $\quad$ S26

Malacrida, A., $\quad$ S58

Malpetti, M., $\quad$ S43

Mancini, S., $\quad$ S13, S36

Mancuso, R., $\quad$ S29

Mandas, A., $\quad$ S39

Mantegazza, R., $\quad$ S30

Mapelli, C., $\quad$ S64

Mapelli, D., $\quad$ S26

Marcello, E., $\quad$ S2, S33

Marchesi, N., $\quad$ S30

Marchetti, S., $\quad$ S16

Margiotta, N., $\quad$ S55

Mariani, C., $\quad$ S26

Marinig, D., $\quad$ S57, S60

Marizzoni, M., $\quad$ S11

Markova, A., $\quad$ S26

Marsili, G., $\quad$ S49

Martini, E., $\quad$ S47

Martorana, F., $\quad$ S57

Marventano, I., $\quad$ S16, S34

Marziano, M., $\quad$ S19, S37

Marzorati, M., $\quad$ S49

Masiello, V., $\quad$ S41

Masserini, M., $\quad$ S13, S32, S36,

S37, S39

Matteoli, M., S16, S33

Mauri, I., $\quad$ S64

Mazzeo, S., $\quad$ S25, S31

Mazzitelli, S., $\quad$ S33

Mazzola, P., $\quad$ S28

Mazzoni, V., $\quad$ S30

Meroni, R., S51, S52, S65

Michelini, G., $\quad$ S23, S24

Milani, C., S34, S36

Miller, B.,

S44 
Miloso, M., $\quad$ S5

Modica, J., $\quad$ S59

Molinari, A., $\quad$ S65

Monfrini, M., $\quad$ S58

Monterisi, C., S40, S41

Morciano, E., S23, S24

Morelli, M., $\quad$ S19

Moreno, S., $\quad$ S32

Moresco, R. M., $\quad$ S3

Moresco, R., S40, S41

Morris, A., $\quad$ S3

Murphy, K., $\quad$ S21

Murtaj, V.,

Musicco, M.,

Nacinovich, R.,

Naso, M. G.,

Natalello, A.,

Negroni, C.,

Nemni, R.,

Neri, F.,

Nestor, P. J.,

Nicoletti, G.,

Nicolini, G.,

Nicotra, F.,

Nitsch, R. M.,

Nobile, M.,

Nobili, F.,

Novellino, F.,

Occhipinti, C.,

O'Gorman, J.,

Onofrj, M.,

Osmanovic Barilar, J.,

Palermo, S.,

Palestini, P.,

Palmioli, A.,

Panina-Bordignon, P.,

Pannese, M.,

Papa, L.,

Papa, M.,

Parnetti, L.,

Pascale, A.,

Pasinetti, G.,

Passerini, G.,

Passoni, S.,

Pastori, V.,

Paulesu, E.,

Payoux, P.,

Pellei, M.,

Pelliccari, L.,

Pelosi, C.,

\section{S40, S41}

S1

S61

S27

S59

$\mathrm{S} 54$

S16, S29, S34, S47

S61

S5

S19

S55, S58

S30, S37

$\mathrm{S} 12$

$\mathrm{S} 60$

S11, S18

S19

S27

$\mathrm{S} 12$

S5

S38

S48

S34, S36

$\mathrm{S} 30$

S40

$\mathrm{S} 40$

S52

S57

S10, S11, S18, S40

$\mathrm{S} 30$

S28

$\mathrm{S} 25, \mathrm{~S} 31$

$\mathrm{S} 25$

S56

S9, S22, S61, S62,

S63, S66, S67

S11

S55

S52

S63
Pelucchi, S.,

Perani, D.,

Perin, C.,

Peroni, F.,

Petecchia, L.,

Piancone, F.,

Piazza, F.,

Picelli, A.,

Pilotto, A.,

Pinto, P.,

Piscitelli, D.,

Policicchio, S.,

Polita, M.,

Polonia, V.,

Pomati, S.,

Ponzoni, L.,

Porcelli, S.,

Pradelli, S.,

Presotto, L.,

Prete, C.,

Preti, M.,

Proverbio, A. M.,

Puntoni, M.,

Quattrone, A.,

Raccagni, I.,

Raimondi, I.,

Rainero, I.,

Randisi, M. G.,

Ranjeva, J. -P.,

Rasile, M.,

Ravasi, M.,

Re, F.,

Redaelli, S.,

Regazzoni, R.,

Regonesi, M. E.,

Restelli, A.,

Reutter, W.,

Ricca, I.,

Ricciardi, F.,

Richardson, J.,

Rigamonti, A.,

Rigolio, R.,

Ripamonti, E.,

Riva, C.,

Riva, G.,

Riva, N.,

Rizza, M. F.,

Rizzi, E.,

Rodilossi, S.,

Rodriguez-Menendez, V.,
S33

S10, S18, S41,

S43, S44

S45, S51, S65

S51

S56

S16, S34

$\mathrm{S} 11, \mathrm{~S} 35$

S26

S20

$\mathrm{S} 25, \mathrm{~S} 31$

$\mathrm{S} 52$

S27

S51

S23

S26

S33

S49

S23

S18, S43

S20

S63

S49, S63

S20

S19

S40

S59

S48

S27

S11

S16

S58

S13, S32, S36, S37

S54

S65

S59

S34

S38

S17, S29

S21

S11

S53

S58

S47

S57, S60

S54

S53

S60

S49

S59

S39, S55 
Rolandi, E., $\quad$ S62

Rolle, G., $\quad$ S52

Romano, G., S54

Roppolo, M., $\quad$ S52

Rossi, G., $\quad$ S65

Rossini, P. M., $\quad$ S11

Rubino, E., $\quad$ S48

Rucco, R., $\quad \mathrm{S} 42$

Ruma, D., $\quad$ S52

Rungratanawanich, W., $\quad$ S37

Russo, L., $\quad$ S37, S56

Russo, M., $\quad$ S40

Saccomano, A., $\quad$ S16

Saccucci, S., S30

Sacheli, L. M., $\quad$ S63, S66

Saetta, G., $\quad$ S62

Saibene, F. L., $\quad$ S47

Sala, A., $\quad$ S18

Sala, B., $\quad$ S57

Sala, G.,

Sala, M.,

Salerno, D.,

Salkovic-Petrisic, M.,

Salmaggi, A.,

Salsone, M.,

Salvadori, N.,

Salvalaio, E.,

Salvato, G.,

Sancarlo, D.,

Sancini, G.,

Sandrock, A.,

Santangelo, R.,

Santini, C.,

Saresella, M.,

Sarica, A.,

Sassella, A.,

Sassos, D.,

Savarese, L.,

Sberna, M.,

Scaccabarozzi, C.,

Scalise, A.,

Scarano, E.,

Scarmeas, N.,

Scarpa, P.,

Scarpini, E.,

Schönknech, P.,

Scioli, S.,

Scocchia, L.,

Scuteri, A.,

Semperboni, S.,

Senerchia, A.,
S34, S55, S57, S60

S33

S34

S38

S65

S19

S40

$\mathrm{S} 23$

S6, S66

$\mathrm{S} 21$

S13, S36

$\mathrm{S} 12$

S25, S31

$\mathrm{S} 55$

S16, S34

S19

S37

S22

S57

S22, S61

S65

S49

S66

S13

S66

S11, S18

$\mathrm{S} 11$

S47

S67

S58

S55

S49
Senesi, B., $\quad$ S20

Serchisu, L., S39

Sesana, S., $\quad$ S39

Sgambato, A., $\quad$ S56

Silani, V., $\quad$ S53

Sinforiani, E., $\quad$ S27

Sironi, V. A., $\quad$ S2

Somalvico, F., S52

Soricelli, A., $\quad$ S11

Sorrentino, G., $\quad$ S42

Sorrentino, P., $\quad$ S42

Sorriso, A., $\quad$ S42

Spaziante, F., $\quad$ S52

Spector, A., $\quad$ S14

Stefanoni, G., S33

Stravalaci, M., $\quad$ S33

Stucchi, N., $\quad$ S67

Taglialatela, G., $\quad$ S32

Tallarita, E., $\quad$ S52

Tamborini, M., $\quad$ S16

Tamburin, S., $\quad$ S26

Tarducci, R., $\quad$ S40

Tassi, L., $\quad$ S66

Terruzzi, A., $\quad$ S65

Terzi, A., $\quad$ S65

Terzuoli, S., $\quad$ S22

Tettamanti, M., $\quad$ S22

Tirelli, P., $\quad$ S24

Toraldo, A., $\quad$ S25

Tornatore, G., S52

Torrisi, J., $\quad$ S27

Tortora, P., S59

Totino, L., $\quad$ S35

Trabattoni, S., $\quad$ S37

Tredici, G., $\quad$ S58

Tremolizzo, L., $\quad$ S4, S23, S32, S33,

S39, S52, S53, S55, S61

Trentin, M., $\quad$ S26

Tresoldi, V., $\quad$ S34

Tsolaki, M., $\quad$ S11

Uberti, D., $\quad$ S19, S37

Ursino, N., $\quad$ S63

Vaccaro, M., $\quad$ S19

Valente, M., $\quad$ S17, S29

Valsecchi, M., $\quad$ S67

Vanoli, E. G., $\quad$ S18

Varriale, P., $\quad$ S42

Vassalli, M., $\quad$ S56

Vasta, R., $\quad$ S19

Vecchio, M. M., $\quad$ S27 
Veneziano, M.,

Verderio, C.,

Verma, A.,

Verniccio, L. S.,

Vianello, G.,

Viganò, B.,

Viglietta, V.,

Visentin, C.,

Vitali, P.,

Weller, R. O.
S20

S33

S3

S27

S26

S51

S12

S59

S17, S29

S3
Williams, L.,

Zanette, G.,

Zani, A.,

Zapparoli, L.,

Zerbi, A.,

Zoia, C. P.,

Zoia, C.,

Zucca, M.,

Zucca, S.,
$\mathrm{S} 12$

S26

S49, S63

S62, S63, S67

S63, S67

S33, S39

S32, S57

S48

S27 


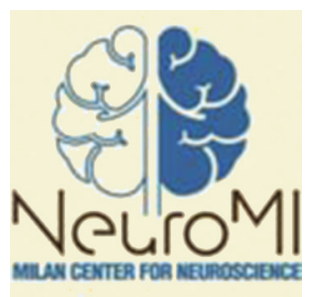

\section{Milan Center for Neuroscience (www.neuromi.it)}

\section{Challenges of modern neuroscience}

Understanding brain function, mechanisms of healthy nervous system development and aging, mechanisms of neurological and psychiatric disorders represents one of the major challenges for humans.

With world-wide aging populations, the burden of neurological diseases is increasing and represents a major cost for society.

Despite the recent technological and scientific advances (high throughput technologies, NMR, PET, discovery of genetic determinants of diseases and of their molecular pathways), disorders such as autism, schizophrenia, Alzheimer, Parkinson, Multiple Sclerosis, Amyotrophic Lateral Sclerosis can be accurately diagnosed at the earliest phases, but still lack effective long-term disease-modifying treatments.

We recently recognized that molecular mechanism leading to these disorders may start even decades before clinical presentation, and that effective treatments should start before brain damage become clinically relevant. For this reason, a major challenge for modern neuroscience is to detect the border between brain function and dysfunction with a multidisciplinary approach: clinical neurology and psychiatry, psychology, neuroimaging, computational science, genetics and biology.

Furthermore, congenital or acquired brain damage most frequently leads to severe impairment of sensorimotor and superior cognitive functions (e.g. language, memory, attention, awareness) that need accurate diagnosis and effective rehabilitation. Hence the compelling needs to further understand the cognitive functions of the normal brain and to develop novel strategies for rehabilitation of braindamaged individuals, following multidisciplinary approaches and up-to-date instrumental monitoring.

Finally, the challenge of modern society and its economic and social determinants, more and more strongly rely upon the contribution of the modern cognitive neuroscience, to understand the core mechanisms regulating interactions among individuals and social behaviour.

\section{NeuroMi Goals}

To promote high-level and multidisciplinary research and education in the field of neuroscience, to uncover mechanisms of nervous system function and dysfunction across the life cycle.

\section{Strategy}

The effort to accomplish this challenging goal must be based on a multidisciplinary team encompassing basic and clinical neuroscientists, and taking advantage of collaborations with institutions at regional, national and international level.

Expertise spans from genetics, molecular biology, cell-, tissue- and in vivo-neuroimaging, psychology, neurology, neuropsychology, neurosurgery, psychiatry, child neuropsychiatry, computational technologies, informatics, statistics, system biology, economy, physics with the use of a large variety of technological platforms.

\section{Research Areas}

Clinical Neurosciences

Molecular and Cellular Neurosciences

Biotechnology and Nanomedicine

Computational and Systems Neurosciences

Neuroimaging and Methodological Research

Cognitive and Behavioral Neurosciences

\section{Research area Clinical Neuroscience}

The research activity of the Clinical Neuroscience Area takes advantage of the presence of different clinical disciplines, such Neurology, Neurosurgery, Neurotology, Maxillo-facial Surgery, Psychiatry, Child Neuropsychiatry, Geriatric Medicine and Neurorehabilitation.

Clinical and translational studies are performed on patients, animal and cellular models of diseases to elucidate the mechanisms involved in the pathophysiology of neurologic and mental diseases at the molecular, cellular and system levels, and to translate these findings into clinical diagnosis, treatment and prevention strategies. Genetic and epidemiological studies take advantage of large cohorts of patients, in collaborations with international networks. 
Main research areas involve mechanisms of neurodegenerative processes, focused on glutamate excitotoxicity, oxidative stress, the amyloid cascade and vascular damage in the brain. These phenomena are studied in patient samples, with the aim to identify molecular markers of disease and to test possible pharmacological interventions. In parallel, neuropsychological and imaging projects are carried out for a better characterization of neurodegenerative disorders (typical and atypical dementias and mild cognitive impairment, Parkinson's disease and amyotrophic lateral sclerosis), and for the development of accurate diagnostic tools (with a special interest for Positron Emission Tomography tracers and MR imaging).

Phase II and III trials are ongoing in patients with Alzheimer's disease and mild cognitive impairment, multiple sclerosis and stroke. A multi-national observational registry on acute stroke recanalization therapies is ongoing since 2005, and a translational research programme of experimental neurology and neurosurgery using rat models of focal cerebral ischemia and intracerebral haemorrhage is being carried out since 2011. International, multicentric clinical trials on chemotherapy-induced peripheral neurotoxicity are coordinated by researchers of this area, but interest is also focused on metabolic, inflammatory and inherited neuropathies.

Other research areas are molecular and genetic and immunological treatment of gliomas, miniinvasive surgical techniques for the treatment of craniofacial and craniovertebral junction malformations and neuroendoscopy for pediatric hydrocephalus and neural tube defects, autism spectrum diseases or chromosomal and genetic abnormalities. Moreover, research interests encompass fine-grained psychopathological description of developmental profile of premature babies, along with the dynamics of couples whose children are affected by psychiatric disorders, and with the clinical endophenotypes and possible biological correlates of anorexia nervosa and borderline personality.

A motion-capture tool is available for studies on treatment of movement dysfunctions (eg. low back pain) and paretic hand. The neuropsychological research spans from the use of Transcranial Direct Current stimulation for the improvement of focal deficits like apraxia and hemianopia, to the use of specific operative strategies like the Constrain Induced Aphasia Therapy.

Specific aspects of psychiatric disorders, such as addiction, social cognition in schizophrenia, correla- tions between personality and mood disorders, or impact of severe mental disorders on family members are investigated.

Geriatric cognitive syndromes such as delirium are widely investigated, in collaboration with national and international research groups.

\section{Research Area Molecular and Cellular Neurosciences}

The research activity of this area involves several aspects of basic neuroscience, with a focus on the translational aspects of the studies in both the central and the peripheral nervous system. Advanced in vitro, ex vivo and in vivo models have been specifically developed to investigate the processes leading to the normal development, maturation and ageing of the nervous system, as well as the detection of pathogenetic mechanisms at the basis of neurological and psychiatric diseases. Multidisciplinarity is an area key word, since basic researchers work in strict collaboration with neurologists, neurosurgeons, psychiatrists and clinical researchers from related fields.

In the field of neurodegeneration, research on oligomeric Abeta and glutamate toxicity, as well as Pin1 isomerase activity are coupled with experimental therapeutic attempts in fibroblasts from Alzheimer's disease patients and neuroblastoma cells. Voltage-gated currents and postsynaptic potentials, functional alterations of the blood-brain barrier (BBB) are also investigated. The role of Parkinson's disease-related toxins, genetic alterations and neuroprotective agents on lysosomal catabolic pathways for alpha-synuclein are also investigated, as well as cholinergic and hypocretinergic regulation of synaptic circuits in the prefrontal cortex. Cerebrotendinous xanthomatosis and Spastic paraplegia type 5 are used as models of neurodegeneration due to defective cholesterol metabolism. Metalloprotease ADAM10 is investigated as a new potential target in the Huntington's disease brain. Prion Protein $(\mathrm{PrPc})$ folding in neuronal membranes and lipid rafts constituents are also established research topics, linked with investigations on air pollution relevance in protein misfolding and neurodegenerative diseases. Recently, the studies in the advanced field of induced pluripotent stem (iPS)-derived neurons from fibroblasts have also been started.

Experimental stroke models in vivo have been developed in order to reproduce transient focal cerebral ischemia and intracerebral hemorrhage, with the final aims to determine the pathogenesis of neuronal 
damage and to test advanced experimental therapies, including collateral therapeutics and selective cerebral spinal fluid hypothermia.

Search for new candidate genes involved in epileptic phenotypes and autism through next generation and microarray techniques and identification, in vitro and in vivo characterization of new mutations in known genes involved in epilepsies are performed by genetists in collaboration with neurologists, neurophysiologists and child neuropsychiatrist. The effects of newly developed peptidic and non peptidic drugs are studied in the in vivo model of pilocarpine-induced epilepsy.

Neuro-oncology is a field of close contact between basic researchers, neurosurgeons, genetists and pathologists, addressing the issue of the pathogenesis and treatment of brain cancer. Innovative treatments based on the use of frontline therapy using synchrotron radiation and photoactivation of suitable drugs (e.g. high z-number compounds) are under investigation in collaboration with the European synchrotron radiation source in Grenoble.

Together with radiotherapy, systemic chemotherapy is one of the cornerstones of cancer treatment, but neurotoxicity is currently the major dose limiting side effect after hematological toxicity. Cellular and animal models of chemotherapy-induced peripheral neurotoxicity have been originally developed and characterized by researchers of this area, and they represent the international "gold-standard" in this fields.

Neuropathic pain, a challenging clinical problem in affected patients, is investigated in vitro and in vivo at the behavioral, pharmacological, pathological and neurophysiological levels and innovative therapeutic approaches are attempted, also in collaboration with leading International pharmacological companies.

The possibility to allow effective neuro-regeneration and immune-modulation using mesenchymal stem cells (MSCs) is tested in well-characterized cellular and animal models. The paracrine actions of MSCs on different cell populations of the central nervous system are investigated in in vitro cellular models. Moreover, the critical aspect of genetic stability of MSCs is investigated through their genomic-epigenomic profiling and non-nervous adult cell transplants are explored to treat neurological complications of systemic diseases (e.g. diabetes).

Multiple sclerosis, and more broadly the field of neuro-inflammatory diseases of the central and peripheral nervous system, is investigated in close collaboration with neurologists and experts in basic immunology at the cellular and animal levels. Integrated chemical-biology approach to the study of Toll-Like Receptor 4 (TLR4) activation and signaling, implication of TLR4 in innate immunity, inflammation and neuro-inflammation, development of small molecule TLR4 modulators as new therapeutics for neuro-inflammatory and neurodegenerative diseases are main topics in this field.

The cellular and molecular mechanisms underlying synaptic formation and function and their roles in the developmental of neuro-behavioural disorders, such as autism and schizophrenia are carried out in collaboration by pharmacologists and adult/ child psychiatrist, while another research line is focused on the genetic study of rare malformative syndromes associated to mental retardation.

Several transduction and intracellular signaling systems are explored in neurons and glial cells, including small G-proteins, Ras and Ral activators, Map kinases, deubiquitinating enzyme USP8, mitochondrial structures and their modulation as well as the use of trophic factors (e.g. NGF, NGF-like molecules) for therapeutic aims is investigated also profiting from system biology methods. Functional and pharmacological studies of ion channels are applied to genetic diseases.

\section{Research Area Biotechnology and Nanomedicine}

The Biotechnology and Nanomedicine Area encompasses competencies in biochemistry, biology, physics, chemistry, material science, physiology, pharmacology, neurology. Translational studies are performed on human specimen, animal and cellular models of disease to elucidate the molecular bases of brain dysfunction involved in the pathophysiology of neurologic diseases and to translate these findings to clinical diagnosis and treatment. Biotechnology is any technological application using biological systems, living organisms or derivatives, to make or modify products or processes. Biotechnology in UNIMIB has its main applications in clinical proteomics, in biosensing and therapy. Nanomedicine, an offspring of biotechnology, is exploiting the use of devices with size in the order of billionths of meters (nanoparticles, nanobiosensors) for therapy and diagnosis of human diseases. Nanomedicine in UNIMIB has its main applications in diagnostics, therapy and tissue regeneration.

Electrophysiology is crucial in the understanding of the electrical functioning of neurons in the central 
nervous system (CNS). Both, the single cell- and the slice-level approaches on samples coming from normal tissue and from diseased brains are central for the analysis of the pathophysiological mechanisms responsible for diseases and for the development of new specific drugs. The availability of 64- and 256-electrode multi-site recording apparatus (MEA) allows to test nanomedicine tools and pharmacological agents on living neuronal networks. This offers a way to study in vitro the effects on neuronal cell health, neurotransmission and neurodegenerative processes.

Proteomics is devoted to assess protein pattern in human samples, with the aim to identify molecular markers of disease and to follow the outcome of pharmacological interventions of neurological diseases. Proteomics unit is accustomed with the main proteomics strategies. Nanomedicine researchers have achieved particularly significant advancements in the applications of nanomedicine for theranostic of the diseases that affect the Central Nervous System (CNS). This approach makes the Athenaeum an outstanding reference Centre in Europe for this particular branch. Relevant results have been achieved thanks to several past and currently running grants devoted to design of nanoparticles (NPs) for treatment and diagnosis of CNS diseases.

\section{Research Area Computational and Systems Neurosciences}

The variety and complexity of data gathered from biomolecular and neuroimaging studies require an appropriate support, both algorithmic and numerical, from the disciplines of computer science, mathematical modeling, and statistical modeling, in order to provide researchers from the biomedical fields with a more extended toolset capable to aid in the identification of particular pathological phenomena; e.g., using novel "pattern recognition" techniques. Modern neurosciences are not limited to the study and description of single altered parameters, but they frame them in the context of different processes and stages, and, in the clinical case, in the context of "disease and disorder models", which are concerned with the progression of several observable phenomena. Such progressions need to be measured and often reconstructed from cross-sectional data; moreover they need to be described by means of controlled vocabularies and ontologies in order to communicate concise, shared, and informative summaries to different researchers and clinical practitio- ners. Many of the algorithmic and numerical underpinnings of such multifarious methods require sophisticated and large-scale computational infrastructure, not to mention the special issues that arise from direct modeling and, above all, simulation of neuronal assemblies and activities. Moreover, there are several novel research directions which combine HW and SW technologies in order to provide neuromorphic computing systems, whose aim can be both applicative and explorative, especially when targeted toward simulation of brain functions.

Within the H2020 program, the area "Information and Communication Technology", alongside the theme "Exascale and Parallel Computing", is foundational to most "Excellent Science" research programs, including those clustered under the Health heading. Among these, the theme "Systems Biology" appears under different guises. Within this framework, the Computational and Systems Neurosciences research area fosters several integration activities of the data generated from the different kinds of studies just mentioned, also as a consequence of the cross-cutting effort on ICT, Parallel Computing, Big Data administration and management, and more traditional algorithmic and computational research.

\section{Research Area Neuroimaging and Methodological Research}

The neuroimaging and methodological research program is an interdisciplinary program that applies imaging, computer sciences and signal analysis techniques to the study of brain functions. The program allows the access to advance technologies and instrumentation located in different institution of the Center. The methodological group is composed by neurologists, psychologists, nuclear physicians, pharmacologist, physicists, computer scientists, statisticians radio-chemists, with expertise in neuroimaging and signal analysis. The program is focused on bringing together clinicians and scientists with the common goal of studying the neurobiology of the brain in normal conditions and diseases at structural, functional and molecular levels using state of the art neuroimaging and signal processing techniques. Imaging and signal processing methodologies may be applied to different fields of neurosciences both at preclinical and clinical levels using both in vitro and in vivo approaches. Main technologies and methodological expertise available for the program are: functional and structural MRI imaging, in vivo and 
in vitro molecular imaging, signal processing and instrumentation development/optimization, computer sciences and statistics.

The Institute of Molecular Physiology and Bioimaging of the National Research Council (IBFMCNR) contributes with research activities aimed at developing methods to support the study, prevention, diagnosis, prognosis and treatment of neurological diseases. These includes: Statistical Parametric Mapping -SPM of PET, SPECT, MRI images, Support Vector Machine - SVM); 2) methods for Quantification of characteristic parameters of neurological diseases (Segmentation techniques of MRI images, for the estimation of volumes, eg. hippocampus in $\mathrm{AD}$; Techniques for correction of physical effects on PET images - partial volume correction); 3) Methods for measuring brain connectivity under health and pathological conditions (Statistical Parametric Mapping -SPM of PET and MRI images for the study of connectivity in several cognitive domains - eg. memory); Methods of integration of biomedical parameters obtained for each subject using a variety of methods: diagnostic and in vivo functional PET, SPECT, CT, MRI imaging, ex vivo molecular imaging (histopathology, proteogenomics), response to therapy; multivariate analysis techniques - eg cluster analysis).

\section{Research Area Cognitive and Behavioral Neurosciences}

Born around a core-group coming from the Milan experimental psychology and neuropsychology schools, founded by Cesare Musatti and Ennio De Renzi respectively, the group has attracted top scientists from the whole of Italy and now counts about 50 members including also developmental psychologists, experimental social psychologists, linguists, all based at the local Psychology Department. The group also includes clinical neuropsychologists of the Clinical Neuroscience Departments and economists interested in cognitive neuroscience. The scientific production of the $\mathrm{CNG}$ fares more than 60.000 citations and more than 560 points of $\mathrm{H}$ index. With this record-track, the CNG has a leading role in Italian cognitive neuroscience.

Aim of this area is the study of the healthy and diseased mind in developing and adult individuals. Intervention areas comprise cognitive psychology in healthy individuals, social psychology and quantitative psychology, neuropsychology in brain-damaged and psychiatric patients for heuristic and rehabilitative purposes, clinical psychology in psychological and psychiatric diseases, neuroeconomics. More specifically, typical areas of enquiry are attention and controlled processes, reasoning, spatial cognition, motor control, language and reading, memory, visual perception and semantics, face processing, social cognition and theory of mind, motivation and emotion. These topics are studied in both normal and abnormal populations along the life cycle. Methods comprise behavioral studies in dedicated labs, as well as advanced instrumental methods for the study of the nervous system activity including neuroimaging (fMRI, PET, EEG mapping and ERP), neurostimulation (tDCS, TMS, EEG/TMS), motion capture (SMART), EMG, GSR, Eye tracking. Fruitful interactions are ongoing with all sectors of the NeuroMi Center, with particular attention to quantitative computational modelling of cognitive processes and improved statistical inference on neurophysiological and imaging data. Innovative interactions are planned with the neurobiology oriented (genetics, pharmacology, neurology and psychiatry. etc.) members of NeuroMI as well as with the Clinical Neurosciences area. 